(C) 1998 International Press

Adv. Theor. Math. Phys. 2 (1998) $1307-1372$

\title{
Heterotic String/F-theory Duality from Mirror Symmetry
}

\author{
${ }^{a}$ P. Berglund, ${ }^{b}$ P. Mayr \\ ${ }^{a}$ Institute for Theoretical Physics, \\ University of California, \\ Santa Barbara, \\ CA 93106, USA \\ berglund@itp.ucsb.edu \\ ${ }^{b}$ Theory Division, \\ CERN, \\ 1211 Geneva 23, \\ Switzerland \\ Peter.Mayr@cern.ch
}

\begin{abstract}
We use local mirror symmetry in type IIA string compactifications on Calabi-Yau $n+1$ folds $X_{n+1}$ to construct vector bundles on (possibly singular) elliptically fibered Calabi-Yau $n$-folds $Z_{n}$. The interpretation of these data as valid classical solutions of the heterotic string compactified on $Z_{n}$ proves $\mathrm{F}$-theory/heterotic duality at the classical level. Toric geometry is used to establish a systematic dictionary that assigns to each given toric $n+1$-fold $X_{n+1}$ a toric $n$ fold $Z_{n}$ together with a specific family of sheaves on it. This allows for a systematic construction of phenomenologically interesting $d=4 N=1$ heterotic vacua, e.g. on deformations of the tangent bundle, with grand unified and $S U(3) \times S U(2)$ gauge groups. As another application we find non-perturbative gauge enhancements of the heterotic string on singular Calabi-Yau manifolds and new non-perturbative dualities relating heterotic compactifications on different manifolds.
\end{abstract}

e-print archive: http://xxx.lanl.gov/abs/hep-th/9811217 


\section{Introduction}

The heterotic string compactified on a Calabi-Yau three-fold $X_{3}$ has been the phenomenologically most promising candidate amongst perturbatively defined string theories for quite some time [1] In particular, compactifications with $(0,2)$ supersymmetry can easily lead to realistic gauge groups [2]. The definition of the theory involves the understanding of a suitable stable vector bundle $V$ on $X_{3}$, which turns out to be a very difficult problem, however.

A new promising approach to the problem is given by the duality to F-theory [3]. The basic duality is between F-theory on elliptically fibered $\mathrm{K} 3$ and the heterotic string on $T^{2}$ in eight dimensions [3, 4]. Lower dimensional dualities are obtained by "fibering the eight-dimensional duality" with the result that $\mathrm{F}$-theory on an elliptically and $\mathrm{K} 3$ fibered $n+1$-dimensional Calabi-Yau manifold $X_{n+1}$ is dual to the heterotic string on an elliptically fibered $n$-dimensional Calabi-Yau $Z_{n}[5]$

Quantum corrections of various kinds on both sides are expected to challenge the usefulness of these dualities for the case of minimal supersymmetry. However, to not put the cart before the horse, the first task should be to determine dual compactifications in a classical sense, by specifying to each Calabi-Yau manifold $X_{n+1}$ for the F-theory compactification the dual manifold $Z_{n}$ together with an appropriate bundle ${ }^{1} V$ on it. A big step in this direction has been done in the mathematical analysis of $[7,8,9,10]$ where the moduli space of holomorphic $H$ bundles on elliptically fibered manifolds has been determined.

In this paper we take a rather different route to F-theory/heterotic duality. We define vector bundles on general elliptically fibered Calabi-Yau $n$-folds purely in a type IIA language. Specifically, the type IIA theory compactified on an elliptically and K3 fibered Calabi-Yau manifold $X_{n+1}$ describes holomorphic $H$ bundles on an elliptically fibered Calabi-Yau manifold $Z_{n}$ on general grounds, without any reference to a heterotic dual. As the starting point consider a type IIA compactification on $\mathrm{K} 3 \times T^{2}$ where the $\mathrm{K} 3$ is elliptically fibered and has a singularity of type $H$. Part of the moduli space $\mathcal{M}_{I I A}$ is identified with the moduli space $\mathcal{M}_{T^{2}}$ of Wilson lines on $T^{2}$. The $R$-symmetry of the $N=4$ supersymmetry of this compactification provides identifications in $\mathcal{M}_{I I A}$ which in particular relate Kähler deformations of the singularity $H$ in the elliptic fibration of the $\mathrm{K} 3$ to $\mathcal{M}_{T^{2}}$ [11]. The correspondence can be made precise by considering a certain local limit. Application of local mirror symmetry maps this description of $\mathcal{M}_{T^{2}}$ in terms of

\footnotetext{
${ }^{1}$ Or more generally, reflexive or coherent sheaves [6]
} 
Kähler moduli to a description of $\mathcal{M}_{T^{2}}$ in terms of complex deformations of a local mirror geometry ${ }^{2} \mathcal{W}_{2}$.In particular, $\mathcal{W}_{2}$ gives a concrete description of the elliptic curve $T^{2}$ and a flat $H$ bundle on it, where $H$ is the type of the original singularity we started with. Combining this construction with an adiabatic argument as in [12], we can use an equivalent limit of a type IIA compactification on a Calabi-Yau $n+1$-fold $X_{n+1}$ rather than K3, to describe deformations of a weighted projective bundle $\tilde{\mathcal{W}} \rightarrow Z_{n}$ on the elliptically fibered manifold $Z_{n}$. These data define a family of vector bundles $V$ on $Z_{n}$ [8]. Taking the small fiber limit of $X_{n+1}$, which does not interfer with the limit extracting the submoduli space of the holomorphic bundle on $Z_{n}$, we arrive at a similar conclusion for the F-theory vacua in two dimensions higher. F-theory/heterotic duality reduces to the mere statement that we can now interprete the stable $H$ bundle on $Z_{n}$ as a classical solution of the heterotic string.

Our approach improves in various aspects the previous understanding and use of heterotic/F-theory duality. First note that rather than comparing properties of two supposedly dual theories such as the topological data of line bundles in [8], we derive F-theory/heterotic duality from known, classical physics of the type IIA compactification. On the practical side, the method allows us to engineer a bundle with any given structure group $H$ on any elliptically fibered Calabi-Yau manifold $Z_{n}$ with no restriction on the smoothness of the elliptic fibration. Using the powerful concept of toric geometry we can give a systematic construction of how to build the Calabi-Yau manifold $X_{n+1}$ from a few elementary building blocks. Subtleties arising from singularities in the elliptic fibrations are taken care of by the toric framework.

That a purely classical type IIA framework can be used to provide a geometric construction of a supersymmetric quantum theory has a wellknown prehistory. In the geometric realization of the result of Seiberg and Witten [13] on $N=2$ supersymmetric Super-Yang-Mills (SYM) theory, the moduli space is described in terms of periods of a Riemann surface $\Sigma$. The a priori surprising appearance of the complex geometry $\Sigma$ is explained by the fact that it appears as the mirror geometry of a type II Calabi-Yau three-fold compactification $[14,15,16,11]$. Once this relation is recognized, it is much simpler and much more general to obtain the exact answer for SYM theories in the type II setup, with the result that for general gauge group $H$, the Seiberg-Witten geometry is a Calabi-Yau threefold rather than a curve [11], as anticipated by the string point of view.In the present case, the mathematical analysis of [8] leads to a formulation of the moduli

\footnotetext{
${ }^{2}$ Here and in the following a subscript denotes the complex dimension of a geometry.
} 
space of $H$ bundles on an elliptically fibered Calabi-Yau $Z_{n}$ in terms of deformations of seemingly unrelated geometries of various dimensions for various $H$ [8]. Again these geometries are identified in the present paper directly with a physical type IIA compactification. These geometries are well-understood and together with toric geometry provide an easy kit to construct any given combination of a family of bundles and a manifold. We will formulate our construction in terms of Calabi-Yau manifolds represented as toric hypersurfaces, which also in many other respects is the most useful and most general representation of these manifolds.

The organization of this paper is as follows. In sect. 2 we explain the basic set up, in particular the local mirror map, and give an outline of the general recipe for lower-dimensional theories. In sect. 3 we define the toric geometries and their mirrors for the eight-dimensional case and describe how the polyhedron $\Delta_{X_{n}+1}^{*}$ associated to the toric manifold $X_{n+1}$ in the description of toric geometry, encodes the toric manifold $Z_{n}$ in terms of a projection. In sect. 4 we complete the eight-dimensional dictionary between toric $\mathrm{K} 3$ manifolds and $H$ bundles on an elliptic curve. In sect.5 we illustrate the six-dimensional case, giving a dictionary between local degeneration of Calabi-Yau threefolds $X_{3}$ on the one side and a family of vector bundles with structure group $H$ on a K3 manifold $Z_{2}$. We find that a singular heterotic manifold can lead to non-perturbative symmetry enhancement for appropriate choice of gauge bundle. Many of these theories turn out to have a non-perturbative dual with a gauge bundle with different structure group on a different $\mathrm{K} 3$ manifold. In sect.6 we describe vector bundles on Calabi-Yau three-folds which may serve as a classical vacuum of a four-dimensional $N=1$ heterotic string. We discuss compactification on the tangent bundle generally in $10-2 n$ dimensions and find similar non-perturbative equivalences as those in the six dimensions. Some phenomenological interesting configurations with gauge groups $E_{8}, E_{7}, E_{6}, S O(10), S U(5)$ and $S U(3) \times S U(2)$ are considered in sect.7. We end with our conclusions in sect.8.

\section{Holomorphic bundles on elliptically fibered Calabi-Yau's from mirror symmetry}

For the class of heterotic vacua with F-theory duals we can restrict to the case of vector bundles on elliptically fibered manifolds. For smooth fibrations, the vector bundle $V$ on $Z_{n}$ can then be thought of in a fiberwise way as the situation where the data of a flat $H$ bundle on the elliptic curve $E$ varies over the points on the base. This case has been analyzed mathematically in $[8,10]$. We then analyze the type IIA aspect, stressing the natural appearance of the 
mirror manifold, $W_{2}$, and how the moduli space of $H$ bundles is described in terms of complex structure deformations of $W_{2}$. From this we are lead to the local mirror limit and how the construction generalizes to lower dimensional theories.

\section{The heterotic perspective}

Let us recall the basic construction of ref.[8]. In [7] it was shown that the moduli space $\mathcal{M}_{E}$ of a holomorphic $H$ bundle on the elliptic curve $E$ is a weighted projective space $W=\mathbf{W P}_{s_{0}, \ldots, s_{r}}^{r}$, where $r$ is the rank of $H$ and $s_{i}$ are the Dynkin numbers of the Dynkin diagram of the twisted Kac-Moody algebra dual to $H$. In [8], the moduli space $\mathcal{M}_{Z}$ of $H$ bundles on elliptically fibered manifolds $Z$ (not necessarily Calabi-Yau) is described in terms of families of elliptic curves $E_{b}$ together with a bundle $V_{b}$ on $E_{b}$ varying over the base $B$ of the elliptic fibration $\pi: Z \rightarrow B$. Here $b$ parametrizes the base $B$. Part of the data of the bundle $V$ on $Z$ is described by a bundle $\tilde{\mathcal{W}}$ of weighted projective spaces over $B$. Restriction to a point $b \in B$ gives a weighted projective space $W_{b}$ as above, together with an elliptic curve $E_{b}$. The bundle $\tilde{\mathcal{W}}$ is given by a projectivization of the bundle $\Omega$

$$
\Omega=\mathcal{O} \oplus\left(\oplus_{i=1}^{r} \mathcal{L}^{-d_{i}}\right),
$$

where $\mathcal{L}^{-1}$ is the normal bundle of $B$ and the $d_{i}$ are the degrees of the independent Casimir operators of $H$.

For example, in the case $G=S U(n)$, the zero of a section of $\tilde{\mathcal{W}}$ determines a "spectral cover" $C \subset Z$, a codimension one submanifold in $Z$. $C$ intersects an elliptic fiber $E_{b}$ at $n$ points which define a holomorphic $S U(n)$ bundle by identifying $E_{b}$ with its Jacobian.

The information provided by a section of $\tilde{\mathcal{W}}$ fixes only the part of the data of $V$ which determines the restriction $\left.V\right|_{E_{b}}$ to fibers of $\pi: Z \rightarrow B$. The information about the non-trivial twisting is contained in a line bundle $\mathcal{S}$ on $C$ [8]. If $C$ is non-simply connected, the definition of $\mathcal{S}$ requires ${ }^{3}$ the specification of holonomies classified by the Jacobian of $C$. In the F-theory compactification on $X$ this information corresponds to a point in the torus $H^{3}(X, \mathbf{R}) / H^{3}(X, \mathbf{Z})$. The moduli space $\mathcal{M}_{Z}$ of the $H$ bundles on $Z$ is thus fibered over the basis $\mathcal{Y}$, the space of sections of $\tilde{\mathcal{W}}$.

In the duality between the heterotic string on $Z$ and $\mathrm{F}$-theory on $X$, the geometric data of $X$ fix only the bundle $\tilde{\mathcal{W}}$. This is clear upon further com-

\footnotetext{
${ }^{3}$ Additional data related to singularities in the fiber product $Z \times{ }_{B} Z$ are required if the complex dimension of $B$ is larger than one. See[64] for a partial identification of these data and refs $[8,10,65]$ for comments on the data related to additional singularities in the construction.
} 
pactification on a two torus, where the torus $H^{3}(X, \mathbf{R}) / H^{3}(X, \mathbf{Z})$ gives rise to $\mathrm{RR}$ fields of the dual type IIA compactification, which are non-geometric.

\section{The type IIA perspective}

The moduli space $\mathcal{M}_{E}$ of flat $H$ bundles on an elliptic curve $E$ appears in a different context in the type IIA compactification on $K 3 \times T^{2}$, corresponding to the Wilson lines on $T^{2}$. As observed in [11], this implies that complex deformations of K3, Kähler deformations of $\mathrm{K} 3$ and Wilson lines on the elliptic curve $E$ can lead to equivalent moduli spaces. Specifically, the three different kinds of deformations can be identified with the three adjoint scalar fields in the $N=4$ vector multiplet in four dimensions. Since there are elements of the $S O(6)$ R-symmetry which rotate the three deformations into each other, one can infer an equivalence of moduli spaces under certain conditions. First note that if we choose a specific algebraic representation $M_{2}$ for a $\mathrm{K} 3$, the Kähler deformations of $M_{2}$ are equivalent to complex deformations of the mirror manifold $W_{2}$, so we may have to switch representations of $\mathrm{K} 3$, when applying R-symmetry transformations. Similarly, the moduli space of $H$ Wilson lines on $T^{2}$ will appear in a type IIA compactification on a $\mathrm{K} 3 Z_{2}$ with an $H$ singularity. The generic theory is mapped by the R-symmetry to a type IIA compactification on a smooth $\mathrm{K} 3, M_{2}$ or $W_{2}$, in which an $H$ singularity is deformed in either Kähler or complex structure.

The moduli space $\mathcal{M}_{E}$ of $H$ Wilson lines on $T^{2}$ contains the geometric deformations of $T^{2}$. In order that these moduli can be equivalent to Kähler deformations of $M_{2}$, the latter has to be elliptically fibered, with the two classes of the fiber and the base related to the moduli of $T^{2}$. Similarly, to have an equivalent representation in terms of complex deformations of a $\mathrm{K} 3$, $W_{2}$, we have to require the mirror $M_{2}$ to be elliptically fibered.

Moreover, in the full string moduli space these deformations are intertwined with each other, so we have to consider a special boundary in moduli space where the deformations are independent. For Kähler deformations of a K3, $M_{2}$, this requires that we restrict to a region in moduli space where the local deformations of a single $H$ singularity decouple from the rest of the global geometry. The corresponding limit in the mirror manifold that gives a representation of $\mathcal{M}_{E}$ as complex deformations is given by translating the previous local limit in Kähler moduli to a limit in complex moduli. It is defined by the action of mirror symmetry on the moduli spaces of $M_{2}$ and $W_{2}$. In the following this limit in $W_{2}$ will be denoted the local mirror limit and the associated local geometry $\mathcal{W}_{2}$.

After having understood the relation between the moduli space of elliptically fibered $\mathrm{K} 3$ and flat bundles on $E$, we can discard the $T^{2}$ in the above 
discussion and consider the six-dimensional type IIA compactification on the $\mathrm{K} 3, M_{2}$. If both $M_{2}$ and $W_{2}$ are elliptically fibered, the complex and Kähler deformations of $M_{2}$ will describe two vector bundles $V, V^{\prime}$ with structure groups $H, H^{\prime}$ on (different) tori $E, E^{\prime}$, respectively. Because of the elliptic fibration we can moreover consider the eight-dimensional small fiber limit of F-theory. The local mirror limit in complex structure now describes $H$ Wilson lines on $T^{2}$.

Our strategy to describe an $H$ bundle on an elliptic curve $E$ is now as follows: we start with an elliptically fibered $\mathrm{K} 3$ manifold $M_{2}(H)$ with a singularity of type $H$ in the elliptic fibration. Applying mirror symmetry to $M_{2}(H)$ we obtain a mirror $\mathrm{K} 3, W_{2}(H)$, with the roles of complex and Kähler deformations exchanged. In the local mirror limit we obtain a complex geometry $\mathcal{W}_{2}$ whose deformations describe the $H$ bundle on $E_{H}$. Fibering the local geometry $\mathcal{W}_{2}$ over a complex base manifold $B$ we obtain an $H$ bundle on the elliptically fibered manifold $Z \rightarrow B$ with fibers $E_{b}, b \in B$.

\section{Type IIA/F-theory/heterotic duality}

Up to now we have obtained the moduli space of $H$ bundles on $Z$ as complex deformations of $\mathcal{W}_{2}$. In addition we have Kähler deformations describing another flat bundle on $T^{2}$.This is also the classical moduli space of the heterotic string on $T^{2} \times T^{2}$ with a vector bundle that splits over the two $T^{2}$ factors. In the small fiber limit this is reduced to the heterotic string on $T^{2}$. The statement that the full moduli spaces are equivalent is the conjectured duality between the heterotic string and the corresponding type IIA/F-theory. In six dimensions, we can interpret the complex and Kähler deformations of the elliptically fibered manifold $W_{2}$ as two sets of Wilson lines $W_{I}$ and $W_{I I}$ with structure groups $H_{I}$ and $H_{I I}$ on a $T_{I}^{2} \times T_{I I}^{2}$ compactification of the heterotic string, respectively. Specifically, the $\mathrm{K} 3 W_{2}$ provides a Kähler resolution of an $H_{I}$ singularity, whereas the complex deformations encode the resolution of an $H_{I I}$ singularity. The decompactification limit of $T_{I}^{2}$ switches off the Wilson lines in $H_{I}$ and restores an $H_{I}$ gauge symmetry. It corresponds to the small fiber limit of $W_{2}$ which blows down the Kähler resolution of the $H_{I}$ singularity in the elliptic fibration. Specifically, the Kähler resolution replaces the singular elliptic fiber by a collection of $\operatorname{rk}(G)+1$ two-spheres which intersect according to the affine Dynkin diagram of $H$. The classes of the blow-up spheres $C_{i}$ are related to the class $E$ of a generic fiber over a generic point by

$$
E=\sum_{i} s_{i} C_{i}
$$

with $s_{i}$ the (positive) Dynkin indices. Thus blowing down the generic fiber blows down the spheres of the $H_{I}$ singularity leading to a gauge symmetry 
enhancement in the type IIA theory.

\subsection{The local mirror limit}

As mentioned before, the classical limit of the moduli space $\mathcal{M}_{E}(H)$ of $H$ bundles on $E$, as represented by a Kähler deformation of an $H$ singularity in an elliptic fibration of a K3, $M_{2}$, corresponds to a limit where we consider only the local deformations of the $H$ singularity and switch off the coupling to the global geometry.

However, we are really interested in taking the same limit for the complex deformations of the mirror $W_{2}(H)$. To be specific consider the case of $\mathrm{K} 3$ manifolds dual to the $E_{8} \times E_{8}$ string. In this case the K3 manifold $M_{2}(H)$ has generically two singularities at the points $z=0$ and $z=\infty$ corresponding to the eight-dimensional gauge group $H=H_{1} \times H_{2}$ in the two $E_{8}$ factors. We represent $M_{2}(H)$ and $W_{2}(H)$ as hypersurfaces in a toric variety. The toric construction will be described in detail in the next section. For the present discussion it is sufficient to know that the mirror manifold $W_{2}(H)$ is described as a hypersurface, given as the zero locus of a polynomial $p_{W_{2}(H)}$ in the toric embedding space. We assert that for the class of K3's dual to $E_{8} \times E_{8}$ string, the polynomial $p_{W_{2}(H)}$ takes the general form

$$
\begin{aligned}
p_{W_{2}(H)} & =p_{0}+p_{+}+p_{-}, \\
p_{0} & =y^{2}+x^{3}+\tilde{z}^{6}+\mu x y \tilde{z} \\
p_{ \pm} & =\sum_{i=1}^{k} v^{ \pm i} p_{ \pm}^{i}
\end{aligned}
$$

where $y, x, \tilde{z}, v$ are specially chosen coordinates on the embedding space; in particular $v$ is a coordinate on the base $\mathbf{P}^{1}$. Moreover $p_{ \pm}^{i}$ are polynomials in $y, x, \tilde{z}$ of homogeneous degree with respect to the scaling action $(y, x, \tilde{z}) \rightarrow$ $\left(\lambda^{3} y, \lambda^{2} x, \lambda \tilde{z}\right)$. Moreover $\mu$ is a complex parameter related to the complex structure of the elliptic curve $\hat{E}_{H}: p_{0}=0$.

The fact that $p_{W_{2}(H)}$ takes the above form is the way in which the complex geometry $\mathcal{W}_{2}(H)$ encodes the information that there is a singularity in each of the two $E_{8}$ factors at the two points $z=0$ and $z=\infty$, respectively. Note that in addition to taking the large base limit in the original K3 $M_{2}(H)$ we have also to make a choice of which of the two points $z=0$ or $z=\infty$ we want to concentrate on. We claim that the local mirror corresponds to take a limit in the complex parameters such that

$$
p_{-}^{i} \rightarrow \epsilon^{i} p_{-}^{i},
$$


with $\epsilon \rightarrow 0$. The local mirror geometry $\mathcal{W}$ is then given by

$$
p_{\mathcal{W}}=p_{0}+p_{+}=0 .
$$

The complex deformation of the geometry $p_{\mathcal{W}}=0$ describes the moduli space of a flat bundle $V$ compactified on the elliptic curve $E_{H}$ defined by the hypersurface $v=0, \hat{E}_{H}: p_{0}=0$. More precisely $\hat{E}_{H}$ is the dual of the torus $E_{H}$; in other words, we can think of $\hat{E}_{H}$ as the Jacobian of $E_{H}$, which shares the complex structure with $E_{H}$. The polynomial $p_{+}$contains the information about a bundle $V_{+}$on $E_{H}$. E.g. for $H=S U(N), N$ even, we will have

$$
p_{+}=v\left(\tilde{z}^{N}+\tilde{z}^{N-2} x+\tilde{z}^{N-3} y+\ldots+x^{N / 2}\right) .
$$

As explained in more detail in a moment, we can integrate out $v$ and obtain a geometry defined by the two equations $p_{0}=0 \cap p_{+}=0$. This intersection gives $N$ points on $\hat{E}_{H}$, which are interpreted as the values of the Wilson lines in the Cartan algebra of $S U(N)$. These data specify uniquely the $S U(N)$ bundle $V_{+}[8]$

Via duality, we will interpret this bundle as a bundle in the first $E_{8}$ factor of the heterotic string. Since the original K3 had two singularities, the limit 2.4 must already include a choice of neighborhood. To describe the neighbourhood of the second singularity, we simply rescale the variable $v \rightarrow v \epsilon$ with the result that now the perturbations in $p_{+}^{i}$ scale as $\epsilon^{i}$ while those in $p_{-}^{i}$ are constant. The corresponding bundle $V_{-}$can be interpreted as the bundle in the second $E_{8}$ factor of the dual heterotic string.

The fact that the limit 2.4 is indeed the action on the complex structure moduli obtained from action of mirror symmetry on the local limit in the Kähler moduli space can be shown by a straightforward analysis of the Kähler cone of $M_{2}(H)$ and the mirror map between the Kähler moduli space of $M_{2}(H)$ and the complex moduli of $W_{2}(H)$. This is described in Appendix A using the toric formulation introduced in the next section.

\subsection{Lower dimensional theories}

The above construction will be generalized to lower-dimensional dual pairs of F-theory on Calabi-Yau $W_{n+1}$ and the heterotic string on Calabi-Yau $Z_{n}$ by an application of the adiabatic argument [12] The geometry $W_{2}(H)$ describes an $H$ bundle over $E_{H}$ in the local limit. To obtain the description of an $H$ bundle over an elliptically fibered Calabi-Yau $Z_{n}$ we can fiber the geometry $W_{2}(H)$ over an $n-1$ dimensional base $B_{n-1}$ to obtain a Calabi-Yau $W_{n+1}$. 
In the local limit we now get an $n$ dimensional geometry $\mathcal{W}$ defined as in 2.3 , but with the polynomials $p_{ \pm}$being functions of the coordinates of the base $B_{n-1}$ (or rather sections of line bundles on $B_{n-1}$ ). Similarly the bundle is now defined on the projection to $p_{0}=0$, which gives an $n$ dimensional Calabi-Yau $\hat{Z}_{n}$. We can identify $\hat{Z}_{n}$ with the dual ${ }^{4} \hat{Z}_{H}$ of a dual heterotic compactification manifold $Z_{H}$. We will return to the higher dimensional case later. For now we note that the local limit is taken only in the fiber $W_{2}(H)$ (we choose to concentrate on the point with the singularity in the K3 fiber), but we retain the global structure of the elliptic fibration over the base $B_{n-1}$.

\section{Toric Construction}

\subsection{General remarks}

In the toric framework, a Calabi-Yau manifold $M_{n}$ is described by an $n+1$ dimensional polyhedron $\Delta$. The vertices $\nu_{k}$ of $\Delta$ lying on faces of codimension larger than one correspond to divisors $x_{k}=0$ in $M_{n}$. Here $x_{k}$ is a coordinate on the toric ambient space associated to $\nu_{k}$. If $n=2$, that is $M_{2}$ is a K3 manifold, then a divisor is simply a holomorphic curve. On the other hand, the Kähler resolution of a local $H$ singularity gives also rise to a collection of holomorphic spheres, intersecting according to the Dynkin diagram of $H$. Therefore the resolution of $H$ corresponds to a set of vertices $\nu_{k}$ in the polyhedron $\Delta$ of $M_{2}(H)$. It is in this way that the construction of the K3 $M_{2}(H)$ and its mirror $W_{2}(H)$ can be phrased entirely in terms of the polyhedra $\Delta$ and $\Delta^{*}$. For $n>2$, the divisors $x_{k}=0$ are no longer curves, but are dual to curves in $X_{n}{ }^{5}$

In general the mirror manifold $W_{n}$, associated to $M_{n}$, can be obtained using Batyrev's construction in terms of of the dual polyhedron $\Delta^{\star}[18]$.The manifolds $M_{n}$ and $W_{n}$ are then defined by the zero of a polynomial as:

$$
p\left(M_{n}\right)=p_{\Delta}=\sum_{j} a_{j} \prod_{i} x_{i}^{\left\langle\nu_{i}, \nu_{j}^{\star}\right\rangle+1}
$$

\footnotetext{
${ }^{4}$ With duality understood as the replacement of the elliptic fiber $E_{H}$ by the dual $\hat{E}_{H}$ parametrizing the Jacobian of $E_{H}$. For simplification we will drop the hat on $Z_{n}$ in the following.

${ }^{5}$ For more details the reader is refered to the reviews and discussions of toric geometry in the physics literature[17]
} 


$$
p\left(W_{n}\right)=p_{\Delta}^{\star}=\sum_{i} b_{i} \prod_{j} x_{j}^{\left\langle\nu_{i}, \nu_{j}^{\star}\right\rangle+1}
$$

where the sum (product) runs over all vertices $\nu_{i}\left(\nu_{j}^{\star}\right)$ of $\Delta\left(\Delta^{*}\right)$ which are on faces of codimension higher than one. The parameters $a_{j}, b_{i}$ determine the complex structure.

If $N$ is the number of (relevant) vertices $\nu_{i}$ of $\Delta$, there will be $N-n-1$ non-trivial relations $\sum_{i} l_{i}^{(r)} \nu_{i}=0$ defined by the vectors $l^{(r)}$. From 3.1 it follows that the equation $p_{\Delta}$ is invariant under rescalings $x_{i} \rightarrow x_{i} \mu^{l_{i}^{(r)}}$. Using these rescalings we can set $N-n-1$ coordinates to one in the appropriate patch and compactified on the remaining equation in $n+1$ variables defines a patch of the Calabi-Yau manifold $M_{n}$ of dimension $n$. The number of such rescalings is precisely the hodge number $h^{1,1}\left(M_{n}\right)^{6}$. This is of course a consequence of the fact that a vertex $\nu_{i}$ in $\Delta$ corresponds to a divisor in $M_{n}$ which in turn defines a $(1,1)$ form $K_{r}$ on $M_{n}$. The same is true for $W_{n}$ with the roles of $\Delta$ and $\Delta^{\star}$ interchanged.

From 3.1 one can see that the relations $l^{(r)}$ translate to relations

$$
\prod_{i} y_{i}^{l_{i}^{(r)}}=1
$$

between the monomials $y_{i}=\prod_{j} x_{j}^{\left\langle\nu_{i}, \nu_{j}^{\star}\right\rangle+1}$ of $p_{\Delta}^{\star}$. In other words, given $p_{\Delta}$ and its scaling relations we can construct $p_{\Delta}^{\star}$ by solving for these relations without having a dual polyhedron. In particular we can take the vertices associated to the resolution of an elliptic singularity as in Table 1 and get an equation for the mirror geometry, which describe a flat $H$ bundle over an elliptic curve [11], for the reasons explained above.

If these vertices are part of a polyhedron $\Delta$ of a compact Calabi-Yau manifold, we can alternatively use Batyrev's construction 3.1 to get the global mirror geometry. This global information contains in particular a complete basis of divisors in $W_{n}$ and the associated rescalings $l^{(r) *}$. The polynomial $p_{\Delta}^{\star}$ obtained in this way agrees with the one obtained from the local description in a certain patch with some of the $x_{i}$ set to one.

\subsection{Mirror geometries for elliptically fibered K3}

We proceed with a construction of $W_{2}(H)$ in terms of toric polyhedra and the derivation of the precise form of 2.5 for various $H$.

\footnotetext{
${ }^{6}$ This assumes that all the Kähler deformations are toric.
} 
The toric resolution of singularities in an elliptic fibration has been studied in $[19,20,11]$ For the case $n=2$, we use the definitions

$$
\begin{aligned}
e_{2} & =(0,-1,0), e_{3}=(0,0,-1), \\
f_{1} & =(0,2,3), f_{2}=(0,1,2), f_{3}=(0,1,1) \\
v_{0} & =(-1,2,3), v_{k}=\left(-1,2-\left[\frac{k+1}{2}\right], 2-\left[\frac{k}{2}\right]\right), \\
w_{k} & =(-2,3-k, 4-k), k>0, \\
s_{1} & =(-2,1,1), s_{2}=(-3,1,1), \quad t_{1}=(-3,1,2), t_{2}=(-4,1,2), \\
u_{1} & =(-3,2,3), u_{2}=(-4,2,3), u_{3}=(-5,2,3), u_{4}=(-6,2,3) .
\end{aligned}
$$

Moreover a tilde will denote a reversal of the sign of the first entry, e.g. $\tilde{v}_{0}=(1,2,3)$. The toric data for an $H$ singularity in the elliptic fibration over a plane are $[19]$ :

\begin{tabular}{|l|l|}
\hline$H$ & $\left\{\nu_{i}\right\}$ \\
\hline$S U(N)$ & $v_{1}, \ldots, v_{N-1}$ \\
$S O(7)$ & $v_{2}, v_{3}, w_{1}$ \\
$S O(2 N+5)$ & $v_{2}, v_{N+1}, w_{1}, \ldots, w_{N}$ \\
$S p(N)$ & $v_{1}, v_{3}, \ldots, v_{2 N-1}$ \\
$S O(2 N+6)$ & $v_{2}, v_{N+1}, v_{N+2}, w_{1}, \ldots, w_{N}$ \\
$G_{2}$ & $v_{2}, w_{1}$ \\
$F_{4}$ & $u_{1}, v_{3}, w_{1}, w_{2}$ \\
$E_{6}$ & $s_{1}, u_{1}, v_{3}, v_{4}, w_{1}, w_{2}$ \\
$E_{7}$ & $s_{1}, t_{1}, u_{1}, u_{2}, v_{4}, w_{1}, w_{3}$ \\
$E_{8}$ & $s_{2}, t_{2}, u_{1}, \ldots, u_{4}, w_{1}, w_{3}$ \\
\hline
\end{tabular}

Table 1:Vertices $\left\{\nu_{i}\right\}$ of the toric polyhedron $\Delta_{\text {local }}=$ convex hull $\left\{e_{2}, e_{3}, v_{0}, \nu_{i}\right\}$ for the resolution of an $H$ singularity in the elliptic fibration over the plane..

Solutions of the relations 3.2 for many $H$ has been given in [11] and will be extended in the next section to all Lie groups. The generic structure is as follows. Firstly we associate to the vertices $e_{i}, f_{1}$ of the elliptic fiber the monomials:

$$
e_{2} \leftrightarrow x^{3}, e_{3} \leftrightarrow y^{2}, f_{1} \leftrightarrow \tilde{z}^{6} .
$$

Moreover we associate to a vertex $\nu_{i} \in \Delta_{\text {local }}$ with first entry $\nu_{i, 1}$ a monomial

$$
v^{-\nu_{i, 1}} f(y, x, \tilde{z}),
$$

such that $f(y, x, \tilde{z})$ is a polynomial which solves the obvious linear relations between the vertices. Note that $(y, x, \tilde{z})$ will appear as homogeneous coordinates of the elliptic curve $\hat{E}_{H}: p_{0}=0$ while $v$ defines a grading of the bundle on $\hat{E}_{H}$. 
Note that the (negative of) first entry of the vertices in (3.4) agrees with the Dynkin label of the twisted Kac-Moody algebra associated to a simple Lie group $H$ shown in Fig. 1. Thus the $v$ powers that appear are also in one to one correspondence with the Dynkin labels. Moreover the number of monomials in $p_{+}^{i}$ is equal to the number of Dynkin labels equal to $i$.

\section{Global K3 mirror manifolds}

While we can use the local description above to define any flat $H$ bundle on the elliptic curve $\hat{E}_{H}$, viewing it as a heterotic vacuum will of course imply restrictions on the possible structure groups $H$. The point is that precisely if $H$ can be embedded in a heterotic gauge group then the local geometry corresponding to $\Delta_{\text {local }}$ of Table 1 can be embedded into a global K3 geometry $M_{2}(H)$ corresponding to a larger polyhedron $\Delta$. In particular we can construct a dual polyhedron $\Delta^{\star}$ describing a global geometry $W_{2}$ that contains the local mirror geometry in a patch. This will be very useful when constructing fibrations of the local mirror geometry over an $n-1$ complex dimensional base. If we construct a global K3 manifold $M_{2}$ with only an elliptic singularity of type $H$ above a single point, we obtain the vertices of $\Delta^{\star}$ shown in Table $2 .^{7}$

There is a nice property of mirror symmetry when acting on the elliptically fibered $\mathrm{K} 3$ manifolds in Table 2 and their generalization with two singularities at the two points $z=0$ and $z=\infty$ of the base $\mathbf{P}^{1}$ (corresponding to an elliptic fibration with a $E_{8} \times E_{8}$ structure):

(*) Let $X_{2}\left(H_{1}, H_{2}\right)$ denote the elliptically fibered K3 manifold with singularities of type $H_{1}$ and $H_{2}$ at $z=0$ and $z=\infty$, respectively. Then the mirror of $X_{2}\left(H_{1}, H_{2}\right)$ is a K3 manifold of type $X_{2}\left(G_{H_{1}}, G_{H_{2}}\right)$, with $G_{H_{i}}$ the commutant of $H_{i}$ in $E_{8}$.

This result is not unexpected in view of the interpretation of K3 mirror symmetry in terms of orthogonal lattices [21]. It can be proven by a straightforward application of Batyrev's construction of mirror manifolds in terms of dual polyhedra.

\footnotetext{
${ }^{7}$ We have performed a simple rotation of basis in order to use the definitions 3.3 .
} 


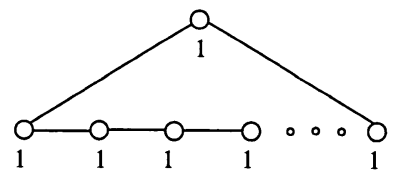

$\mathrm{A}_{\mathrm{N}}$

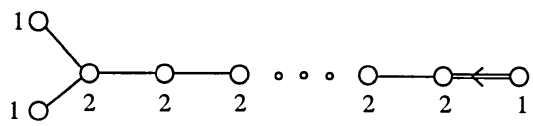

$\mathrm{B}_{\mathrm{N}}$

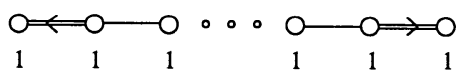

$\mathrm{C}_{\mathrm{N}}$

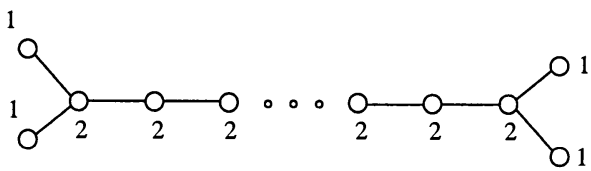

$\mathrm{D}_{\mathrm{N}}$

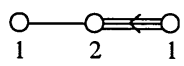

$\mathrm{G}_{2}$

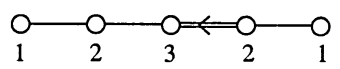

$\mathrm{F}_{4}$<smiles>COOC(OO)OO</smiles>

$\mathrm{E}_{6}$

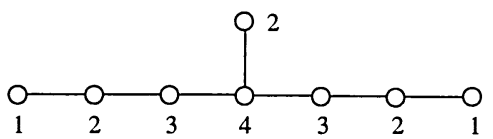

$\mathrm{E}_{7}$

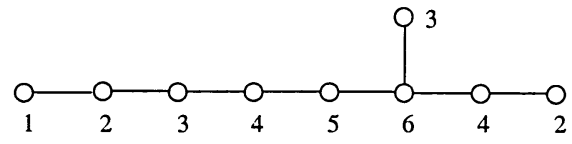

$\mathrm{E}_{8}$

Figure 1: Fig. 1:Dynkin diagrams for the duals of the untwisted Kac-Moody algebras. The integers denote the associated Dynkin labels for the affine root. 


\begin{tabular}{|c|c|c||c|c|c|}
\hline$H$ & $\left\{\nu_{i}^{\star}\right\}$ & $G_{H}$ & $H$ & $\left\{\nu_{i}^{\star}\right\}$ & $G_{H}$ \\
\hline$S U(1)$ & $u_{4}$ & $E_{8}$ & $S O(14)$ & $\tilde{v}_{0}, f_{3}, v_{3}$ & $S U(2)$ \\
$S U(2)$ & $u_{2}, w_{3}$ & $E_{7}$ & $S O(16)$ & $\tilde{w}_{1}, f_{2}, v_{3}$ & $S U(1)$ \\
$S U(3)$ & $s_{1}, u_{1}, v_{4}$ & $E_{6}$ & $S O(5)$ & $w_{1}, w_{3}$ & $S O(11)$ \\
$S U(4)$ & $v_{4}, w_{1}, w_{2}$ & $S O(10)$ & $S O(7)$ & $w_{1}, w_{2}$ & $S O(9)$ \\
$S U(5)$ & $v_{0}, v_{2}, v_{3}, v_{4}$ & $S U(5)$ & $S O(9)$ & $v_{3}, w_{1}$ & $S O(7)$ \\
$S U(6)$ & $f_{1}, v_{1}, v_{3}, v_{4}$ & $S U(2) \times S U(3)$ & $S O(11)$ & $v_{0}, v_{3}$ & $S O(5)$ \\
$S U(7)$ & $\tilde{v}_{0}, f_{3}, v_{3}, v_{4}$ & $S U(2) \times \overline{S U(2)}$ & $S O(13)$ & $f_{1}, v_{3}$ & $S U(2)$ \\
$S U(8)$ & $\tilde{w}_{1}, f_{2}, v_{3}, v_{4}$ & $S U(2)$ & $S O(15)$ & $\tilde{v}_{0}, v_{3}$ & $S U(1)$ \\
$S U(9)$ & $\tilde{u}_{1}, \tilde{v}_{2}, v_{3}, v_{4}$ & $S O(14) \times \overline{S U(2)}$ & $G_{2}$ & $u_{1}$ & $F_{4}$ \\
$S p(3)$ & $f_{1}, w_{3}$ & $G_{2} \times S U(2)$ & $F_{4}$ & $w_{1}$ & $G_{2}$ \\
$S p(4)$ & $\tilde{w}_{1}, w_{3}$ & $S U(2)_{2}$ & $E_{6}$ & $v_{0}, v_{1}, v_{2}$ & $S U(3)$ \\
$S O(10)$ & $v_{0}, v_{2}, v_{3}$ & $S U(4)$ & $E_{7}$ & $v_{0}, v_{1}$ & $S U(2)$ \\
$S O(12)$ & $f_{1}, v_{1}, v_{3}$ & $S U(2) \times S U(2)$ & $E_{8}$ & $v_{0}$ & $S U(1)$ \\
\hline
\end{tabular}

Table 2: Vertices $\left\{\nu_{i}^{\star}\right\}$ associated to the moduli space of $H$ bundles over $E_{H}$ in terms of complex geometries. The toric polyhedron is $\Delta_{H}^{*}=$ convex hull $\left\{\tilde{u}_{4}, e_{2}, e_{3}\right\} \cup$ $\left\{\nu_{i}^{\star}\right\}$. For $H=S U(9) G_{H}$ is the commutant of $H$ in $S O(32)$.

A similar statement applies to fibrations with only a single singularity corresponding to $\mathrm{K} 3$ 's dual to the $S O(32)$ heterotic string. We have indicated the commutants $G$ in Table 2. An over-lined $S U(2)$ denotes a special $S U(2)$ factor which appears as a point in the hyperplane of the elliptic fiber; in this case there is no decomposition as $H \times G$ in terms of maximal subgroups (as e.g. in the breaking $\left.E_{8} \rightarrow S U(7)\right)$.

\subsection{Lower dimensional theories: Heterotic polyhedra from F-theory polyhedra}

To obtain a description of holomorphic bundles on elliptic (Calabi-Yau) manifolds we need a toric description of a fibration of the local mirror geometry $\mathcal{W}_{2}$ above a base manifold $B_{n-1}$. This is a very simple process if $\mathcal{W}_{2}$ can be described in terms of a polyhedron $\Delta^{*}$, which as we noted above is immediate if the structure group $H$ fits into a heterotic gauge group. In this case we have the embedding in a global $\mathrm{K} 3$ geometry $W_{2}$. We will restrict to this simple case, which is also the physically most interesting one in the following. The general case is more involved technically but can be treated very similarly. 
$\operatorname{In}[22]$ it was shown that a toric manifold $X_{n}$ defined by a polynomial as in 3.1 admits a fibration with Calabi-Yau fibers $Y_{k}$, if its polyhedron $\Delta_{X_{n}}$ contains the polyhedron of the fiber $Y_{k}$ as a hypersurface of codimension $n-k$. Thus a fibration of $\mathcal{W}_{2} \subset W_{2}(H)$ over an $n-1$ dimensional base $B_{n-1}$ is described by an $n+2$ dimensional polyhedron $\Delta_{W_{n+1}}^{*}$ that contains $\Delta_{W_{2}}^{\star}$ as a hyperplane. Specifically we can choose coordinates such that the hypersurface $\left\{\nu_{i}^{\star} \in \Delta^{*}: \nu_{i, j}^{\star}=0, j=1, \ldots, n-1\right\}$ contains the vertices $\left(0^{n-1}, \nu_{i}^{\star}\left(W_{2}\right)\right)$, with $\nu_{i}^{\star}\left(W_{2}\right)$ the three-dimensional vertices described in the previous section.

Since we have a well-defined global geometry we can get the defining equation for the mirror manifold directly from 3.1, rather than solving 3.2. After a choice of variables - corresponding to setting some of the toric variables $x_{k}$ to one or equivalently concentrating on the relevant local patch we obtain an expression precisely as in 2.3 , but with the coefficients of the polynomials $p_{ \pm}^{i}$ in $(y, x, \tilde{z})$ being functions of the toric coordinates on $B_{n-1}$. In particular, $Z_{n}: p_{0}=0$ defines an $n$ dimensional Calabi-Yau manifold. The holomorphic $H$ bundle is defined on $Z_{n}$ and we are free to interpret this data as a classical heterotic vacuum.

We have used a limit of the $n+1$ dimensional toric geometries $W_{n+1}$ to describe a heterotic compactification on an $n$ dimensional Calabi-Yau $Z_{n}$ with a prescribed vector bundle. In toric terms, $W_{n+1}$ is given by an $n+2$ dimensional polyhedron $\Delta_{W_{n+1}}^{*}$ and its dual $\Delta_{M_{n+1}}$, while the manifold $Z_{n}$ can be described by an $n+1$ dimensional polyhedron $\Delta_{Z_{n}}^{*}$ and its dual. Let us see how to get $\Delta_{Z_{n}}^{*}$ directly from $\Delta_{W_{n+1}}^{*}$ by an appropriate projection.

First recall that a hypersurface $\mathcal{H}$ in a polyhedron $\Delta$ corresponds to a projection in the dual polyhedron $\Delta^{\star}$. This is evident if we choose coordinates where the $k$ dimensional hypersurface $\mathcal{H}$ is described by vertices $\nu_{i} \in \Delta$ with the first $k$ entries equal to zero. The inner product $\left\langle\nu_{i}, \nu_{j}^{*}\right\rangle$ that determines the monomials in 3.1 does not depend on the first $k$ entries of the vertices $\nu_{j}^{*}$, thus defining a projection in $\Delta^{*}$.

The "heterotic manifold" $Z_{n}$ is defined by $p_{0}=0$ which contains the monomials with zero power of $v$. Recall that in the $\mathrm{K} 3$ case the $v$ power is associated to the first entry $\nu_{i, 1}^{i}$ of a vertex in $\Delta$ and in the above conventions it will be the $n$-th entry of the higher dimensional polyhedron $\Delta_{M_{n+1}}$, the dual polyhedron of $\Delta_{W_{n+1}}^{*}$. Thus the heterotic manifold $Z_{n}$ corresponds to a projection in the $n$-th direction of the polyhedron $\Delta_{W_{n+1}}^{*}$ and its mirror $Z_{n}^{*}$ to a hyperplane $\nu_{i, n}=0$ of the polyhedron $\Delta_{M_{n+1}}$.

$$
f \quad: \quad W_{n+1} \rightarrow Z_{n}
$$




$$
p_{0}=\sum_{i} a_{i} \prod_{j} x_{j}^{\left\langle\nu_{i}^{\prime}, \nu_{j}^{\star}\right\rangle+1} \quad \nu_{i, n}^{\prime}=0
$$

Note that the above implies that the mirror $M_{n+1}$ of the Calabi-Yau $W_{n+1}$ which is dual to the heterotic string on $Z_{n}$ admits a $Z_{n}^{*}$ fibration, $\pi: M_{n+1} \rightarrow$ $\mathbf{P}^{1}$ with fibers $Z_{n}^{*}$, where $Z_{n}^{*}$ is the mirror manifold of $Z_{n}$ !

\section{Local mirror limit of K3 manifolds}

From the toric construction of the elliptic singularities over the plane, see sect.3.2, and solving 3.2 using the variables defined below eq.3.4, we can determine the polynomials $p_{+}^{i}$ appearing in the local limit 2.4. Below we collect the results for the various choices of structure group $H$ of the bundles.

The $H=S U(N)$ cases can be phrased in the general form [11],

$$
p_{+}^{1}=a_{1} \tilde{z}^{N}+a_{2} \tilde{z}^{N-2} x+a_{3} \tilde{z}^{N-3} y+\ldots+\left\{\begin{array}{c}
a_{N} x^{N / 2} \\
a_{N} y x^{\frac{N-3}{2}}
\end{array}\right\},
$$

where the $\left\{a_{i}\right\}$ are coordinates on the moduli space which is isomorphic to $\mathbf{P}^{N-1}$. The geometry $p_{e}+v p_{+}^{1}=0$ describes a two-dimensional complex geometry. Note that we have four coordinates, one equation, and one scaling relation

$$
(y, x, \tilde{z}, v) \sim\left(\lambda^{3} y, \lambda^{2} x, \lambda \tilde{z}, \lambda^{6-N} v\right) .
$$

This is different from the zero dimensional spectral cover description of the moduli space of $S U(n)$ bundles obtained in $[8,10]$. However as far as the complex structure moduli space is concerned, we can integrate out linear variables, that is $v$ in the geometry above, to obtain a zero dimensional geometry

$$
p_{E}=0, \quad p_{+}^{1}=0
$$

the spectral cover. Note that the situation is very similar to what happens in the case of moduli spaces of $N=2 d=4$ SYM theories: the general complex geometry determining the exact solution is a Calabi-Yau three-fold [11] but for the $S U(N)$ case it is natural to integrate out two dimensions $[14,15]$ to get the Riemann surface of [13]. For $S O(2 N+1)$ the local limit can be phrased in the general form

$$
\begin{aligned}
& p_{+}^{1}=b_{1} \tilde{z}^{N-3} y+b_{2} \tilde{z}^{N}+\epsilon b_{3} y x^{\frac{N+1}{2}-2}+(1-\epsilon) b_{3} x^{\frac{N}{2}} \\
& p_{+}^{2}=a_{1} \tilde{z}^{2 N-6}+a_{2} \tilde{z}^{2 N-8} x+\ldots+a_{N-2} x^{N-3}
\end{aligned}
$$


with $\left\{b_{1}, b_{2}, b_{3}, a_{1}, \ldots, a_{N-2}\right\}$ coordinates on the moduli space $\mathbf{W P}_{1,1,1,2, \ldots, 2}^{N}$ of flat $B_{N}$ bundles on elliptic curve $E$. Moreover $\epsilon=(0,1)$ for $N$ even (odd). This expression can be shown to be valid for any $N>4$ by using non-local Calabi-Yau two-fold geometries[23]. The only other case $S O(7)$ is slightly irregular and is obtained from the $S O(9)$ case by discarding the term $a_{2} x$ in $p_{+}^{2}$.

For $S p(N)$ bundles we obtain

$$
p_{+}^{1}=a_{1} \tilde{z}^{2 N}+a_{2} \tilde{z}^{2 N-2} x+a_{3} \tilde{z}^{2 N-4} x^{2}+\ldots+a_{N} x^{N}
$$

with $\left\{a_{i}\right\}$ coordinates on the moduli space $\mathbf{P}^{N}$ of $C_{N}$ bundles. The $S p(N)$ case can be considered as a modding $y \rightarrow-y$ of the $S U(2 N)$ case in 4.1.

$S O(2 N)$ bundles are described by a geometry $\mathcal{W}_{2}\left(D_{N}\right)$ with [11]

$$
\begin{aligned}
& p_{+}^{1}=b_{1} \tilde{z}^{N-3} y+b_{2} \tilde{z}^{N}+c_{1} \tilde{z}^{1-\epsilon} y x^{\frac{N+\epsilon}{2}-2}+c_{2} \tilde{z}^{\epsilon} x^{\frac{N-\epsilon}{2}}, \\
& p_{+}^{2}=a_{1} \tilde{z}^{2 N-6}+a_{2} \tilde{z}^{2 N-8} x+\ldots+a_{N-3} \tilde{z}^{2} x^{N-4} .
\end{aligned}
$$

Here $\left\{b_{1}, b_{2}, c_{1}, c_{2}, a_{1}, \ldots, a_{N-3}\right\}$ are coordinates on the moduli space $\mathbf{W P}_{1,1,1,1,2, \ldots, 2}^{N-1}$ of flat $D_{N-1}$ bundles on elliptic curve $E$, and again $\epsilon=(0,1)$ for $N$ even (odd).

For the exceptional group $G_{2}$ we find

$$
p_{+}^{1}=a_{1} \tilde{z}^{3}+a_{2} y, \quad p_{+}^{2}=b_{1}
$$

with $\left\{a_{1}, a_{2}, b_{1}\right\}$ coordinates on the moduli space $\mathbf{W P}_{1,1,2}^{2}$ of flat $G_{2}$ bundles.

The geometry $\mathcal{W}_{2}\left(F_{4}\right)$ for the exceptional group $F_{4}$ is given by

$$
p_{+}^{1}=a_{1} \tilde{z}^{4}+a_{2} x^{2}, \quad p_{+}^{2}=b_{1} \tilde{z}^{2}+b_{2} x, \quad p_{+}^{3}=c_{1}
$$

where $\left\{a_{1}, a_{2}, b_{1}, b_{2}, c_{1}\right\}$ are coordinates on the moduli space $\mathbf{W} \mathbf{P}_{1,1,2,2,3}^{2}$ of flat $F_{4}$ bundles.

Finally the geometries for the exceptional groups $E_{n}$ can be written as follows ${ }^{8}$. For $E_{6}$ we have

$p_{+}^{1}=a_{1} \tilde{z}^{5}+a_{2} \tilde{z} x^{2}+a_{3} x y, \quad p_{+}^{2}=b_{1} \tilde{z}^{4}+b_{2} \tilde{z} y+b_{3} \tilde{z}^{2} x, \quad p_{+}^{3}=c_{1} \tilde{z}^{3}$,

\footnotetext{
${ }^{8}$ There are several equivalent ways of parametrizing these geometries, see $[8,11]$.
} 
parametrizing a $\mathbf{W P}_{1,1,1,2,2,2,3}^{6}$,

for $E_{7}$

$$
\begin{aligned}
& p_{+}^{1}=a_{1} \tilde{z}^{5}+a_{2} x y, \quad p_{+}^{2}=b_{1} \tilde{z}^{4}+b_{2} x^{2}+b_{3} y \tilde{z} \\
& p_{+}^{3}=c_{1} \tilde{z}^{3}+c_{2} x \tilde{z}, \quad p_{+}^{4}=d_{1} \tilde{z}^{2}
\end{aligned}
$$

defining a moduli space $\mathbf{W} \mathbf{P}_{1,1,2,2,2,3,3,4}^{7}$

and for $E_{8}$

$$
\begin{aligned}
& p_{+}^{1}=a_{1} \tilde{z}^{5}, \quad p_{+}^{2}=b_{1} \tilde{z}^{4}+b_{2} x^{2}, \quad p_{+}^{3}=c_{1} \tilde{z}^{3}+c_{2} y, \\
& p_{+}^{4}=d_{1} \tilde{z}^{2}+d_{2} x, \quad p_{+}^{5}=e_{1} \tilde{z}, \quad p_{+}^{6}=f_{1},
\end{aligned}
$$

giving a moduli space $\mathbf{W P}_{1,2,2,3,3,4,4,5,6}^{8}$. The $E_{n}$ geometries define twodimensional complex del Pezzo surfaces.

The parametrization of the complex geometries as above is ambiguous in the sense that there are additional terms compatible with the scaling symmetries which can be absorbed by variable definitions. E.g. in the polynomial for the elliptic curve $E: y^{2}+x^{3}+\tilde{z}^{6}+y x \tilde{z}=0$ we can eliminate the linear term in $y$ by a shift of $y$ and obtain new monomials $x^{2} \tilde{z}^{2}$ and $x \tilde{z}^{4}$ instead. In the fibrations of the geometries below it may happen that a specific fibration prefers a different parametrization then the one given above. However the monomials always will have the identical scaling properties as the ones given above.

\section{Six-dimensional heterotic $N=1$ vacua}

Let us proceed with six-dimensional dual pairs, that is F-theory on CalabiYau three-fold $W_{3}$ versus the heterotic string on K3. This case has been studied from other various points of view in $[24,25,26]^{9}$.

After a brief discussion of the toric geometry for the elliptic fibration in sect.5.1. we turn to identifying the components of the geometric moduli space in sect.5.2. To demonstrate the method we will describe the construction of smooth bundles on K3 obtained from mirror symmetry in some detail in sect.5.3. In sect.5.4. we discuss gauge backgrounds that lead to

\footnotetext{
${ }^{9}$ In particular the result of local mirror symmetry is technically closely related to the stable degenerations of Calabi-Yau manifolds introduced in [8] and discussed further in [24].
} 
non-perturbative gauge symmetry enhancements. In sect.5.5. we describe the geometric configuration for the tangent bundle. In sect.5.6. we will derive new non-perturbative equivalences in six dimensional heterotic string compactification. The duality involves compactification on quite different $\mathrm{K} 3$ manifolds. In sect. 5.7. we discuss pairs of six dimensional compactifications on mirror Calabi-Yau three-folds that become equivalent after further compactifying to three dimensions. In sect.5.8 we show how the Higgs branches related to Coulomb branches in the dual theory can be realized in terms of non-toric and non-polynomial deformations. In particular the physical spectrum is not determined by the theoretical topological data of the Calabi-Yau manifold, but by the number of deformations of a specific toric realization of it.

\subsection{Toric geometry of the fibration}

As described in sect.3.3. we have to fiber the local geometry $\mathcal{W}_{2}$ over a $\mathbf{P}^{1}$. For the cases where $H \subset G_{0}$, with $G_{0}$ the heterotic gauge group, we can describe $\mathcal{W}_{2}$ as a local patch of the K3 $W_{2}$ given by a polyhedron $\Delta_{3}^{\star}$ as in Table 2. In this fibration, $\Delta_{3}^{\star}$ becomes the hypersurface $\mathcal{H}: \nu_{i, 1}^{\star}=0$ in a four-dimensional polyhedron $\Delta_{4}^{\star}$ corresponding to a Calabi-yau three-fold $W_{3}$. It remains to specify the vertices of $\Delta_{4}^{\star}$ which do not lie in $\mathcal{H}$.

There is no freedom in the choice of the base manifold; it is just a $\mathbf{P}^{1}$. In the toric polyhedron the vertices associated to the $\mathbf{P}^{1}$ are given by projecting along the fiber directions [27] which maps a vector $\nu_{i}^{\star}$ to its first entry. The vertices for the toric variety $\mathbf{P}^{1}$ are $\{(-1),(1)\}$. Thus we add vertices with first entry \pm 1 .

Consider now the base of the elliptic fibration $\pi_{F}: W_{3} \rightarrow B_{2}$. In the simplest case the base is a $\mathbf{P}^{1}$ bundle over a base $\mathbf{P}^{1}$ (with the base $\mathbf{P}^{1}$ being the base of the elliptic K3 $\left.W_{2}\right)$, that is a Hirzebruch surface $\mathbf{F}_{n}$. The vertices of $\mathbf{F}_{n}$ are $\nu_{i}^{\star} \in\{(-1,0),(0,-1),(0,1),(1, n)\}$, with the two relations between the $\nu_{i}^{\star}$ corresponding to the two classes of $\mathbf{P}^{1}$ 's. The vertices with $\nu_{i, 1}^{\star}=0$ are already contained in the hyperplane $\mathcal{H}$. We add therefore two vertices and obtain a polyhedron

$$
\Delta_{4}^{*}=\text { convex hull }\left\{\left(0, \nu_{i}^{\star \prime}\right),(1, n, 2,3),(-1,0,2,3)\right\}
$$

Furthermore, we can add two types of vertices corresponding to non-perturbative dynamics of the heterotic string: $a$ ) we can blow up the base $\mathbf{F}_{n}$ of the elliptic fibration $\pi_{F}$ where the new moduli associated to the blown up spheres correspond to non-perturbative tensor multiplets from five-branes in six dimensions [5], b) we can introduce singularities in the elliptic fibration 
located at points on the base $\mathbf{P}^{1}$. The new Kähler classes from reducible fibers correspond to non-perturbative gauge symmetries [5].

\subsection{The geometric moduli space}

Let us consider in more detail the precise meaning of the map $f: W_{3} \rightarrow Z_{2}$ which gives the heterotic manifold in terms of the polyhedron of the type IIA compactification. The moduli space of the type IIA compactification on $W_{3}$ has two sectors, the moduli space $\mathcal{M}_{H M}$ parametrized by the hypermultiplets and the vector multiplet moduli space $\mathcal{M}_{V M}$. These spaces are in general decoupled due to the constraints of $N=2$ supersymmetry up to subtleties explained e.g. in [28].

The hypermultiplets of type IIA on $W_{3}$ contain the string coupling, the complex structure of $W_{3}$ and the Ramond-Ramond (RR) fields. In the dual heterotic theory on $Z_{2} \times T^{2}$ the hypermultiplets contain the geometric moduli of $Z_{2}$ and the data of the bundle on $Z_{2}$. The complex structure of $W_{3}$ describes the geometry of $Z_{2}$ and part of the bundle data, namely the "spectral cover" $C$ of $V$ or its generalizations defined by $\mathcal{W}_{3}$ for $H \neq S U(n)$. The $\mathrm{RR}$ moduli determine a line bundle $\mathcal{L}$ on $C$ [8].

The 20 hypermultiplets that describe the geometry of K3 split into Kähler deformations and complex deformations in a given algebraic realization of $Z_{2}$. In particular, if $Z_{2}$ is elliptically fibered and has a global section, the Picard lattice $\operatorname{Pic}\left(Z_{2}\right)$ has at least rank two with a hyperbolic plane $U$ generated by the class of the section and the class of the elliptic fiber. We can therefore test only the part of the moduli space of K3 compactifications with rank $\operatorname{Pic}\left(Z_{2}\right) \geq 2$ using the type IIA/F-theory picture. Actually we can argue that it is sufficient to consider the case with rank $\operatorname{Pic}\left(Z_{2}\right)=2$. Namely, since Kähler and complex structure deformations of a singularity are equivalent for $\mathrm{K} 3$, we can always choose a complex deformation of a singularity to keep the rank of $P i c\left(Z_{2}\right)$ fixed ${ }^{10}$. Said differently, reaching a singularity in the complex structure of $Z_{2}$, there is in general no new branch in the moduli space corresponding to Kähler deformations of this singularity. However there can be new branches in four dimensions if the singularity is associated with non-perturbative gauge symmetries $\hat{G}$. In this case there is a new branch in the four dimensional compactification on $\mathrm{K} 3 \times T^{2}$ corresponding to non-vanishing $\hat{G}$ Wilson lines on $T^{2}$ emanating from the locus of singular K3.

\footnotetext{
${ }^{10}$ The same will of course not be true in the case of $n>2$, where Kähler and complex structure deformations are not equivalent.
} 
Keeping this in mind we can now make the meaning of the map $f$ from the type IIA to the heterotic polyhedron more precise. The correspondence of the geometric hypermultiplet moduli space, identifying the complex structure deformations of $W_{n+1}$ with the complex structure of $Z_{n}$ and the bundle data on it has been discussed in the previous sections. It remains to assign the vector moduli, corresponding to Kähler moduli of the toric manifold $W_{n+1}$, to heterotic deformations.

In general, the Kähler deformations of the toric variety $W_{n+1}$ defined by a complex polyhedron $\Delta_{W_{n+1}}^{*}$ provide always a complete resolution of the canonical singularities of birational equivalent models. In particular this implies also the complete resolution of singularities for any divisor in $W_{n+1}$. Let us assume that $\Delta_{Z_{n}}^{*}$ appears not only as a projection but is a hyperplane in $\Delta_{W_{n+1}}^{*}$, that is $Z_{n}$ is a divisor in $W_{n+1}$. The Kähler resolution of $W_{n+1}$ provides a Kähler resolution of the divisor $Z_{n}$. However, since the Kähler moduli of type IIA on $W_{3}$ correspond to vector multiplets whereas the Kähler deformations of the heterotic string on $Z_{2}$ are related to hypermultiplets, the Kähler blow up of the singularities in the divisor $Z_{2}$ of $W_{3}$ is not mapped to a Kähler blow up of $Z_{2}$. Rather it corresponds to the above mentioned four-dimensional Coulomb branch of non-perturbative gauge symmetries emanating from the singularity in $Z_{2}$. So although the Kähler resolution of $W_{3}$ provides a Kähler resolution of a singularity of $Z_{2}$, the correct heterotic picture is in terms of a Coulomb branch of a nonperturbative gauge symmetry compactified on the singular $\mathrm{K} 3 \times T^{2}$. Of course this interpretation is only possible because of the equivalence of the moduli space of Kähler deformations of singularities in the elliptic fibration of $\mathrm{K} 3$ manifolds with the moduli space of flat bundles on an elliptic curve! This is another, different consequence of the fact that we used elliptically fibered manifolds as the starting point for our geometric construction of flat bundles.

The fact that $\Delta_{Z_{n}}^{*}$ appears in general only as a projection rather than a hyperplane, fits nicely in the above picture. Since $Z_{2}$ does not represent a divisor in $W_{3}$, the complete Kähler resolution of $W_{3}$ does not necessarily provide a complete Kähler resolution of $Z_{2}$. This corresponds to the situation with generic bundle $V$, where the singularity in $Z_{2}$ does not lead to a nonperturbative gauge symmetry and there is no new branch in the moduli space. 


\subsection{Smooth bundles}

We consider now in some detail configurations which correspond to smooth heterotic bundles at a generic point in moduli space. To specify the theory we have to choose two bundles $V_{1}, V_{2}$ in the two $E_{8}$ factors and the integer $n$ which specifies the fibration of the K3 fiber $M_{2}$ over a further $\mathbf{P}^{1} \cdot n$ corresponds also to the way the total instanton number $k=24$ is divided between the two $E_{8}$ factors: $k_{1}=12+n, k_{2}=12-n$ [5] .

The geometry corresponding to the choice of $\left(V_{1}, V_{2}\right)$ and $n$ is as follows. The bundle $\left(V_{1}, V_{2}\right)$ determines the K3 fiber $M_{2}$. As before we take the structure group of $V_{2}$ to be trivial and concentrate on the first $E_{8}$ factor. For a structure group $H_{1}$ we take the corresponding $\mathrm{K} 3$ specified in Table 2. The instanton number $k_{1}$ is encoded in the fibration of $M_{2}\left(H_{1}\right)$ over the base $\mathbf{P}^{1}$ with coordinates $(s, t)$ and corresponds to the choice of $n$ for the Hirzebruch surface $\mathbf{F}_{n}$ [5]. We therefore consider the polyhedron $\Delta_{4}^{\star}$ of 5.1 with $n=k_{1}-12$.

$S U(N)$ bundles

From the polyhedron 5.1 we obtain a Calabi-Yau three-fold $W_{3}\left(A_{N}\right)$. The defining hypersurface is given by 3.1. Taking the local limit of $p_{\Delta_{4}^{*}}$ and making our special choice of local coordinates we obtain the local three-fold geometry $\mathcal{W}_{3}$ :

$$
\begin{aligned}
\left.p_{\Delta_{4}^{*}}\right|_{\text {local }} & =p_{0}+p_{+} \\
p_{0} & =y^{2}+x^{3}+\tilde{z}^{6} f_{12}+y \tilde{z}^{3} h_{6}+x \tilde{z}^{4} h_{8}+x^{2} \tilde{z}^{2} h_{4}+y x \tilde{z} s t \\
p_{+} & =v\left(\tilde{z}^{N} f_{k_{1}}+x \tilde{z}^{N-2} f_{k_{1}-4}+\ldots+\left\{\begin{array}{c}
x^{N / 2} f_{k_{1}-2 N} \\
y x^{\frac{N-3}{2}} f_{k_{1}-2 N}
\end{array}\right\}\right) .
\end{aligned}
$$

Here $f_{l}$ is a generic polynomial of homogeneous degree $l$ in the variables $(s, t)$ while $h_{l}$ is of the restricted form $h_{l}=s^{l}+\alpha_{l} t^{l}$. The interpretation of the three complex dimensional geometry $\mathcal{W}_{3}$ is very similar to the situation we encountered before: $v=0$ projects onto the K3 surface $Z_{2}: p_{0}=0$. This is the $\mathrm{K} 3$ surface (dual to the manifold) on which the heterotic string is compactified. Integrating out the linear variable $v$ we obtain a one-dimensional geometry, the intersection $p_{0}=0 \cap p_{+}=0$ which describes a curve $C$ in $Z_{2}$. $C$ is the spectral curve which determines the $S U(N)$ bundle on $Z_{2}$ as in $[8,10]$.

The number of parameters of $p_{+}$is $N k_{1}-N^{2}+2$. Discarding one parameter which can be absorbed in an overall rescaling this agrees with the dimension of the moduli space of $A_{N}$ bundles of instanton number $k$ on K3 
as determined by the index formula

$$
\operatorname{dim} \mathcal{M}(H)=c_{2}(H) k-\operatorname{dim}(H),
$$

which applies for simple $H$ and large enough $k$. Strictly speaking the formula (5.2) and similar formulae below for the other groups are valid for the values of $N$ which appear in Table 2 . However it is worth noting that these formulae are valid for any large $N$ as well. The only new aspect is that for large $N$ we have to consider non-compact Calabi-Yau geometries as in [11].

\section{$S O(2 N+1)$ bundles}

For the group $B_{N}$ (as well as for several other groups below) we encounter the situation that the fibered geometry $W_{3}(H)$ includes monomials with identical scaling properties in addition to those encountered for $W_{2}(H)$. In particular note that the polynomial $p_{+}^{1}$ has degree $N$ in the variables $(y, x, \tilde{z}) \sim\left(\lambda^{3} y, \lambda^{2} x, \lambda \tilde{z}\right)$ but does not contain all monomials of the appropriate weight. In the fibered geometry $W_{3}\left(B_{N}\right)$, the monomials in $W_{2}\left(B_{N}\right)$ are multiplied by general functions $f_{l}$ of the base variables $(s, t)$, while the extra monomials are multiplied by restricted functions $h_{l}=s^{l}+\alpha_{l} t^{l}$. The general expression can be written as

$$
\begin{aligned}
p_{+}^{1}= & \tilde{z}^{N} f_{k_{1}}+\tilde{z}^{N-3} y f_{k_{1}-6}+\epsilon y x^{\frac{N+1}{2}-2} f_{k_{1}-2 N}+(1-\epsilon) x^{\frac{N}{2}} f_{k_{1}-2 N}+ \\
& \tilde{z}^{N-2} x h_{k_{1}-4}+\tilde{z}^{N-4} x^{2} h_{k_{1}-8}+\tilde{z}^{N-5} x y h_{k_{1}-10}+\tilde{z}^{N-6} x^{3} h_{k_{1}-12} \\
& +\ldots+\epsilon \tilde{z} x^{\frac{N+1}{2}-1} h_{k_{1}-2 N+2}+(1-\epsilon) \tilde{z} y x^{\frac{N}{2}-2} h_{k_{1}-2 N+2} \\
p_{+}^{2}= & \tilde{z}^{2 N-6} f_{2 k_{1}-12}+\tilde{z}^{2 N-8} x f_{2 k_{1}-16}+\ldots+x^{N-3} f_{2 k_{1}-4 N}
\end{aligned}
$$

with $\epsilon=0$ (1) for $N$ even (odd). The number of parameters is $(2 N-1) k_{1}-$ $\left(2 N^{2}+N\right)+1$ as predicted by the index formula. The $S O(7)$ case is again obtained from $S O(9)$ by dropping the second term $x f_{2 k_{1}-16}$ in $p_{+}^{2}$.

\section{$S p(N)$ bundles}

The $S p(N)$ case can again be considered as a modding of the $S U(2 N)$ bundle by the operation $y \rightarrow-y$. This agrees with the result obtained from the geometric construction:

$$
p_{+}=v\left(\tilde{z}^{2 N} f_{k_{1}}+x \tilde{z}^{2 N-2} f_{k_{1}-4}+\ldots+x^{N} f_{k_{1}-4 N}\right) .
$$

The number of parameters is $(N+1) k_{1}-\left(2 N^{2}+N\right)+1$ as predicted by (5.3).

$S O(2 N)$ bundles

For $H=S O(2 N)$ we have again extra monomials because $p_{+}^{1}$ of $W_{2}\left(D_{N}\right)$ is 
not of the generic form. We find

$$
\begin{aligned}
p_{+}^{1}= & \tilde{z}^{N} f_{k_{1}}+\tilde{z}^{N-3} y f_{k_{1}-6}+\tilde{z}^{1-\epsilon} y x^{\frac{N+\epsilon}{2}-2} f_{k_{1}-2(N-1+\epsilon)} \\
& +\tilde{z}^{\epsilon} x^{\frac{N-\epsilon}{2}} f_{k_{1}-2(N-\epsilon)}+\tilde{z}^{N-2} x h_{k_{1}-4}+\tilde{z}^{N-4} x^{2} h_{k 1-8}+\tilde{z}^{N-5} x y h_{k_{1}-10} \\
& +\tilde{z}^{N-6} x^{3} h_{k_{1}-12}+\ldots+\tilde{z}^{3-\epsilon} y x^{\frac{N+\epsilon}{2}-3} h_{k_{1}-2(N+\epsilon-3)} \\
& +\tilde{z}^{2+\epsilon} x^{\frac{N-\epsilon}{2}-1} h_{k_{1}-2(N-2-\epsilon)}, \\
p_{+}^{2}= & \tilde{z}^{2 N-6} f_{2 k_{1}-12}+\tilde{z}^{2 N-8} x f_{2 k_{1}-16}+\ldots+\tilde{z}^{2} x^{N-4} f_{2 k_{1}-4(N-1)} .
\end{aligned}
$$

with $\epsilon=0$ (1) for $N$ even (odd). The number of parameters in $p_{+}^{1}$ and $p_{+}^{2}$ is $(2 N-2) k_{1}-\left(2 N^{2}-N\right)+1$ in agreement with the index formula (5.3).

$G_{2}$ bundles

For $G_{2}$ bundles we obtain a geometry $W_{3}\left(G_{2}\right)$ :

$$
\begin{aligned}
& p_{+}^{1}=\tilde{z}^{3} f_{k_{1}}+x \tilde{z} h_{k_{1}-4}+y f_{k_{1}-6}, \\
& p_{+}^{2}=f_{2 k_{1}-12} .
\end{aligned}
$$

The number of parameters is $4 k_{1}-14+1$ in agreement with (3.5).

$F_{4}$ bundles

For $F_{4}$ bundles, the complex geometry $W_{3}\left(F_{4}\right)$ takes the form

$$
\begin{aligned}
& p_{+}^{1}=\tilde{z}^{4} f_{k_{1}}+x \tilde{z}^{2} h_{k_{1}-4}+y \tilde{z} h_{k_{1}-6}+x^{2} f_{k_{1}-8}, \\
& p_{+}^{2}=\tilde{z}^{2} f_{2 k_{1}-12}+x f_{2 k_{1}-16} \\
& p_{+}^{3}=f_{3 k_{1}-24} .
\end{aligned}
$$

The number of parameters is $9 k_{1}-52+1$.

$E_{6}$ bundles

If we fiber the geometry $W_{2}\left(E_{6}\right)$ we obtain a three-dimensional manifold

$$
\begin{aligned}
& p_{+}^{1}=\tilde{z}^{5} f_{k_{1}}+\tilde{z}^{3} x h_{k_{1}-4}+\tilde{z}^{2} y h_{k_{1}-6}+\tilde{z} x^{2} f_{k_{1}-8}+y x f_{k_{1}-10}, \\
& p_{+}^{2}=\tilde{z}^{4} f_{2 k_{1}-12}+\tilde{z}^{2} x f_{2 k_{1}-16}+\tilde{z} y f_{2 k_{1}-18}, \\
& p_{+}^{3}=\tilde{z}^{3} f_{3 k_{1}-24} .
\end{aligned}
$$

The number of parameters is $12 k_{1}-78+1$, as expected.

$E_{7}$ bundles

For $H=E_{7}$, the local geometry $W_{3}\left(E_{7}\right)$ takes the form

$$
p_{+}^{1}=\tilde{z}^{5} f_{k_{1}}+\tilde{z}^{3} x h_{k_{1}-4}+\tilde{z}^{2} y h_{k_{1}-6}+\tilde{z} x^{2} h_{k_{1}-8}+y x f_{k_{1}-10},
$$




$$
\begin{aligned}
p_{+}^{2} & =\tilde{z}^{4} f_{2 k_{1}-12}+\tilde{z}^{2} x h_{2 k_{1}-16}+\tilde{z} y f_{2 k_{1}-18}+x^{2} f_{2 k_{1}-20} \\
p_{+}^{3} & =\tilde{z}^{3} f_{3 k_{1}-24}+\tilde{z} x f_{3 k_{1}-28} \\
p_{+}^{4} & =\tilde{z}^{2} f_{4 k_{1}-36}
\end{aligned}
$$

The number of parameters is $18 k_{1}-133+1$ and agrees with 5.3.

$E_{8}$ bundles

Finally we describe $E_{8}$ bundles over the $\mathrm{K} 3 Z_{2}: p_{0}=0$. The geometry $W_{3}\left(E_{8}\right)$ reads

$$
\begin{aligned}
& p_{+}^{1}=\tilde{z}^{5} f_{k_{1}}+\tilde{z}^{3} x h_{k_{1}-4}+\tilde{z}^{2} y h_{k_{1}-6}+\tilde{z} x^{2} h_{k_{1}-8}+y x h_{k_{1}-10} \\
& p_{+}^{2}=\tilde{z}^{4} f_{2 k_{1}-12}+\tilde{z}^{2} x h_{2 k_{1}-16}+\tilde{z} y h_{2 k_{1}-18}+x^{2} f_{2 k_{1}-20} \\
& p_{+}^{3}=\tilde{z}^{3} f_{3 k_{1}-24}+\tilde{z} x h_{3 k_{1}-28}+y f_{3 k_{1}-30} \\
& p_{+}^{4}=\tilde{z}^{2} f_{4 k_{1}-36}+x f_{4 k_{1}-40} \\
& p_{+}^{5}=\tilde{z} f_{5 k_{1}-48} \\
& p_{+}^{6}=f_{6 k_{1}-60}
\end{aligned}
$$

The number of parameters is $30 k_{1}-248+1$ as expected.

\subsection{Singular bundles and non-perturbatively enhanced gauge symmetries}

The heterotic vacua with smooth compactification manifold $Z_{H}$ and generic $H$ bundle described so far have unbroken gauge symmetries with group $G$, the commutant of $H$ in the perturbative original ten-dimensional gauge group $G_{0}$. Let us investigate the conditions under which the heterotic string acquires extra massless degrees of freedom of non-perturbative origin. Note that the local mirror geometry $\mathcal{W}_{3}$, which defines the map of moduli spaces, is part of an F-theory compactification on the small fiber limit of $W_{3}$. We can therefore use the F-theory knowledge of how to engineer nonperturbative gauge symmetries to obtain a blow-up of $W_{3}$ corresponding to a non-perturbative gauge symmetry $G_{n p}[5,19]$. This is done by wrapping a seven-brane on the fiber $\mathbf{P}^{1}$ of the base $B_{F}=\mathbf{F}_{n}$ of the elliptic fibration $\pi_{F}^{\prime}: W \rightarrow B_{F}$. The geometry $\mathcal{W}_{3}^{\prime}$ obtained from the blown up three-fold defines the heterotic data in the same way as for the case of smooth bundles and smooth $Z_{2}$. In the toric language this corresponds to adding a vertex of type $b$ ) in sect.5.1. Let us first establish the following general result:

$(+)$ Consider the $E_{8} \times E_{8}$ string compactified on an elliptically fibered $K 3$ with a singularity of type $\mathcal{G}$ at a point $s=0$ and a special gauge 
background $\hat{V}$. If the restriction $\hat{V}_{\mid E_{H}}$ to the fiber $E_{H}$ at $s=0$ is sufficiently trivial, the heterotic string acquires a non-perturbative gauge symmetry $G_{n p} \supset \mathcal{G}$.

Note that the above result is very similar to the case of the type IIA string on singularities. In this case we know that type IIA on singularities of 2-cycles acquires a non-perturbative gauge symmetry from D2-brane wrappings, under the condition that the background field $B$ vanishes [66] In the heterotic string, not surprisingly, the vanishing condition includes also the gauge fields. It would be interesting to have a geometric interpretation of this gauge symmetry enhancement as in the brane picture of the type IIA theory.

There are two comments in order. Firstly, since the geometric data of $W_{3}$ describe only a subset of the heterotic moduli corresponding to the spectral cover and its generalization for other gauge groups, but not the extra information of a line bundle $\mathcal{L}$ on it [8], the enhancement of gauge symmetry requires in addition appropriate values for these non-geometric moduli. Secondly, the non-perturbative gauge group is at least $\mathcal{G}$ for a gauge background of the restricted type described below and can be larger than $\mathcal{G}$, if additional restrictions on the behavior of $\hat{V}$ in a neighbourhood of the singularity are imposed. We will discuss such cases below.

The verification of the above claim is very simple using the fact that the bundle is defined on the Calabi-Yau $Z_{2}: p_{0}=0$. Addition of vertices of type $b$ ) amounts to a singularity $\mathcal{G}$ of the Calabi-Yau manifold $W_{3}$ over a point on the base $\mathbf{P}^{1}$. If $W_{3}$ is written in generalized Weierstrass form ${ }^{11}$

$$
p=y^{2}+x^{3}+y x \hat{z} a_{1}+x^{2} \hat{z}^{2} a_{2}+y \hat{z}^{3} a_{3}+x \hat{z}^{4} a_{4}+\hat{z}^{6} a_{6},
$$

the conditions for a singularity of type $\mathcal{G}$ have been analyzed using the Tate's formalism in [19]. The singularity at a point $s=0$ is determined by the powers of vanishing of the coefficients $a_{i} \sim s^{n_{i}}$ specified by a vector $\mathbf{n}=\left(n_{1}, n_{2}, n_{3}, n_{4}, n_{6}\right)$.

Since the polynomial $p_{0}$ consists of a subset of the polynomials in (5.12), the coefficients $a_{i, 0}$ of the generalized Weierstrass form of $Z_{2}$ fulfil the same singularity condition as $p$, so $Z_{2}$ has a $\mathcal{G}$ singularity. Moreover from the explicit form of the bundle moduli space associated to a structure group $H$ we see, that if the dependence of the $a_{i}$ is given by $\mathbf{n}$ then the leading behavior of the bundle is shown ${ }^{12}$ in Table 3 .

\footnotetext{
${ }^{11}$ Note that we use $(y, x, \hat{z})$ and $(y, x, \tilde{z})$ to denote the homogeneous coordinates of the elliptic fiber of the $n+1$-dimensional Calabi-Yau $W_{n+1}$ and the $n$ dimensional Calabi-Yau $Z_{n}$, respectively.

${ }^{12}$ We restrict to the subset of structure groups $H$, for which the toric resolution results
} 


\begin{tabular}{|c|cccccc|}
\hline$H$ & $v$ & $v^{2}$ & $v^{3}$ & $v^{4}$ & $v^{5}$ & $v^{6}$ \\
\hline$S U(2)$ & $s^{n_{4}} x$ & - & - & - & - & - \\
$S U(3)$ & $s^{n_{3}} y$ & - & - & - & - & - \\
$S U(4)$ & $s^{n_{2}} x^{2}$ & - & - & - & - & - \\
$S U(5)$ & $s^{n_{1}} y x$ & - & - & - & - & - \\
$S O(7)$ & $s^{n_{2}} x^{2}$ & $s^{n_{6}} \tilde{z}^{2}$ & - & - & - & - \\
$S O(9)$ & $s^{n_{2}} x^{2}$ & $s^{n_{4}} x$ & - & - & - & - \\
$S O(11)$ & $s^{n_{1}} x y$ & $s^{n_{2}} x^{2}$ & - & - & - & - \\
$S p(2)$ & $s^{n_{2}} x^{2}$ & - & - & - & - & - \\
$S O(8)$ & $s^{n_{2}} x^{2}$ & $s^{n_{4}} x$ & - & - & - & - \\
$S O(10)$ & $s^{n_{1}} y x$ & $s^{n_{4}} x$ & - & - & - & - \\
$G_{2}$ & $s^{n_{3}} y$ & $s^{n_{6}}$ & - & - & - & - \\
$F_{4}$ & $s^{n_{2}} x^{2}$ & $s^{n_{4}} x$ & $s^{n_{6}}$ & - & - & - \\
$E_{6}$ & $s^{n_{1}} y x$ & $s^{n_{3}} \tilde{z} y$ & $s^{n_{6}} \tilde{z}^{3}$ & - & - & - \\
$E_{7}$ & $s^{n_{1}} y x$ & $s^{n_{2}} x^{2}$ & $s^{n_{4}} x \tilde{z}$ & $s^{n_{6}} \tilde{z}^{2}$ & - & - \\
$E_{8}$ & $s^{n_{1}} y x$ & $s^{n_{2}} x^{2}$ & $s^{n_{3}} y^{3}$ & $s^{n_{4}} x$ & $s^{n_{6}} \tilde{z}$ & $s^{n_{6}}$ \\
\hline
\end{tabular}

Table 3:Behavior of the gauge background $\hat{V}$ near the $\mathrm{K} 3$ singularity.

For example consider the simplest case with a structure group $H_{1}=S U(2)$. The Calabi-Yau threefold $W_{3}\left(A_{1}\right)$ is defined by the polynomial

$$
\begin{aligned}
& v^{-1} \tilde{z}^{6} f_{24-k_{1}}+ \\
& v^{0}\left(y^{2}+x^{3}+y x \tilde{z} s t+x^{2} \tilde{z}^{2} h_{4}+y \tilde{z}^{3} h_{6}+x \tilde{z}^{4} h_{8}+\tilde{z}^{6} f_{12}\right)+ \\
& v\left(\tilde{z}^{6} f_{k_{1}}+x \tilde{z}^{4} f_{k_{1-4}}\right)
\end{aligned}
$$

where $h_{i}=s^{i}+t^{i}$ and $f_{i}$ is a generic degree $i$ polynomial as before. To obtain a non-perturbative gauge symmetry $\hat{G}=S U(2)$ we blow up the locus $y=x=s=0$ in $W_{3}$ :

$$
y=u y, \quad x=u x, \quad s=u s .
$$

This blow up is compatible with only a subset of the perturbations in 5.14 and we get a new manifold

$$
\begin{aligned}
& v^{-1} s^{2} \tilde{z}^{6} f_{22-k_{1}}+ \\
& v^{0}\left(y^{2}+x^{3} u+x^{2} \tilde{z}^{2} h_{4}+y \tilde{z}^{3} s h_{5}+x \tilde{z}^{4} s h_{7}+y x \tilde{z} u s t+\tilde{z}^{6} s^{2} f_{10}\right) \\
& v\left(\tilde{z}^{6} s^{2} f_{k_{1-2}}+x \tilde{z}^{4} s f_{k_{1-5}}\right)
\end{aligned}
$$

Here $h_{i}$ and $f_{i}$ are as before apart from the fact that $s$ is replaced by $s u$. Note that the heterotic manifold $Z_{2}: p_{0}=0$ has an $A_{1}$ singularity at $s=0$. The spectral cover has become

$$
p_{+}=s\left(s \tilde{z}^{2} f_{k_{1}-2}+x f_{k_{1}-5}\right)=0
$$

in a Weierstrass form as in (5.12). 
Let $S$ denote the class of the section of $Z_{2}$ and $F$ the class of the generic elliptic fiber. The intersections are $S^{2}=-2, F \cdot S=1, F^{2}=0$. The class of the spectral cover $\Sigma: p_{+}=0$ is then $2 S+k_{1} F$ which is generically a smooth connected curve. The bundle $\hat{V}$ described by 5.17 instead corresponds to a spectral cover with two components, $\Sigma=\Sigma_{1}+\Sigma_{2}$ with $\left[\Sigma_{1}\right]=F$ and $\left[\Sigma_{2}\right]=2 S+\left(k_{1}-1\right) F$.

A physical interesting case which features a supposedly self-dual heterotic string theory in six dimensions is the compactification of the heterotic $E_{8} \times E_{8}$ string with 12 instantons in each $E_{8}$ factor [29, 30]. It was argued in [30, 31] that unhiggsing of a perturbative $S U(N)$ is dual to a non-perturbative $S U(N)$ arising from $1+\frac{N}{2} S O(32)$ small instantons without vector structure at an $A_{1}$ singularity (using a duality between $S O(32)$ and $E_{8} \times E_{8}$ string on $\mathrm{K} 3)$. Here we see that the $E_{8} \times E_{8}$ picture of this gauge enhancement is in terms of a compactification on a $\mathrm{K} 3$ with $A_{N-1}$ singularity, rather than $A_{1}$, with a particular behavior of the vector bundle near the singularity specified in Table $3^{13}$.

\subsection{Near the tangent bundle}

The class with structure group $H_{1}=S U(2)$ described in eq.5.14 should contain also the standard embedding with the gauge background identical to the spin connection. Since the $S U(2)$ bundle is embedded in one $E_{8}$ factor we choose $k_{1}=24$. The spectral bundle reads then

$$
C: \tilde{z}^{2} f_{24}+x f_{20}=0
$$

with the 46-1 parameters corresponding to the well-known 45 deformations of the tangent bundle $T$ of K3. To get strictly the tangent bundle, first note that the restriction $V_{E_{H}}$ to the elliptic curve must be trivial due to the flatness of $T^{2}$. So we have to tune $f_{20}=0$. Moreover the only pathologies of $T_{\mid E_{H}}$ appear at the singular fibers of the elliptic fibration. These occur at the 24 zeros of the discriminant $\Delta_{24}=4 f_{8}^{3}+27 g_{12}^{2}$ of $p_{0}$, where $f_{8}$ and $g_{12}$ are related to the polynomials in 5.16 by a shift of variables that puts $p_{0}$ into Weierstrass form, $y^{2}+x^{3}+x \tilde{z}^{4} f_{8}+\tilde{z}^{6} g_{12}$. So we must choose $f_{24}=$ const. $\Delta_{24}$. From the point of $\mathrm{F}$-theory this geometry is identical to the one with 24 small instantons sitting at the singular fibers of the fibration. This agrees with the result in [26]. There the authors argue that the difference between the tangent bundle and the small instanton configuration is in the non-geometric moduli corresponding to RR fields in the type IIA theory.

\footnotetext{
${ }^{13}$ For a related phenomenon in compactifications on the tangent sheaf, see [26].
} 


\subsection{Non-perturbative equivalences}

We now will study some applications of the toric map $f: W_{3} \rightarrow Z_{2}$ to investigate a certain class of six-dimensional heterotic theories with large non-perturbative groups and interesting non-perturbative equivalences. To recap, we consider a type IIA/F-theory compactification on an elliptically Calabi-Yau three-fold $W_{3}$ which also has a K3 fibration whose fiber we denote by $W_{2}$. This is dual to the heterotic string compactified on $Z_{2}$. In the toric polyhedron $\left.\Delta_{W_{3}}^{\star} i\right)$ the fiber $W_{2}$ corresponds to a hyperplane $\mathcal{H}=\Delta_{W_{2}}^{\star}$ in $\left.\Delta_{W_{3}}^{\star}, i i\right)$ the heterotic K3 $Z_{2}$ appears as the projection $f: \Delta_{W_{3}}^{\star} \rightarrow \Delta_{Z_{2}}^{\star}$.

An interesting situation appears, if the projection $f$ results in a hyperplane $\mathcal{H}^{\prime}$, that is $W_{3}$ admits at the same time a $Z_{2}$ fibration. The two K3 fibrations imply that we have two different perturbatively defined heterotic theories in four dimensions, which are non-perturbatively equivalent ${ }^{14}$

Moreover, if the two K3 manifolds defined by the hyperplanes $\mathcal{H}$ and $\mathcal{H}^{\prime}$ share the same elliptic fiber, we can take the F-theory limit without interfering with the equivalence ${ }^{15}$. In this way we obtain two six-dimensional heterotic compactifications on $Z_{2}$ and $W_{2}$ which are non-perturbatively equivalent. These manifolds can be at rather different moduli, one being highly singular while the other being smooth, as we will see in the following example.

\section{4 small instantons on smooth $K 3$}

Let us start with the simplest case corresponding to a heterotic theory with 24 small $E_{8}$ instantons on a single point in a smooth $\mathrm{K} 3 Z_{2}$. The perturbative gauge group is $E_{8} \times E_{8}$. The K3 fiber of the three-fold $W_{3}$ is therefore the K3 $W_{2}$ with $E_{8} \times E_{8}$ singularity described by the polyhedron

$$
\Delta_{W_{2}^{E_{8} \times E_{8}}}^{*}=\text { convex hull }\left\{e_{2}, e_{3}, u_{4}, \tilde{u}_{4}\right\},
$$

where $W_{2}$ has an elliptic fibration with fiber $\Delta_{E_{1}}^{*}=$ convex hull $\left\{e_{2}, e_{3}, f_{1}\right\}$. Let $\nu^{*(\alpha)}$ denote an $n+1$ dimensional vertex obtained from an $n$ dimensional vertex $\nu^{\star}$ by adding a zero at the $\alpha$-th position. Since $W_{2}$ appears as a hyperplane $x_{1}=0$ in $\Delta_{W_{3}}^{*}$ in our conventions, we obtain $\Delta_{W_{2}^{E_{8} \times E_{8}}}^{*(1)}$ as the first piece of $\Delta_{W_{3}}^{*}$. We will refer to the K3 $W_{2}$ which is the fiber of the K3 fibration $W_{3} \rightarrow \mathbf{P}^{1}$ as the "fiber K3".

\footnotetext{
${ }^{14}$ The quite reverse situation is known to occur, in which two heterotic theories have the same perturbative spectrum while non-perturbatively they are different [32]

${ }^{15}$ To be precise, in order for the F-theory limit to work, we have to require that not only $\mathcal{H}^{\prime}$ but also the hyperplane $\mathcal{H}: \nu_{i, 1}^{\star}=0$ coincides with a projection (in the first coordinate).
} 
The smooth $\mathrm{K} 3$ of the heterotic string appears as the projection in the direction of the second coordinate. It is modeled by a polyhedron

$$
\Delta_{Z_{2}^{A_{0}}}^{*}=\text { convex hull }\left\{e_{2}, e_{3}, v_{0}, \tilde{v}_{0}\right\}
$$

We add therefore $\Delta_{Z_{2}}^{*(2)}$ to $\Delta_{W_{3}}^{*}$. We will refer to the "heterotic K3" $Z_{2}$, which is an elliptic fibration over the base $\mathbf{P}^{1}$ of the K3 fibration as the "base $\mathrm{K} 3$ ".

Finally we have to ensure convexity of $\Delta_{W_{3}}^{*}$. A discrete series of solutions corresponding to the situation where the instantons have been divided into two groups with $a+b$ and $24-a-b$ instantons is provided by

$$
\Delta_{W_{3}}^{*}=\text { convex hull }\left\{\Delta_{W_{2}^{E_{8} \times E_{8}}}^{*(1)} \cup \Delta_{Z_{2}^{A_{0}}}^{*(2)} \cup \tilde{\Delta}_{A_{0}^{(1)}}^{*} \cup \tilde{\Delta}_{A_{0}^{(2)}}^{*}\right\}
$$

with

$$
\begin{aligned}
& \tilde{\Delta}_{A_{1}^{(1)}}^{*}=\text { convex hull }\{(1, a, 2,3),(1,-b, 2,3)\}, \\
& \tilde{\Delta}_{A_{0}^{(2)}}^{*}=\text { convex hull }\{(-1,12-a, 2,3),(-1,-12+b, 2,3)\},
\end{aligned}
$$

with $0 \leq a, b \leq 12$. For simplicity we assume in the following that both groups contain a non-zero number of small instantons.

Note how simple the toric construction of the combined data for the bundle and the manifold is. It is also easy to show that the Calabi-Yau manifolds $W_{3}$ associated to $\Delta_{W_{3}}^{\star}$ give indeed the correct physics. Firstly, the hodge numbers are $h^{1,1}=43(0), h^{1,2}=43(22)$, where the number in parentheses denotes the number $\delta h^{1,1}\left(\delta h^{1,2}\right)$ of so-called non-toric (nonpolynomial) deformations, which are not available in the toric model. The $n_{T}+n_{V}=h^{1,1}-2$ vector and tensor multiplets are associated to the 16 vector multiplets of $E_{8} \times E_{8}, 24$ tensor multiplets from the 24 small $E_{8}$ instantons and the heterotic coupling ( 2 Kähler classes corresponding to the volume of the elliptic fiber and the volume of the base do not contribute to the vector and tensor multiplets [5]). The $n_{H}=h^{2,1}+1$ hypermultiplets arise from the 20 moduli from K3 and 2 moduli for the two positions of the two groups of fivebranes. The 22 missing complex structure moduli correspond naturally to the fact that we have fixed 22 of 24 positions of the small instantons in the $\mathrm{K} 3 Z_{2}$.

There is a second elliptic fibration of the K3 fiber $W_{2}$ due to the hyperplane $\Delta_{E_{2}}^{*}=$ convex hull $\left\{e_{3}, w_{3}, \tilde{w}_{3}\right\}$ corresponding to the gauge group $S O(32)$ [33] Instead of $n_{T}=24$ extra tensors we have in this case an $S p(a+$ 
b) $\times S p(24-a-b)$ gauge group from two groups of coincident $S O(32)$ five branes [34] with matter in the $(\underline{2 k}, \underline{32}) \oplus(\underline{1}, \underline{1}) \oplus\left(\underline{2 k^{2}-k-1}, \underline{1}\right)$ of each $S p(k) \times S O(32)$ factor.

The non-perturbative gauge groups are determined by the intersections of the holomorphic two-cycles in $W_{3}{ }^{16}$ For the elliptic and K3 fibered manifolds used in the present context, these intersections can be conveniently described by projections of the polyhedron $\Delta_{W_{3}}^{*}$ in the direction of the elliptic fiber [36] For $W_{3}$ we find in this way indeed a non-perturbative gauge group $\hat{G}=\emptyset$ for the $E_{1}$ fibration and $S p(a+b) \times S p(24-a-b)$ for the $E_{2}$ fibration. Note that after having chosen the perturbative gauge group corresponding to $W_{2}$ and the heterotic compactification $Z_{2}$, the non-perturbative dynamics is completely determined by convexity of the polyhedron $\Delta_{W_{3}}^{*}$, with a discrete set of solutions corresponding to various branches in the moduli space.

These are the first two interpretations of F-theory compactification on $W_{3}$. Note that we have to shrink different elliptic fibers $E_{1}$ and $E_{2}$ in the F-theory limit, so these theories are disconnected in the small fiber limit in six dimensions and become equivalent only in five dimensions by T-duality [37].

\subsection{A non-perturbatively equivalent heterotic theory}

More interestingly, since the base $\mathrm{K} 3 \Delta_{Z_{2}^{A_{0}}}^{*}$, corresponding to the heterotic compactification manifold, does not only appear as a projection but as a hyperplane, there is a second $\mathrm{K} 3$ fibration with fiber $Z_{2}$ which is itself elliptically fibered with the same elliptic fiber $E_{1}$ as the K3 fiber of the original K3 fibration. Therefore we obtain a theory in six dimensions which is nonperturbatively equivalent to the heterotic string with 24 small instantons on on smooth $\mathrm{K} 3$.

We interpret now the smooth K3 described by ${\Delta_{Z_{2}}^{A_{0}}}$ as the fiber K3. Due to the absence of a singularity, the perturbative gauge group must be trivial and therefore the bundle $V_{0}$ has structure group $E_{8} \times E_{8}$ on the generic elliptic fiber. On the other hand, in the new K3 fibration, $W_{2}^{E_{8} \times E_{8}}$ has become the base K3. The heterotic compactification manifold has therefore an $E_{8} \times E_{8}$ singularity. The perturbative $E_{8} \times E_{8}$ gauge symmetry of the compactification with small instantons is produced in the dual theory purely by non-perturbative effects related to the singularities of the manifold and the bundle. For $a=b=12$ we have therefore the following duality:

\footnotetext{
${ }^{16}$ This is explained in detail in $[11,35]$
} 
(†) The $E_{8} \times E_{8}$ heterotic string compactified on a smooth K3 with two groups of 12 small instantons is non-perturbatively equivalent to compactifying on a K3 $p_{0}=0$ with $E_{8} \times E_{8}$ singularity with gauge bundle $V_{0}$. Here

$$
p_{0}=y^{2}+x^{3}+y x \tilde{z} s t+\tilde{z}^{6}\left(s^{7} t^{5}+s^{6} t^{6}+s^{5} t^{7}\right) .
$$

The $E_{8} \times E_{8}$ bundle $V_{0}$ is specified by a geometry $\mathcal{W}$ as in eq. 5.11 of the special form

$$
p_{+}=\tilde{v}\left(z^{15}+y x\right)+\tilde{v}^{2}\left(z^{14}+x^{2}\right)+\tilde{v}^{3}\left(z^{13}+y\right)+\tilde{v}^{4}\left(z^{\prime 2}+x\right)+\tilde{v}^{5} z^{\prime}+\tilde{v}^{6} .
$$

with $\tilde{v}=v s t, z^{\prime}=\tilde{z} s t$ and a similar polynomial for $p_{-}$for the other $E_{8}$ factor.

\section{Small instantons on singular K3 manifolds}

The above situation can be very easily generalized to singular K3 manifolds. Let us consider the case where we still start with only small instantons, now on a singular $\mathrm{K} 3$ surface. This case has been analyzed from various points of view in $[38,24]$. What is new is that with our understanding of bundles and manifolds we will sometimes find non-perturbatively equivalent theories involving a specific gauge background with non-trivial structure group on a different $\mathrm{K} 3$.

The construction of the appropriate three-folds $W_{3}$ is by now standard using our kit of $\mathrm{K} 3$ polyhedra: to obtain a compactification on a singular $\mathrm{K} 3$ we choose simply the polyhedron of the base K3 to describe a K3 manifold with a given singularity $G^{\prime}$. Requiring convexity of the polyhedron $\Delta_{W_{3}}^{*}$ starting from $\Delta_{Z_{2}^{G^{\prime}}}^{*(2)} \cup \Delta_{W_{2}^{E_{8} \times E_{8}}}^{*(1)}$ we obtain a set of discrete solutions corresponding to a choice of positions for the small instantons. It is impressive to observe that in fact all gauge groups derived in $[38,24]$ arise in the toric construction as a very simple consequence of the convexity of $\Delta_{W_{3}}^{*}$ !

We will discuss only one further example, the prototype case with an $A_{1}$ singularity in $Z_{2}$. This will turn out to be interesting also from its surprising relation to the compactification on the vector bundle near the tangent bundle. For $\Delta_{Z_{2}}^{*}$ we take the K3 with $A_{1}$ singularity

$$
\Delta_{Z_{2}^{A_{1}}}^{*}=\text { convex hull }\left\{e_{2}, e_{3}, v_{0}, v_{1}, \tilde{v}_{0}\right\}
$$

The solutions to the convexity with the 24 small instantons collected in two groups located at the singular point of $Z_{2}$ and a smooth point, respectively, are given by

$$
\Delta_{W_{3}}^{*}=\text { convex hull }\left\{\Delta_{W_{2}^{E_{8} \times E_{8}}}^{*} \cup \Delta_{Z_{2}^{A_{1}}}^{*} \cup \tilde{\Delta}_{A_{1}}^{*} \cup \tilde{\Delta}_{A_{0}}^{*}\right\},
$$


where the polyhedra $\tilde{\Delta}_{A_{1}}^{*}$ and $\tilde{\Delta}_{A_{0}}^{*}$ encode the information about the small instantons at the singular and smooth point of $\mathrm{K} 3$, respectively:

$\tilde{\Delta}_{A_{1}}^{*}=$ convex hull $\{(1, a, 2,3),(1, a-2,1,2),(1,-b, 2,3),(1,-b+2,1,2)\}$,

$\tilde{\Delta}_{A_{0}}^{*}=$ convex hull $\{(-1,12-a, 2,3),(-1,-12+b, 2,3)\}$,

with $2 \leq a, b \leq 12$. This configuration corresponds to a collection of $A=a+b$ instantons on the $A_{1}$ singularity and $B=24-A$ instantons on a smooth point in $\mathrm{K} 3$.

Determining the gauge symmetry from projecting along the fibers as in [36] we find the gauge groups

$$
\begin{aligned}
& E_{8} \times E_{8} \times S U(2)_{n p}^{A-3}, n_{T}^{\prime}=24, \\
& S O(32) \times S p(A)_{n p} \times S p(A-4)_{n p} \times S p(B)_{n p}, n_{T}^{\prime}=1,
\end{aligned}
$$

for the elliptic fibers $E_{1}$ and $E_{2}$, respectively. Moreover $n_{T}^{\prime}$ denotes the number of tensor multiplets in addition to the generic one. This is in agreement with the results of [38] and [24]. For the local geometry we have a chain of three ALE spaces fibered over three $\mathbf{P}^{1}$ 's with intersections between the neighbors. From the intersections we obtain matter as in [39] in addition to the gauge fields.

The heterotic manifold and the bundle $V$ on it can be obtained in the now familiar way from (3.1) and (2.4). For the manifold we obtain

$$
p_{0}=y^{2}+x^{3}+x^{2} z^{2} h_{4}+y z^{3} s h_{5}+x z^{4} s h_{7}+z^{6} s^{2} f_{10}+y x z\left(s t+t^{2}\right),
$$

which has an $A_{1}$ singularity at $s=0$. For the bundle we obtain

$$
p_{+}=v s^{A}, \quad p_{-}=v^{-1} t^{B} .
$$

Let us mention two cases which are physically interesting. The first case is where all instantons are on the $A_{1}$ singularity, $A=24$. We will argue in the next section that this theory is equivalent to a compactification on the deformation of the tangent bundle of a smooth $\mathrm{K} 3$ after compactification on a $T^{3}$. There is no non-perturbative dual in six dimensions since the projection in the $\nu_{i, 1}^{*}$ direction does not yield a K3 polyhedron.

As a second case consider $A=12$. This time there is a non-perturbative dual and it is in terms of an $E_{8} \times E_{7}$ bundle on a K3 with $E_{8} \times E_{8}$ singularity with a similar structure as in eq. 5.23. Quite generally it follows from the construction that the new duality implies:

(††) The heterotic string compactified on a $K 3$ with $G^{\prime}$ singularity and with a certain gauge background $V_{0}$ with structure group $H$ is nonperturbatively equivalent to the heterotic string compactified on a K3 
with $G$ singularity and with a specific gauge background $\tilde{V}_{0}$ with structure group $H^{\prime}$.

Here $H\left(H^{\prime}\right)$ is the commutant of $G\left(G^{\prime}\right)$ in $E_{8} \times E_{8}$. Technically, this duality exists if the F-theory manifold is described by a polyhedron with two K3 hyperplanes that coincide at the same time with projections and intersect in a plane that corresponds to an elliptic curve. The bundles $V_{0}$ and $\tilde{V}_{0}$ are determined by local mirror symmetry, as was done in the two examples above.

\subsection{F-theory on mirror manifolds}

It is instructive to look also at the heterotic theory corresponding to type IIA/F-theory compactification on the mirror manifold $M_{3}$ of $W_{3}$. In particular, upon further compactification on a circle to three dimensions, the two type IIA theories on $M_{3} \times S^{1}$ and $W_{3} \times \hat{S}^{1}$ should describe the same physics in virtue of mirror symmetry of Calabi-Yau three-fold and T-duality on the circle ${ }^{17}[40]$. Assuming that $M_{3}$ is also elliptically fibered, we have a pair of six-dimensional theories from F-theory compactification on $M_{3}$ and $W_{3}$. We do not expect these two theories to be dual in any sense in six dimensions. However, these theories are equivalent after compactification on a three torus to three dimensions. Using the toric map $f: W_{3} \rightarrow Z_{2}$ and some basic properties of mirror symmetry of $\mathrm{K} 3$ we can easily derive the six-dimensional theories which become equivalent after further compactification. Recall the claim $(*)$ concerning mirror symmetry of elliptically fibered K3 manifolds in sect.3.2. We can combine this result with the previous construction of Calabi-Yau three-folds to obtain a map between six-dimensional F-theories. We use $X_{2}^{H}, Z_{2}^{\hat{H}}$ to denote the two K3 manifolds used as the K3 fiber and the model for heterotic $\mathrm{K} 3$, respectively. Here $H$ and $\hat{H}$ denote the singularity type in the elliptic fibration. Consider the family of Calabi-Yau manifolds $X_{3}\left(X_{2}^{H}, Z_{2}^{\hat{H}}\right)$ which is constructed from choosing a convex closure of the vertices

$$
\Delta_{X_{2}^{H}}^{*(1)} \cup \Delta_{Z_{2}^{H}}^{*(2)} \subset \Delta_{4}^{\star}
$$

as before. This manifold describes a heterotic theory on $\mathrm{K} 3 Z_{2}$ with $\hat{H}$ singularity with perturbative gauge group $H$. Depending on which vertices are added to those in 7.4 to render $\Delta_{4}^{\star}$ convex, there can be also a non-perturbatively dual theory with the roles of $H$ and $\hat{H}$ exchanged. From the above we have that the mirror manifold of $X_{3}\left(X_{2}^{H}, Z_{2}^{\hat{H}}\right)$ is of type

\footnotetext{
${ }^{17}$ Here $\hat{S}^{1}$ denotes the T-dual of $S^{1}$.
} 


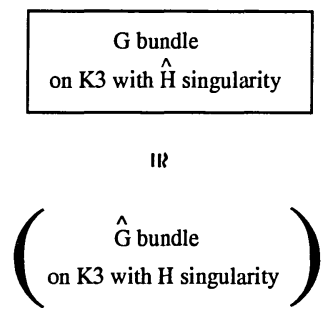

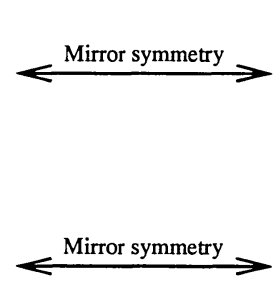

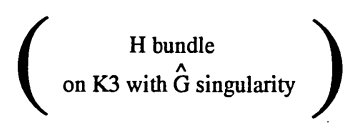

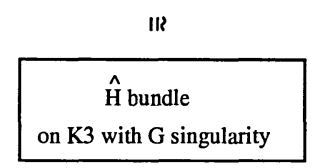

Figure 2:

$\tilde{X}_{3}\left(X_{2}^{G}, Z_{2}^{\hat{G}}\right)$. Thus mirror symmetry relates the heterotic theories in figure 2 .

Note that the formal mirror of the theory with $G$ bundle on K3 with $\hat{H}$ singularity is the one with an $H$ bundle on K3 with $\hat{G}$ singularity singularity (the two theories in the upper half of the diagram above). However if we do not insist on the special case of double K3 fibrations, the situation is different. The K3 fiber $X_{2}$ appears as a hyperplane in the three-fold polyhedron, while the heterotic $\mathrm{K} 3 Z_{2}$ as a projection. Moreover existence of a hyperplane (projection) in $\Delta^{*}$ corresponds to a projection (hyperplane) in $\Delta$. In particular this means that the $\mathrm{K} 3$ fiber and heterotic manifold are exchanged after the mirror transformation. The only generic K3 fibration in the mirror $\tilde{X}_{3}$ is the one with a fiber that is the mirror of the base $\mathrm{K} 3$ of $X_{3}$. The two relevant theories are denoted by a box in the above diagram.

As an example consider the case of a smooth $G$ bundle on a smooth K3, that is $\hat{H}=\emptyset$. The compactification on the mirror is a heterotic theory with 24 small $E_{8}$ instantons on a $G$ singularity ${ }^{18}$.

As a second example consider the compactification with 24 small instantons on the $A_{1}$ singularity described in the previous section. Using mirror symmetry we can show that the compactification on the mirror manifold describes an $S U(2)$ bundle with instanton number 24 on a smooth K3. In fact from (3.1), the defining equations for the heterotic compactification is $p=p_{0}+p_{+}+v^{-1}$, with

$$
\begin{aligned}
p_{0} & =y^{2}+x^{3}+\tilde{z}^{6} f_{12}+y \tilde{z}^{3} h_{6}+x \tilde{z}^{4} h_{8}+x^{2} \tilde{z}^{2} h_{4}+y x \tilde{z} s t, \\
p_{+} & =v\left(z^{2} f_{24}+x f_{20}\right),
\end{aligned}
$$

in agreement with eq. 5.2. Note that from 5.27 we have $h^{1,1}\left(W_{3}\right)=$ $h^{1,2}\left(M_{3}\right)=64$ from the $16+21$ vector multiplets, 24 tensors and three

\footnotetext{
${ }^{18} \mathrm{~A}$ relation between the instanton configurations of the heterotic string theories dual to F-theory compactifications on a Calabi-Yau 3-fold and its mirror has been conjectured in [41] based on a comparison of topological data.
} 
classes for the elliptic fiber and the two complex-dimensional base. In the mirror these deformations correspond to $65=h^{1,2}+1$ hypermultiplet moduli, splitting in the 20 geometric hypermultiplets from K3 and $45=2 \cdot 24-3$ moduli for the $S U(2)$ bundle. Similarly we have $h^{1,2}\left(W_{3}\right)=h^{1,1}\left(M_{3}\right)=18$, with the 19 hypermultiplets of $W_{3}$ corresponding to the K3 moduli minus one for the $A_{1}$ singularity. In the mirror, $h^{1,1}=18$ corresponds to the 15 vectors from $E_{8} \times E_{7}$ and the generic three classes.

For the case $A=12$, we find that the compactification on the mirror corresponds to a generic $k=12 S U(2)$ bundle on a smooth K3 plus 12 small instantons:

$$
p_{+}=v\left(z^{2} f_{12}+x f_{8}\right), \quad p_{-}=v^{-1} f_{12} .
$$

Note that the instanton number $k$ is determined by the number of small instantons on the $A_{1}$ singularity. However, there is a subtlety in the toric realization which follows from a simple counting of vector and hypermultiplets. For the theory on $W_{3}$ we get $52=3+16+9+24$ vector multiplets and $(20-1)+1$ hypermultiplets for a configuration with the 12 instantons on the same smooth point. This corresponds to a toric manifold $W_{3}$ with hodge numbers $h^{1,1}=52(0), h^{1,2}=30(11)$. The mirror with a $k=12$ $S U(2)$ bundle for a configuration with the 12 instantons on a single smooth point should have $n_{V}+n_{T}=30=3+15+12, n_{H}=42=20+21+1$ corresponding to $h^{1,1}=30(0), h^{1,2}=52(11)$. On the contrary the mirror of $W_{3}$ has $h^{1,1}=30(11), h^{1,2}=52(0)$, that is a deficit of 11 vector/tensor multiplets. This is related to the fact that the three-dimensional mirror symmetry, which relates the theories after compactification on $T^{3}$, exchanges Higgs and Coulomb branches. We now turn to a more detailed analysis of the relation between a particular physical model and the toric representation.

\subsection{Higgs branches in toric geometry and self-dual string dy- namics}

With the above understanding, we can refine the map between six-dimensional theories and determine the non-trivial mapping between various Higgs and Coulomb branches. Interestingly we will find that the hodge numbers of the Calabi-Yau manifold do not in general give the physical spectrum. We will still find complete agreement between toric manifolds and physics using a subtle realization of Higgs branches in toric geometry in terms of non-toric and non-polynomial deformations $\delta h^{1,1}$ and $\delta h^{1,2}$, respectively. Recall that these terms denote the number of Kähler and complex structure deformations, which are moduli of the general Calabi-Yau geometry $X$, but frozen in a given toric realization. We find that the deformations of the toric repre- 
sentation, $h_{e f f}^{i, 1}=h^{i, 1}-\delta h^{i, 1}$ agree precisely with the physical spectrum. In this sense the toric representation has more physical meaning than the theoretical geometric data. This is very similar to the F-theory duals of the CHL string [42] and confirms the picture that the choice of toric representation with non-toric and non-polynomial deformations has an important physical interpretation rather than being a technical subtlety [43]

Let us return to the example of $A$ small instantons on the $A_{1}$ singularity. In general, from 5.27 , we would expect

$$
h^{1,1}=40+A, \quad h^{1,2}=18+1,
$$

where the +1 denotes the extra position for the instantons on the smooth point if $A \neq 24$, which we assume for the following. Rather than this simple result, the polyhedra in eq. 5.26 correspond to Calabi-Yau manifolds $W_{3}$ with hodge numbers $h^{1,1}=40+A, h^{1,2}=18+B$. To be consistent with 5.33 there must be $\delta h^{1,2}=B-1$ non-polynomial deformations of $W_{3}$. The actual story is much richer: there is a chain of Calabi-Yau manifolds with this Hodge number but different numbers of $\delta h^{1,1}, \delta h^{1,2}$ and gauge symmetries shown in Tables 4,5 .

Let us finally comment on the other theories with a different number of effective moduli. The polyhedra for the theories in Table 4 are obtained by successively dropping the edge vertex of the line $\mu_{i}, i=1 \ldots B+1$ associated to the $B$ small instantons on a single smooth point. In the first step we loose one vertex from that line and catch a new point $p=(0,-1,0,0)$ in the K3 polyhedron. In the $E_{8} \times E_{8}$ language we have lost a tensor multiplet. The new point $p$ can not correspond to a tensor multiplet from a small instanton since the associated divisor does not give a location on the base.

\begin{tabular}{|l|cl|cc|}
\hline \multicolumn{1}{|c|}{$G=G_{n p} \times S O_{32}, n_{T}^{\prime}=1$} & $n_{T}^{\prime}$ & \multicolumn{1}{c|}{$G=G_{n p} \times E_{8} \times E_{8}$} & $h_{\text {eff }}^{1,1}$ & $h_{\text {eff }}^{1,2}$ \\
\hline$S p_{A} \times S p_{A-4} \times S p_{B}$ & 24 & $\left(S U_{2}\right)^{A-3}$ & $40+A$ & 19 \\
$\tilde{S U}_{2}^{(1)} \times S p_{A} \times S p_{A-4} \times S p_{B-1}$ & 23 & $\tilde{S U}_{2}^{(1)} \times\left(S U_{2}\right)^{A-3}$ & $40+A$ & 20 \\
$\tilde{S U}_{2}^{(2)} \times S p_{A} \times S p_{A-4} \times S p_{B-2}$ & 22 & $\tilde{S U}_{2}^{(2)} \times\left(S U_{2}\right)^{A-3}$ & $39+A$ & 21 \\
$\vdots$ & $\vdots$ & $\vdots$ & $\vdots$ & $\vdots$ \\
$\tilde{S U}_{2}^{(B-1)} \times S p_{A} \times S p_{A-4} \times S p_{1}$ & $A+1$ & $\tilde{S U}_{2}^{(B-1)} \times\left(S U_{2}\right)^{A-3}$ & $18+2 A$ & $B+18$ \\
$\tilde{S U}_{2}^{(B)} \times S p_{A} \times S p_{A-4}$ & $A$ & $\tilde{S U}_{2}^{(B)} \times\left(S U_{2}\right)^{A-3}$ & $17+2 A$ & $B+18$ \\
\hline
\end{tabular}

Table. 4: Higgs branches emanating from type IIA compactification on $W_{3}$ with $A$ instantons on an $A_{1}$ singularity and $B=24-A$ small instantons on a smooth point. A superscript $S U_{2}^{(k)}$ denotes a level $k$ gauge group. 


\begin{tabular}{|c|c|c|c|}
\hline$G=G_{n p} \times S O_{28} \times S U_{2}, n_{T}^{\prime}=0$ & $n_{T}^{\prime} \quad G=G_{n p} \times E_{8} \times E_{7}$ & $h_{e f f}^{1,1}$ & $h_{e f f}^{1,2}$ \\
\hline$\tilde{S U}_{2}^{(B)}$ & $0 \quad \tilde{S U}_{2}^{(B)}$ & 19 & $40+A$ \\
\hline$S p_{1} \times \tilde{S U}_{2}^{(B-1)}$ & $\tilde{S U}_{2}^{(B-1)}$ & 20 & $40+A$ \\
\hline$S p_{2} \times \tilde{S U}_{2}^{(B-2)}$ & $2 \quad \tilde{S U}_{2}^{(B-2)}$ & 21 & $39+A$ \\
\hline$\vdots$ & $\vdots$ & $\vdots$ & $\vdots$ \\
\hline$S p_{B-1} \times \tilde{S U}_{2}^{(1)}$ & $B-1 \quad \tilde{S U}(1)$ & $B+18$ & $18+2 A$ \\
\hline$S p_{B}$ & $B$ & $B+18$ & $17+2 A$ \\
\hline
\end{tabular}

Table. 5: Higgs branches for the heterotic compactification on the mirror manifolds of those in Table 4.

Anomaly cancellation in six dimensions as well as the fact that the size of the Kähler class is blown down by the small fiber limit imply that the new branch exists in one lower dimension upon compactification on a circle. Blowing down the divisor corresponding to the point $p$ we get a gauge symmetry enhancement to a new $S U(2)$ factor that is denoted by a tilde in the Tables 4,5. In the following, continuing dropping vertices from the line $\mu_{i}$, at each step we loose one rank for the gauge group while the self-intersection of the sphere associated to $p$ jumps by -2 . We interpret this effect as a higher level rank gauge factor $\tilde{S U}(2)^{(k)}$.

In fact it is likely that these Higgs branches are associated to extra matter, charged under the circle $U(1)$, which arises in the $E_{8} \times E_{8}$ picture from self-dual string windings on $S^{1}$. In particular, as we will argue in a moment, the $E_{8}$ five-brane in six dimensions, when compactified on a torus, gives rise to matter in the fundamental representation of the $S U(2)$ enhanced gauge symmetry of the heterotic $E_{8}$ string at $T=U$. While the vector bosons in this case arise from fundamental string windings that become massless at $T=U$, we claim that the non-critical string compactified on the same torus gives rise to hypermultiplets, charged under the $S U(2)$, at the special radius. Whereas the massless vector states follow easily from the perturbative world-sheet formulation of the heterotic fundamental string, a similar phenomenon is somehow surprising for the non-critical string; in particular there is no known framework to describe the dynamics of the non-critical string and the generation of massless states at $T=U$.

To see that the five-brane wrapped on the torus produces a fundamental of the torus $S U(2)$, recall that geometrically, the five-brane is associated to a blow up of the base, which is a Hirzebruch surface $\mathbf{F}_{n} . \mathbf{F}_{n}$ is a $\mathbf{P}^{1}$ fibration over $\mathbf{P}^{1}$, which in the framework of geometric engineering of type IIA is well-known to give rise to a pure $S U(2)$ gauge theory [14]. To add matter in this theory, one blows up the $\mathbf{P}^{1}$ fibration at a point of the base [39]. 
But precisely the same is done in F-theory in six dimensions to get a tensor. Upon compactification on the torus, which unifies the two pictures, the size of the fiber $\mathbf{P}^{1}$ gets identified with the difference $T-U$ on the heterotic side [44] Thus the blow up corresponding to the $E_{8}$ fivebrane has become equivalent to the addition of a single doublet in the $S U(2)$ at $T=U$ of the heterotic string.

\section{$6 \quad N=1$ supersymmetric vacua in four dimensions}

We proceed now with the most interesting case of $N=1$ supersymmetric vacua in four dimensions. In sect.6.1 we give a systematic description of the bundle in terms of two line bundles $\mathcal{L}, \mathcal{N}$ on the base $B_{n-1}$. This determines essentially the $n+1$-fold geometries $\mathcal{W}_{n+1}$, whose precise form can be obtained from the local mirror limit. In sect.6.2 we explain the relation of these two bundles to similar bundles used in the construction of Friedman Morgan and Witten In sect.6.3 we give explicit discussion of the case of toric four-folds ${ }^{19}$ that are fibered over the toric bases $\mathbf{P}^{2}$ and $\mathbf{F}_{n}$, or blow ups thereof. In sect.6.4 we describe the geometry for compactifying on (deformations of) the tangent bundle for any dimension $n$. In sect. 6.5 we describe how to construct bundles on singular Calabi-Yau three-folds and describe new non-perturbative dualities that are similar to the ones discussed in six dimensions.

\subsection{Description of the Bundle}

Our construction of the local geometry $\mathcal{W}$ does not depend on the dimension of the base $B_{n-1}$. As before we get the equation for the mirror geometry from 3.1 and taking the local mirror limit 2.4, or directly from 3.2. Below we describe the outcome in a more economic way in terms of the eightdimensional geometries of sect.4. This general discussion will cover much of the information of the detailed result.

The two-fold geometries of sect.4 are defined by the vanishing of the polynomial

$$
p_{\mathcal{W}}=p_{0}+p_{+}=0 .
$$

The polynomial $p$ depends on four coordinates $(y, x, \tilde{z}, v)$. In the higherdimensional fibration, the coefficients of the polynomials in $p$ will become functions of the variables of the base $B_{n-1}$. More precisely, $(y, x, \tilde{z}, v)$ become

\footnotetext{
${ }^{19}$ Toric constructions of Calabi-Yau 4-folds have been discussed in $[45,46,47,48,49]$.
} 
sections of line bundles on $B_{m-1}$. These line bundles correspond to the scaling relations $l^{(r)}$ of the polyhedron $\Delta^{\star}$, as discussed in sect.3.1. If we know these scalings, we can determine the generic dependence of the function $f^{c, d}$ multiplying a term $y^{a} x^{b} \tilde{z}^{c} v^{d}$ of the eight-dimensional local geometry.

Naively we have to determine four different line bundles for the four variables $(y, x, \tilde{z}, v)$. However we have one scaling relation 4.2 amongst them. Let us call the line bundle associated to it $\mathcal{O}$. Moreover we have assumed that the elliptic fibration has a section which gives a second constraint. In total we have therefore to specify $2=4-2$ line bundles on $B_{n-1}$ to upgrade the two-dimensional local geometry $W_{2}$ to a $n+1$-dimensional local geometry $\mathcal{W}_{n+1}$.

Let us denote these two bundles by $\mathcal{M}$ and $\mathcal{L}$. By definition, $y$ is a section of $\mathcal{O}^{3}, y \in \Gamma\left(\mathcal{O}^{3}\right)$. Since we have the terms $y^{2}$ and $x^{3}$ appearing in $p$, we must have $y \in \Gamma\left(\mathcal{A}^{3} \mathcal{O}^{3}\right), x \in \Gamma\left(\mathcal{A}^{2} \mathcal{O}^{2}\right)$ and $p \in \Gamma\left(\mathcal{A}^{6} \mathcal{O}^{6}\right)$, for a line bundle $\mathcal{A}$. From the fact that

$$
p_{0}=y^{2}+x^{3}+x \tilde{z}^{4} f+\tilde{z}^{6} g+\ldots
$$

describes a Calabi-Yau manifold $Z_{n}$, with $f, g$ polynomials in the base variables, we need to have $\mathcal{A}=\mathcal{M L}$, with $\tilde{z} \in \Gamma(\mathcal{M O})$ and $\mathcal{L}$, the anticanonical bundle of $B_{2}$. Moreover from $p_{+} \in \Gamma\left(\mathcal{M}^{6} \mathcal{L}^{6} \mathcal{O}^{6}\right)$ we have $v \in$ $\Gamma\left(\mathcal{M}^{5-N} \mathcal{O}^{6-N}\right)$, with $N$ being the highest $z$ power in $p_{+}^{1}$. This fixes almost completely the four-dimensional local geometry $\mathcal{W}_{n+1}$ in terms of $\mathcal{W}_{2}$ :

$$
\begin{aligned}
\mathcal{W}_{2} & \rightarrow \mathcal{W}_{n+1} \\
y^{a} x^{b} \tilde{z}^{c} v^{d} a_{c, d} & \rightarrow y^{a} x^{b} \tilde{z}^{c} v^{d} f^{c, d} \\
f^{c, d} & \in \Gamma\left(\mathcal{M}^{d} \mathcal{L}^{c}\right) .
\end{aligned}
$$

Note that the fact that $a=0,1$ and $3 a+2 b+c=6$ implies that the coefficient function $f_{c, d}$ has only two indices, which is equivalent to our previous statement that the definition involves only two independent line bundles. Eq. 6.3 gives a general definition of the line bundle $\mathcal{M}$ for any fibration, also for non-toric bases. We will give explicit expressions for toric bases in sect.6.3.

\subsection{More on the line bundle $\mathcal{M}$ and stability of $V$}

Let us compare the above result from mirror symmetry with the general structure of the moduli space of holomorphic stable vector bundles found in [8]. Friedman, Morgan and Witten described holomorphic stable bundles on elliptically fibered Calabi-Yau manifold $Z$ by fibering the data $\left(E, W^{r}\right)$ 
holomorphically over a complex base $B$. Here $W^{r}$ is a short-hand notation for the weighted projective space $\mathbf{W} \mathbb{P}_{s_{0}, \ldots, s_{r}}^{r}$ predicted by Loojienga. The projective spaces $W^{r}$ fit into a holomorphic bundle $\underline{W}^{r}$ over $B$. If $s: B \rightarrow$ $\underline{W}^{r}$ is a section, the homogeneous coordinates $\tilde{a}_{i}$ of $\underline{W}^{r}$ pull back to sections

$$
\tilde{a}_{i} \in H^{0}\left(B, \mathcal{N}^{s_{i}} \otimes \mathcal{L}^{-d_{j}}\right),
$$

where $\mathcal{L}$ is the anti-canonical bundle of $B, \mathcal{N}$ a line bundle on $B$, the $d_{j}$ are the degrees of the independent Casimir operators of the group $H$ and $s_{i}$ the Dynkin indices as above. Part of the data of $V$ are defined by choosing a section of $W^{r}$. The line bundle $\mathcal{N}$ is an important characteristic of $V$ and its first Chern class $\eta=c_{1}(\mathcal{N})$ is closely related to the higher Chern classes of $V[8,56]$

Our local mirror construction has given a similar answer for the structure of the bundle: the relation between the geometry and the associated topological data is essentially determined by the two line bundles $\mathcal{L}$ and $\mathcal{M}$. Of course the two descriptions should agree and in fact it is easy to see that eqs. 6.3 and 6.4 imply

$$
\mathcal{N}=\mathcal{M L}^{6} .
$$

Eq. 6.3 gives a general definition of the line bundle $\mathcal{N}$ in terms of the toric construction for all structure groups $H$ as well as singular configurations such as sheafs and geometric singularities. In particular we see that $\mathcal{N}$ is described in toric terms as a simple linear relation $l^{(\mathcal{N})}$ between the vertices of the toric polyhedron $\Delta^{\star}$. As we will see in a moment, convexity of a polyhedron $\Delta^{\star}$ that describes a fixed structure group imposes a constraint on the possible values of $\eta=c_{1}(\mathcal{N})$.

\subsection{Toric Bases}

To get a four-dimensional theory by considering the F-theory compactification associated to the local mirror limit of the type IIA geometry we fiber the local geometries $\mathcal{W}_{2}$ over a two complex dimensional base $B_{2} . B_{2}$ will also be the base of the elliptic fibration $\pi_{H}: Z_{3} \rightarrow B_{2}$ of the Calabi-Yau three-fold $Z_{3}$ on which the bundle $V$ is defined. A toric representation can be given for the cases $B_{2}=\mathbf{P}^{2}$ or $\mathbf{F}_{n}$, or a series of blow ups thereof. With these two choices for $B_{2}$ the toric polyhedra $\Delta_{B_{2}}^{\star}$ are the convex hull of the vertices

\begin{tabular}{c|c|c}
$\mathbf{P}^{2}$ & $\mu_{i}^{\star} \in \Delta_{B_{2}}^{\star}$ & $K$ \\
\hline$s$ & $(1,0)$ & 1 \\
$t$ & $(0,1)$ & 1 \\
$u$ & $(-1,-1)$ & 1
\end{tabular}




\begin{tabular}{c|c|cc}
$\mathbf{F}_{n}$ & $\mu_{i}^{\star} \in \Delta_{B_{2}}^{\star}$ & $K_{1}$ & $K_{2}$ \\
\hline$t$ & $(0,1)$ & 1 & $-n$ \\
$s$ & $(0,-1)$ & 1 & \\
$t^{\prime}$ & $(1,0)$ & & 1 \\
$s^{\prime}$ & $(-1, n)$ & & 1
\end{tabular}

Here we have also indicated the scaling relations between the vertices and associated coordinates on them. These coordinates transform as sections of the line bundles defined by the scaling relations ${ }^{20}$, e.g. $t^{\prime} \in \Gamma\left(\mathcal{K}_{1}^{0} \otimes \mathcal{K}_{2}^{1}\right)$.

As before, the three-fold base $B_{3}$ of the elliptic fibration $\pi_{F}: W_{4} \rightarrow$ $B_{3}$ will determine the topological properties of the gauge bundle $V$ on the Calabi-Yau $Z_{3}$. The base $B_{3}$ is a $\mathbf{P}^{1}$ bundle over $B_{2}$ with the following toric data. From $B_{2}=\mathbf{P}^{2}$ we get a $\mathbf{P}^{1}$ bundle that we call $\tilde{\mathbf{F}}_{k}$ :

\begin{tabular}{c|c|cc}
$\tilde{\mathbf{F}}_{k}$ & $\mu_{i}^{\star} \in \Delta_{B_{3}}^{\star}$ & $K_{0}$ & $K$ \\
\hline$z$ & $(0,0,1)$ & 1 & $-k$ \\
$w$ & $(0,0,-1)$ & 1 & \\
$s$ & $(0,1,0)$ & & 1 \\
$t$ & $(1,0,0)$ & & 1 \\
$u$ & $(-1,-1, k)$ & & 1
\end{tabular}

The integer $k$ specifies the $\mathbf{P}^{1}$ bundle over $\mathbf{P}^{2}$. Similarly we get from $B_{2}=\mathbf{F}_{n}$ a $\mathbf{P}^{1}$ bundle $\tilde{\mathbf{F}}_{k, m, n}$ :

\begin{tabular}{c|c|ccc}
$\tilde{\mathbf{F}}_{k, m, n}$ & $\mu_{i}^{\star} \in \Delta_{B_{3}}^{\star}$ & $K_{0}$ & $K_{1}$ & $K_{2}$ \\
\hline$z$ & $(0,0,1)$ & 1 & $-k$ & $-m$ \\
$w$ & $(0,0,-1)$ & 1 & & \\
$t$ & $(0,1,0)$ & & 1 & $-n$ \\
$s$ & $(0,-1, k)$ & & 1 & \\
$t^{\prime}$ & $(1,0,0)$ & & & 1 \\
$s^{\prime}$ & $(-1, n, m)$ & & & 1
\end{tabular}

with the two integers $k, m$ specifying the $\mathbf{P}^{1}$ fibration

The four-fold $W_{4}$ will be defined by a polyhedron $\Delta_{5}^{\star}$ which is obtained by joining the vertices of the base $B_{2}$ and the vertices of the K3 polyhedron $\Delta_{H}^{\star} 3.6$ which describes the $H$ bundle:

$$
\Delta_{5}^{\star}=\text { convex hull }\left\{\Delta_{H}^{*(1,2)} \cup \tilde{\Delta}_{B_{3}}^{\star}\right\} .
$$

Here the superscript $(1,2)$ denotes adding zeros at the first and second position of the K3 vertices in 3.6 and $\tilde{\Delta}_{B_{3}}^{\star}=\left\{\nu^{\star}: \nu^{\star}=\left(\mu^{\star}, 2,3\right), \mu^{\star} \in \Delta_{B_{3}}^{\star}\right\}$.

\footnotetext{
${ }^{20} \mathrm{~A}$ letter $K$ denotes the class of the line bundle $\mathcal{K}$.
} 
We can add three kinds of vertices to the polyhedron (some of which may be enforced by the convexity condition); $a^{\prime}$ ) blow ups of the $\mathbf{P}^{1} \rightarrow B_{2}$ fibration. These are similar to case $a$ ) in six dimensions and correspond to non-perturbative tensor multiplets from five-branes in the heterotic dual; $b^{\prime}$ ) extra singularities of the elliptic fibration located on the base $B_{2}$ related to non-perturbative gauge dynamics of the heterotic dual; $c^{\prime}$ ) blow ups of the base $B_{2}$. Note that due to the eight-dimensional equivalence the base $B_{2}$ is visible to both the heterotic string and the F-theory. Thus a modification of $B_{2}$ is common to both theories. As for the two line bundles appearing in 6.3 , we have

$$
\begin{aligned}
& \mathcal{L}\left(\mathbf{P}^{2}\right)=3 K=3 D_{s}, \\
& \mathcal{L}\left(\mathbf{F}_{n}\right)=2 K_{1}+(2-n) K_{2}=2 D_{s}+(2-n) D_{s^{\prime}},
\end{aligned}
$$

with $D_{x}: \quad x=0$. The bundle $\mathcal{M}$ is determined by the transformation properties of, say, $y$ under the rescalings of $\Delta^{\star}$. Consider first $\tilde{\mathbf{F}}_{k}$. Including homogeneous coordinates $(y, x, \hat{z})$ for the elliptic fiber, the polyhedron $\Delta_{5}^{\star}$ has scaling relations

\begin{tabular}{l|cccccccc} 
& $y$ & $x$ & $\hat{z}$ & $z$ & $w$ & $s$ & $t$ & $u$ \\
\hline $\mathcal{O}$ & 3 & 2 & 1 & & & & & \\
$\mathcal{K}$ & $3 \lambda$ & $2 \lambda$ & & $-k$ & & 1 & 1 & 1
\end{tabular}

with $\lambda=3-k$. From the scaling properties of $y$ and $\mathcal{L}=\mathcal{K}^{3}$ it follows that $\mathcal{M}\left(\tilde{\mathbf{F}}_{k}\right)=-k \mathcal{K}$. The relation between the global toric coordinates in 6.12 and the coordinates $(y, x, z, v)$ of the local geometry in this case can be seen to be

$$
(y, x, \tilde{z}, v)_{l o c a l}=\left(y, x, \hat{z} z w, w z^{-1}(\hat{z} z w)^{6-N}\right)_{g l o b a l},
$$

with the result that $v \in \Gamma\left(\mathcal{M}^{5-N} \mathcal{O}^{6-N}\right)$ as promised. Similarly we find $\mathcal{M}\left(\tilde{\mathbf{F}}_{k, m, n}\right)=-k K_{1}-m K_{2}$. So for the bundle $\mathcal{N}$ in 6.5 we have

$$
\begin{aligned}
\tilde{\mathbf{F}}_{k}: & \mathcal{N}=(18-k) \mathcal{K}, \\
\tilde{\mathbf{F}}_{k, m, n}: & \mathcal{N}=(12-k) \mathcal{K}_{1}+(12-6 n-m) \mathcal{K}_{2} .
\end{aligned}
$$

\subsection{Heterotic $(2,2)$ compactification in $10-2 n$ dimensions}

Using the F-theory/heterotic map presented above it is straightforward to provide the F-theory manifold $X_{n+1}$ which corresponds to a heterotic compactification on the tangent bundle in $10-2 n$ dimensions. The heterotic gauge bundle takes values in the structure group $S U(n)$. A generic deformation of the tangent bundle is obtained by fibering the relevant $\mathrm{K} 3$ manifold in Table 2. The tangent bundle itself is a highly degenerate version of the 
general $S U(n)$ bundle for the same reasons as in sect.5.5: the tangent bundle on the generic elliptic curve is trivial, so the bundle (or rather sheaf) $V$ of the heterotic compactification must be trivial away from the discriminant locus of the fibration $\pi_{H}: Z_{n} \rightarrow B_{n-1}$. The only pathologies of $V$ appear at the discriminant locus of the fibration. In this way we get the following "theorem":

Let the heterotic string be compactified on the elliptically fibered CalabiYau $Z_{n}, \pi_{h}: Z_{n} \rightarrow B_{n-1}$ described in Weierstrass form by

$$
p_{H}=y^{2}+x^{3}+x z^{4} f+z^{6} g=0,
$$

where $f$ and $g$ are sections of $\mathcal{L}^{4}$ and $\mathcal{L}^{6}$, respectively. The spectral cover of the two components of the sheaf $V$ in the gauge group $E_{8} \times E_{8}$ is described by the equations

$$
p_{+}=z^{n} \Delta, \quad p_{-}=1,
$$

where $\Delta$ is the discriminant of the elliptic fibration $\pi_{H}, \Delta=4 f^{3}+$ $27 g^{2}$. This is dual to F-theory compactified on the Calabi-Yau $n+1$ fold $X_{n+1}$ given by

$$
p_{F}=y^{2}+x^{3}+x(\hat{z} z w)^{4} f+(\hat{z} z w)^{6} g+\hat{z}^{6} z^{5} w^{7} \Delta+\hat{z}^{6} z^{7} w^{5} .
$$

Here $(z, w)$ denote the variables parametrizing the base $\mathbf{P}^{1}$ of the elliptically fibered K3 fiber of the K3 fibration $\pi_{F}: X_{n+1} \rightarrow B_{n-1}$.Eqs. 6.16 and $6.17 \mathrm{fix}$ the K3 fibration structure of $X_{n+1}$ completely. In particular, from 6.16 and 6.3 we see that $f^{0,0,6,-1} \in \Gamma\left(\mathcal{M}^{-1} \mathcal{L}^{6}\right)$ must be a section of the trivial bundle so $\mathcal{M}=\mathcal{L}^{6}$. E.g., the standard embedding for the two base geometries $\mathbf{P}^{2}$ and $\mathbf{F}_{n}$ discussed in the previous section arises from F-theory on an elliptic fibration over $\tilde{\mathbf{F}}_{18}$ and $\tilde{\mathbf{F}}_{12,6(2-n), n}$, respectively.

\subsection{Bundles on singular manifolds and non-perturbative du- alities in four dimensions}

As in six dimensions, there are non-trivial non-perturbative dualities in four dimensions implied by the local mirror construction (and corresponding dualities will also exist in lower dimensions). Let $\Delta_{5}^{\star}$ describe the polyhedron associated to a Calabi-Yau manifold $W_{4}$. There is a codimension two hyperplane $\mathcal{H}=\Delta_{3}^{\star}$ associated to an elliptically fibered $\mathrm{K} 3, W_{2}$. The elliptic fiber is described by a hyperplane $\Delta_{2}^{\star}(E)$ in $\Delta_{3}^{\star}$. The $W_{2}$ fibration of $W_{4}$ describes $H$ bundles on a Calabi-Yau three-fold $Z_{3}$ given by a projection in the direction of the section of $W_{2}$. In our conventions the hyperplane is given by 
$x_{1}=x_{2}=0$ and the projection is in the third direction. A non-perturbative dual in four dimensions exists if $i$ ) there is a second codimension two hyperplane $\mathcal{H}^{\prime}$ that describes an elliptically fibered $\mathrm{K} 3$ manifold $W_{2}^{\prime}$ with a section and the same elliptic fiber $E$ as $W_{2}: \mathcal{H} \cap \mathcal{H}^{\prime}=\Delta_{2}^{\star}(E)$; ii) the projection in the direction of the section of $W_{2}^{\prime}$ results in a reflexive polyhedron for a Calabi-Yau three-fold $Z_{3}^{\prime}$.

We have:

$(\dagger \dagger \dagger)$ Let the heterotic string be compactified on a Calabi-Yau threefold with $G^{\prime}$ singularity and with a certain gauge background with structure group $H$ such that the toric data $\Delta_{5}^{\star}$ fulfil conditions $\left.i\right)$ and ii). Then there exists a non-perturbatively equivalent compactification on a Calabi-Yau manifold with $G$ singularity and with a specific gauge background with structure group $H^{\prime}$.

Again $H\left(H^{\prime}\right)$ is the commutant of $G\left(G^{\prime}\right)$ in $E_{8} \times E_{8}$.

Note that as in six dimensions it is very easy to describe $H$ bundles on Calabi-Yau manifolds with $G^{\prime}$ singularity from our construction. If $\Delta_{3}^{\star}$ is the polyhedron associated to $H$ bundles as in sect.3.2 and $\Delta_{4}^{\star}$ the polyhedron associated to the Calabi-Yau manifold $Z_{3}$ with a $G$ singularity, we consider polyhedra $\Delta_{5}^{\star}$ that provide a convex closure of the vertices

$$
\begin{aligned}
& \left(0,0, \mu_{i}\right), \mu_{i} \in \Delta_{3}^{\star}, \\
& \left(\rho_{i, 1}, \rho_{i, 2}, a_{i}, \rho_{i, 4}, \rho_{i, 5}\right), \rho_{i} \in \Delta_{4}^{\star}
\end{aligned}
$$

with $a_{i}$ integers that determine the topological data of the bundle. As a simple example consider a theory with a smooth $E_{8} \times E_{8}$ bundle on a CalabiYau manifold $\pi: Z_{3} \rightarrow \mathbf{F}_{0}$ with an $A_{1} \times A_{1}$ singularity in the elliptic fibration. The polyhedron is given by

$$
\Delta_{5}^{\star}=\Delta_{A_{0}}^{*(1,2)} \cup \Delta_{Z_{3}}^{*(3)}
$$

where we use the same notation as in sect.5. Explicitly, we have

$$
\begin{aligned}
\Delta_{A_{0}}^{*(1,2)} & =\text { convex hull }\{(0,0, \pm 1,2,3),(0,0,0,-1,0),(0,0,0,0,-1)\} \\
\Delta_{Z_{3}}^{*(3)} & =\left\{\begin{array}{c}
\text { convex hull }( \pm 1,0,0,2,3),(0, \pm 1,0,2,3),(0, \pm 1,0,1,2) \\
(0,0,0,-1,0),(0,0,0,0,-1)
\end{array}\right\}
\end{aligned}
$$

These data correspond to a toric Calabi-Yau manifold with $h^{1,1}\left(\delta h^{1,1}\right)=$ $6(0), h^{1,2}=0, h^{1,3}\left(\delta h^{1,3}\right)=2130(0)$ and $\chi=12864=0 \bmod 24$. Note that we have three non-perturbatively equivalent theories corresponding to a $\mathrm{K} 3$ 
fiber given by $x_{a}=x_{b}=0$ and a projection to a Calabi-Yau manifold $Z_{3}$ in the $c$ direction:

- $(a, b, c)=(1,2,3):$ An $H=E_{8} \times E_{8}$ bundle on an elliptically fibered Calabi-Yau $\pi: Z_{3} \rightarrow \mathbf{F}_{0}$ with $A_{1} \times A_{1}$ singularity;

- $(a, b, c)=(1,3,2):$ An $H=E_{7} \times E_{7}$ bundle on an elliptically fibered Calabi-Yau $\pi: Z_{3} \rightarrow \mathbf{F}_{0}$ without singularity;

- $(a, b, c)=(2,3,1):$ An $H=E_{8} \times E_{8}$ bundle on an elliptically fibered Calabi-Yau $\pi: Z_{3} \rightarrow \mathbf{F}_{0}$ with $A_{1} \times A_{1}$ singularity.

One also easily can construct dualities where all three theories are on different Calabi-Yau manifolds, and moreover such that the latter are fibered over bases $\mathbf{F}_{n}$ with different $n$.

\section{Phenomenological $N=1 d=4$ F-theory/heterotic Vacua}

Let us finally apply our framework to construct dual pairs of F-theory/heterotic vacua with phenomenologically interesting gauge bundles. In particular, we will consider $S U(N)$ bundles in one $E_{8}$ factor for $1 \leq N \leq 6$ on elliptically fibered Calabi-Yau three-folds with bases $B_{2}=\mathbf{P}^{2}$ or $\mathbf{F}_{n}$ corresponding to unbroken gauge groups $E_{8}, E_{7}, E_{6}, S O(10), S U(5)$ and $S U(3) \times S U(2)^{21}$.

\subsection{Moduli and Spectra of the Four-dimensional Theories}

The moduli of F-theory on Calabi-Yau 4-folds and the heterotic string dual on Calabi-Yau 3-fold appear in several ways in the different formulations according to whether they arise geometrically or non-geometrically, perturbatively or non-perturbatively.

The perturbative moduli of the heterotic string are

i) the complex structure of $Z_{3}$,

ii) the Kähler structure of $Z_{3}$,

iii) the moduli of the "spectral cover" $C$,

\footnotetext{
${ }^{21}$ Note that these gauge groups can be further broken by the non-geometric moduli.
} 
$i v$ ) the moduli of a line bundle $\mathcal{L}$ on the resolved fiber product $C \times_{B_{2}} Z_{3}$.

Clearly, $i$ ) and $i i i)$ correspond to the complex structure of $W_{4}$. There is one further complex structure modulus of $W_{4}$ that we lost in the local mirror limit. It is related to the Kähler class of the heterotic elliptic fiber.

The moduli $i v$ ) should be identified with instanton bundles on coinciding seven branes [10]. Note that an instanton inside a seven brane corresponds to an Euclidean three brane wrapped on the interior part of the seven brane [50].The specification of $\mathcal{L}$ includes the behavior on the exceptional divisors of the resolution of the fiber product $C \times_{B_{2}} Z_{3}$. Moreover, continuous moduli arise from non-trivial elements in $H^{1}(C)$. They correspond to elements in $H^{1,2}\left(W_{4}\right)$ [8]. In addition one can twist the Jacobian $H^{1,2}\left(W_{4}, \mathbf{R}\right) / H^{1,2}\left(W_{4}, \mathbf{Z}\right)$ by elements in $H^{2,2}\left(X_{4}, \mathbf{Z}\right)[8,64]$. This twisting contributes to the gravitational anomaly [51], as has been discovered in the M-theory context in terms of four-form flux [52].The equivalent contribution in F-theory arises from the instanton bundle in the seven brane due to the world-sheet couplings[53].

\section{Non-perturbative gauge symmetries}

As for the remaining Kähler moduli of $Z_{3}$, the $h^{1,1}\left(B_{2}\right)$ moduli in the base maps to the same moduli in F-theory, since the base is common to both theories in the compactification from eight dimensions. We are left with the Kähler moduli of $Z_{3}$ that arise neither from the base $B_{2}$ nor from the ellipitc fiber, that is reducible fibers from singularities of the elliptic fibration. We will now argue that they are equivalent to deformations of non-perturbative gauge dynamics of the heterotic string.

Let us first consider a simple case, where we have a non-perturbative gauge symmetry $G$ in the F-theory manifold $W_{4}$ from a $G$ singularity in the elliptic fibration over a divisor $D$ with codimension one in the base $B_{2}$. The singularity can be described in the Weierstrass form 5.12 in terms of the vanishing of the coefficients $a_{i}$. As argued in sect.5.4, if $D$ is toric, then the vanishing conditions on the $a_{i}$ are shared by the polynomial $p_{0}$ specifying the heterotic manifold; that is, $Z_{3}$ has (at least) a $G$ singularity. The Kähler blow up of this singularity in $Z_{3}$ can not correspond to a similar blow up in $W_{4}$, since in the F-theory limit these moduli are frozen to zero. However, there are other natural moduli associated to the $G$ singularity in $W_{4}$, namely the moduli of a non-trivial instanton bundle on the coinciding seven branes associated to the singularity over $D$. They are the only possible candidates for the dual of the heterotic moduli. Note that since the heterotic nonperturbative gauge symmetry $G$ is associated to the singular geometry, we 
indeed expect that the blow up moduli are charged with respect to $G$ and can be used to break the gauge symmetry.

In the reverse direction we can argue as follows. Assume we have a smooth $E_{8} \times E_{8}$ bundle on an elliptic fibration $Z_{3}$ with a singularity $G$ over a curve $C$ in $B_{2}$. From the above identifications there are no perturbative moduli available in the F-theory which could correspond to the Kähler blow up of $G$ in $Z_{3}$. The only moduli that are not ruled out by the perturbative argument are moduli from instantons on coinciding seven branes on a "nonperturbative" divisor $D$.

There is another possible scenario starting from the singular heterotic manifold in a case where there are no moduli from instantons on coinciding seven branes associated to non-perturbative gauge symmetries: there is an obstruction to blow up in the heterotic theory. This is reminiscent of a similar situation in the formulation of heterotic compactifications in terms of linear sigma models, where it can happen that one cannot extend a gauge background defined on the singular manifold to another one on the resolved manifold within this framework [54].

\section{Non-perturbative five-branes}

To complete our dictionary, there are two further types of moduli of nonperturbative origin in the heterotic theory. Firstly, there are $h_{5 B}^{1,1}=h^{1,1}\left(B_{3}\right)-$ $h^{1,1}\left(B_{2}\right)$ moduli of the F-theory manifold that have no correspondence in the perturbative heterotic theory. From the analogy to five-branes in six dimensio ns they give rise to non-perturbative tensor multiplets in four dimensions associated to non-critical strings in the four-dimensional theory ${ }^{22}$. A toric vertex $\nu$ associated to $h_{5 B}^{1,1}$ is one of the type $a^{\prime}$ ) specified in sect.6.1 and the divisor (curve) in $B_{2}$ asspciated to $\nu$ is the supersymmetric 2-cycle on which the five brane is wrapped. This is clear in the case of $B_{2}=\mathbf{F}_{n}$ which can be considered as a compactification of a six-dimensional theory on the base $\mathbf{P}^{1}$ of $\mathbf{F}_{n}$. The six-dimensional tensor multiplet gives rise to an antisymmetric tensor $B_{\mu \nu}$ and a scalar $\phi$ in four dimensions which combine to an $N=1$ linear multiplet. Secondly, if the instanton bundle does not balance the gravitational anomaly associated to the curvature of $Z_{3}$, there are additional five branes wrapped on the elliptic fiber $E_{H}$ of $Z_{3}[8]$. There is a $U(1)$ field and three complex scalars associated to the five brane, which specify a position on $B_{2}$ and a Wilson line on $E_{H}$. They correspond to the positions of a D3 brane on $B_{3}$ in the F-theory context ${ }^{23}$.

\footnotetext{
${ }^{22}$ Such strings have been suggested as a new mechanism for low energy supersymmetry breaking in [46].

${ }^{23}$ In the M-theory picture, apart from the contribution from four-flux on $H^{2,2}\left(W_{4}\right)$ and membranes filling space-time, there appears to be a further contribution to the gravita-
} 
Non-perturbative gauge symmetries from non-toric bases Another non-perturbative effect arises from elements $h^{1,2}\left(B_{3}\right)$ which give rise to vector multiplets in four dimensions. This can be argued from considering a compactification on $S^{1}$ and comparison with M-theory, which leads to the following contribution of the hodge numbers of $W_{4}$ to the four-dimensional fields $[48]^{24}$

$$
\begin{aligned}
& n_{V}=\left(h^{1,1}\left(W_{4}\right)-h^{1,1}\left(B_{3}\right)-1\right)+h^{1,2}\left(B_{3}\right) \\
& n_{C}=h^{1,1}\left(B_{3}\right)+\left(h^{1,2}\left(W_{4}\right)-h^{1,2}\left(B_{3}\right)\right)+h^{1,3}\left(W_{4}\right) .
\end{aligned}
$$

In our case, since $B_{3}$ is toric, $h^{1,2}\left(B_{3}\right)=0$ and there are no contributions from $h^{1,2}\left(W_{4}\right)$ to vector multiplets. In the toric framework that we use these hodge numbers can be determined from the toric polyhedra $\Delta_{5}^{\star}$ and $\Delta_{5}$ using Batyrevs formula [57].

$$
\begin{aligned}
h^{1, i}= & \delta^{1, i}\left(l\left(\Delta^{\star}\right)-(n+2)-\sum_{\operatorname{codim} \theta^{\star}=1} l^{\prime}\left(\theta^{\star}\right)\right)+\sum_{\operatorname{codim} \theta^{\star}=i+1} l^{\prime}\left(\theta^{\star}\right) l^{\prime}(\theta) \\
& +\delta^{n-1, i}\left(l(\Delta)-(n+2)-\sum_{\operatorname{codim} \theta=1} l^{\prime}(\theta)\right) .
\end{aligned}
$$

Here $\theta$ denotes a face of $\Delta, \theta^{\star}$ the dual face and moreover $l$ and $l^{\prime}$ are the number of total and interior points of a face, respectively.

\subsection{Fibrations over $\mathrm{P}^{2}$}

Consider the case where the heterotic manifold is given by a fibration over $\mathbf{P}^{2}$. The compactification is specified by the choice of $N$ for the $S U(N)$ gauge bundle together with the integer $k$ that determines the base of the elliptic F-theory fibration $\tilde{\mathbf{F}}_{k}$. The requirement that the singularities in the ellipitic fibration are not worse than $E_{8}$ implies

$$
|k| \leq 18 \text {. }
$$

Note that this constraint gives a bound on the class $c_{1}(\mathcal{N})$ as can be seen from 6.14. We will discover a similar bound for non-trivial structure groups in a moment.

As in six dimensions, the number $k$ determines how the total Chern class $c_{2}(V)=c_{2}\left(V_{1}\right)+c_{2}\left(V_{2}\right)+[W]=c_{2}(T Z)$ of the gauge background is distributed among the $E_{8}$ factors. Here $[W]$ is the class of the fivebranes that

tional anomaly from five branes wrapped around 3-cycles in $W_{4}$ [46].

${ }^{24}$ See also ref.[55] 
are wrapped around the elliptic fiber [8] and are needed to ensure tadpole cancelations [9].

The starting point for type IIA manifolds $W_{4}$ is the polyhedron $\Delta_{5}^{\star}$ described in eq.6.10. In this convention, $k=18$ corresponds to a configuration near the standard embedding with the non-trivial gauge background embedded completely in one $E_{8}$ factor. Since the bundle in the second $E_{8}$ factor is trivial, we expect a gauge group $G_{N} \times E_{8}$ with $G_{N}$ the commutant of $S U(N)$ in $E_{8}$ and the extra $E_{8}$ factor arising from the second $E_{8}$. More generally, similar situations arise for large $k<18$ as a reflection of the fact that maximal breaking associated to the gauge background embedded in the second $E_{8}$ with fixed Chern classes terminates at a non-trivial extra gauge group $G^{(2)} \subset E_{8}$ (ingoring extra breaking due to non-geometric Higgs moduli). The gauge groups $G^{(2)}$ and the extra vertices $\mu_{i}$ which we add to $\Delta_{5}^{\star}$ to describe the toric resolution of the associated singularities are shown in Table 7.

Similarly if $k$ is small, the "instanton number" in the first $E_{8}$ bundle might not be sufficient to support the $S U(N)$ bundle for large $N$. This gives a restriction on possible combinations of $N$ and $k$. In Table B.1 In other words, there is a lower bound on $\eta=c_{1}(\mathcal{N})$ for a given structure group $S U(N)$.

In Table B.1 we collect the geometric data that enter the spectrum of the heterotic compactifications ${ }^{25}$. In particular we observe, that for a structure group $H=S U(N)$, we need $k \geq 3 N$ which means in view of 6.14 that

$$
\eta=c_{1}(\mathcal{N}) \geq N c_{1}(\mathcal{L}) \text {. }
$$

\begin{tabular}{|c|c|c|}
\hline$k$ & $G^{(2)}$ & $\mu_{i}$ \\
\hline 4 & $A_{1}$ & $(0,0,1,2,3),(0,0,1,1,2)$ \\
5,6 & $G_{2}$ & $(0,0,2,2,3)$ \\
$7,8,9$ & $F_{4}$ & $(0,0,3,2,3)$ \\
$10,11,12$ & $E_{7}$ & $(0,0,4,2,3),(0,0,3,1,2),(0,0,2,0,1),(0,0,1,0,0)$ \\
$13, \ldots, 18$ & $E_{8}$ & $(0,0,6,2,3)$ \\
\hline
\end{tabular}

Table 7: Singularities and toric resolution for $B^{(2)}=\mathbf{P}^{2}$.

The spectrum as determined by 7.1 is as follows: the vector multiplets follow from the gauge group $G_{N} \times G^{(2)}$. In many cases, for $N=1$ there is an

\footnotetext{
${ }^{25}$ Note that there is an irrelevant sign switch in our notation of $k$ as compared to sect. 6.3 .
} 
extra $\tilde{S U}(2)$ factor of the same origin as in the discussion of Higgs branches in six dimensions that explains the exceed of one in $h^{1,1}$. The hodge number $h^{1,2}$, which is expected to agree with the genus of the spectral cover, can be quite large for the manifolds under consideration.

\subsection{Fibrations over $F_{n}$ using Deformation of the Standard Embedding}

Let us now consider the case with $Z_{3}$ an elliptic fibration over $B_{2}=\mathbf{F}_{n}$. Requiring as before that the singularities of the ellipitc fibration are not worse than $E_{8}$ one obtains the restriction

$$
|k| \leq 12, \quad-(12+n(6+k)) \leq m \leq 12+n(6-k),
$$

which again defines a lower bound on $c_{1}(\mathcal{N})$ in 6.14 . We will consider only the case close to the standard embedding with the gauge background embedded in the $S U(N)$ bundle of a single $E_{8}$ factor, corresponding to $k=12$ and $m=12-6 n$.

The case $B_{2}=F_{n}$ is different from the $B_{2}=\mathbf{P}^{2}$ models discussed above in that for $|n| \geq 3$, the elliptic fibration over $B_{2}$ will have unavoidably singularities. Moreover the possible intersections of these singularities with those describing the perturbative gauge symmetries will lead to extra singularities above these intersections that need to be resolved in the Calabi-Yau four-fold $W_{4}$. In general this requires a case by case study of the actual singularities and their intersections. However we can give quite a canonical description for the following interesting combinations of $n$ and $N: i$ ) the trivial $S U(1)$ bundle for all $n ; i i) S U(N)$ bundles for $|n| \leq 6$.

Let us first consider the singularities associated to large $|n|$, in a heterotic compactification on the trivial $S U(1)$ bundle. The toric resolution of singularities in $W_{4}$ is described by adding the following vertices $\tilde{\nu}^{\star}$ to $\Delta_{5}^{\star}$ in 6.10: 


\begin{tabular}{|c|c|}
\hline$n$ & $\tilde{\nu}^{\star}$ \\
\hline$-12, \ldots,-9$ & $(0,-6,42,2,3)$ \\
$-8,-7$ & $(0,-4,30,2,3)$ \\
$-6,-5$ & $(0,-3,24,2,3)$ \\
-4 & $(0,-2,18,2,3)$ \\
-3 & $(0,-1,8,2,3)$ \\
$-2, \ldots, 2$ & - \\
3 & $(0,1,-4,2,3)$ \\
4 & $(0,2,-6,2,3)$ \\
5,6 & $(0,3,-12,2,3)$ \\
7,8 & $(0,4,-18,2,3)$ \\
$9, \ldots, 12$ & $(0,6,-30,2,3)$ \\
\hline
\end{tabular}

Table 8: Toric resolution for a trivial bundle on the fibration $\pi: Z_{3} \rightarrow \mathbf{F}_{n}$.

Taking the convex hull of these vertices leads to Calabi-Yau four-folds with the topological properties ${ }^{26}$ collected in Table B.2. For $k>2$ there are additional non-perturbative degrees of freedom that contribute to $h^{1,1}$ :

$$
\begin{aligned}
n=-3 \quad: \quad n_{T}^{\prime}=4, G_{n p}=S U(2), \\
n=-4 \quad: \quad n_{T}^{\prime}=6, G_{n p}=S U(2)^{2} \times G_{2}, \\
n=-5,-6 \quad: \quad n_{T}^{\prime}=10, G_{n p}=S U(2)^{2} \times G_{2}^{2} \times F_{4},
\end{aligned}
$$

Note that these spectra are precisely what we expect from $k=4,6,8$ small $E_{8}$ instantons on $E_{8}$ singularity, respectively.

For $N>1$ a toric resolution of the intersections of singularities can be described by further adding the vertices $\rho$ to $\Delta_{5}^{\star}$ :

\begin{tabular}{|c|c|}
\hline$n$ & $\rho$ \\
\hline$-6,-5$ & $(0,-1,4,2,3)$ \\
-4 & $(0,-1,6,2,3)$ \\
$-3, \ldots, 3$ & - \\
4 & $(0,1,-6,2,3)$ \\
5,6 & $(0,1,-8,2,3)$ \\
\hline
\end{tabular}

Table 9: Toric resolution for $S U(N)$ bundles on the fibration $\pi: Z_{3} \rightarrow \mathbf{F}_{n}$. The topological data for these cases are collected in Table B.3. ${ }^{27}$

\footnotetext{
${ }^{26}$ The Calabi-Yau manifolds associated to the polyhedra $\Delta_{5}^{\star}$ as defined above have identical hodge numbers for $n$ and $-n$ and we can therefore restrict to one sign for $n$.

${ }^{27}$ For $|n|=4$ and $N=2$ we have omitted the vertex $\rho$, since addition of the vertices $\tilde{\nu}^{\star}$ provides already a valid resolution.
} 
The non-perturbative degrees of freedom contributing to $h^{1,1}$ are

$$
\begin{array}{rll}
n=7,8 & : & n_{T}^{\prime}=14, G_{n p}=\tilde{S U}(2) \times S U(2)^{2} \times G_{2}^{3} \times F_{4}, \\
n=9, \ldots, 12 & : \quad & n_{T}^{\prime}=22, G_{n p}=\tilde{S U}(2) \times S U(2)^{4} \times G_{2}^{4} \times F_{4}^{2} \times E_{8}(7.7)
\end{array}
$$

These spectra are almost identical to what we expect from $k=9,10$ small $E_{8}$ instantons on an $E_{8}$ singularity, respectively. We interpret the modifications as deformations of these configurations.

\section{Conclusions}

We have seen how mirror symmetry can be used to define vector bundles on Calabi-Yau $n$-folds $Z_{n}$ and implies F-theory/heterotic duality at the classical level. In particular the construction allows for a very systematic identification of a dual pair realized in toric geometry, consisting of a Calabi-Yau $n+1$-fold $W_{n+1}$ for F-theory compactification and a Calabi-Yau $n$-fold $Z_{n}$ together with a family of vector bundles on it defining a heterotic theory. While the construction proves F-theory/heterotic duality at the classical level, we would really like to go on to a comparison of quantum corrected properties, both to verify duality at the quantum level and also to calculate quantities that are difficult to access in one of the two theories, assuming duality. There are two obvious candidates for quantum corrected quantities accessible by the geometric framework. Firstly, we can use mirror symmetry to compute correlation functions of two-dimensional topological sigma models[58] and compare to correlators in the space time theory. The general framework of mirror symmetry of Calabi-Yau $n$-folds has been developped in [59] and has been implemented into toric geometry in [46], with an identification of the relevant correlators in M-theory ${ }^{28}$.In general the correlators as computed by the topological sigma model are further modified by quantum corrections that do not arise from Euclidean fundamental string world-sheet wrappings. However, favorable situations in which these extra corrections are absent should exist and could be used for a comparison of heterotic and F-theory moduli spaces at the quantum level ${ }^{29}$.

A second important quantity of the four-dimensional $N=1$ theory is its superpotential for the moduli fields. In M-theory compactified on $W_{4}$ to

\footnotetext{
${ }^{28}$ For a relation to space time quantities in the two dimensional type IIA compactification, see[60].

${ }^{29} \mathrm{An}$ interesting comparison of quantum corrected correlators in eight dimensions appeared in [61].
} 
three dimensions, the superpotential arises from Euclidean five-brane wrappings on six-cycles $D$ with special topological properties [62], with an instanton action proportional to the volume $V(D)$ of $D$. For smooth $D$ the condition is that its arithmetic genus $\chi(D)$ is equal to one. This framework has been used in [63] to calculate the superpotential of $N=1$ field theories and their compactifications to three dimensions. In the toric Calabi-Yau manifolds used in the present paper, the divisors $D, \chi(D)$ and $V(D)$ can be systematically determined from intersection calculus [46]. It would be very interesting to apply and extend these toric methods in phenomenologically interesting $N=1 d=4$ theories constructed in this paper.

Acknowledgements: P.M. thanks S. Katz and C. Vafa for many valuable discussions related to [11]. The work of P.B. and P.M. was supported in part by the Natural Science Foundation under Grant No. PHY94-07194. P.B. would also like to acknowledge the Aspen Center for Physics and LBL, Berkeley for hospitality during the course of this work.

\section{Appendix A. The Local Mirror Limit}

To justify the limit 2.4 we have to show that it corresponds to the image in complex structure of the local limit in Kähler moduli space under the action of mirror symmetry. To decouple the Kähler deformations of the local resolution of an $H$ singularity in the elliptic fibration over a point $z=0$ on the base $\mathbf{P}^{1}$, we require first an infinite volume for the base and then concentrate on the flat local neighborhood of $z=0$.

The Kähler moduli $t_{r}, r=1, \ldots, h^{1,1}$ measure the volumes of holomorphic curves in $M_{n+1}$ with the volume form given by the Kähler form. In toric geometry, a non-trivial homology class corresponds to a linear relation $l^{(r)}$ between the vertices $\nu_{i}$ of the polyhedron $\Delta_{M_{n+1}}$ :

$$
\sum_{i} l_{i}^{(r)} \nu_{i}=0
$$

In the absence of non-toric deformations there are $h^{1,1}$ relations of this type. The specific choice of a basis of such linear relations which generates the Kähler cone is provided by the so-called Mori vectors.

Let us consider $n=1$, the case $n>1$ being completely analogous. For the K3 manifolds described in sect 3.2, the vertices $\nu_{i}$ can be divided into three groups $N_{\Sigma}, \Sigma \in\{0,+,-\}$ according to the sign of the first entry. The vertices with a zero entry form the polyhedron $\Delta_{E}$ of the elliptic fiber, while the vertices with positive (negative) first entry correspond to the resolution 
of a singularity $H_{1}\left(H_{2}\right)$ at $z=0(z=\infty)$, except for $\nu_{0}$ and $\tilde{\nu}_{0}$. The volumes of the generic elliptic fiber $E$ and the base $B$ correspond to the linear relations

$$
l^{(E)}:-6 e_{0}+2 e_{2}+3 e_{3}+f_{1}=0, \quad l^{(B)}: v_{0}+\tilde{v}_{0}-2 f_{1}=0,
$$

where $e_{0}=(0,0,0)$ is the single interior point of the polyhedron $\Delta_{M_{2}}$ and the coefficient of $e_{0}$ is determined by the fact that the vertices $\nu_{i}$ are in fact a short-hand notation for four-dimensional vertices in the hyperplane $x_{0}=1$ of a four-dimensional integral lattice $\Lambda_{4}[17]$.

The linear relation $l^{(B)}$ determines a holomorphic curve $C_{B}$ which is isomorphic to the base of the elliptic fibration of $M_{2}$. The local limit in Kähler moduli is thus given by $t_{B} \rightarrow i \infty$, where $t_{B}$ is the flat coordinate at large radius whose imaginary part measures the size of the base $B$.

In the K3 manifold $M_{2}$, a vertex $\nu_{i}$ of $\Delta_{M_{2}}$ corresponds to a divisor $D_{i}: x_{i}=0$ in $M_{2}$, where $x_{i}$ are the Batyrev-Cox variables used in the definition of the hyperplane as in 3.1. In particular, $C_{B} \subset M_{2}$ is given by $x_{f_{1}}=0$. There are further linear relations, or equivalently Kähler classes, $l^{(+, r)}$, which are associated to the resolution of the singularities $H_{1}$. They involve only vertices in $N_{0}$ and $N_{+}$. Similarly there is another set of linear relations $l^{(-, r)}$ involving vertices in $N_{0} \cup N_{-}$associated to the resolution of the $H_{-}$singularity. In terms of the mirror manifold $W_{2}$, the vertices $\nu_{i}$ correspond to deformations of the defining equation in 3.1. In particular a vertex $\nu_{i} \subset N_{\Sigma}$ corresponds to a monomial in $p_{\Sigma}$ in 2.3. To find the limit in complex structure corresponding to $t_{B} \rightarrow i \infty$ we need also the action of mirror symmetry on the moduli space, or said differently the map between Kähler moduli of $M_{2}$ and complex structure moduli of $W_{2}$. In the large radius/large complex structure limit it is given in terms of the so-called algebraic coordinates $z_{r}$ by

$$
t_{r}=\frac{1}{2 \pi i} \ln z_{r}, \quad z_{r}=\prod_{i} a_{i}^{l_{i}^{(r)}},
$$

where $a_{i}$ are the parameters for the complex structure related to the vertex $\nu_{i}$ as defined in 3.1 .

We are now ready to establish the validity of 2.4. We are searching for a limit $a_{i} \rightarrow \epsilon^{\lambda_{i}} a_{i}$ of the parameters $a_{i}$ in 3.1, defined by the exponents $\lambda_{i}$, which corresponds to $t_{B} \rightarrow i \infty$ for $\epsilon \rightarrow 0$, while keeping all the other Kähler moduli fixed. From A.3 it follows that $\lambda_{i}=\sum_{j} c^{j} \nu_{i, j}$ leaves all $z_{r}$ invariant, with $\nu_{i, j}$ denoting the $j$-th entry of the vertex $\nu_{i}$ and $c^{j}$ constants. However there is another solution for the $\lambda_{i}$ that leaves invariant the $z_{ \pm, r}$ as well as $z_{E}$, namely $a_{\Sigma, i} \rightarrow \epsilon^{\lambda_{\Sigma, i}} a_{\Sigma, i}$ with $\lambda_{\Sigma, i}=c_{\Sigma} \nu_{i, 1}$ parametrized by three constants 
$c_{\Sigma}$ of which $c_{0}$ is irrelevant. The only two classes of inequivalent solutions for the constants $c_{+}, c_{-}$that are compatible with $z_{B} \rightarrow 0$, are $c_{+}^{1}=1, c_{-}^{1}=0$ or $c_{+}^{1}=0, c_{-}^{1}=-1$. The first entry $\nu_{i, 1}$ determines also the $v$ power of the monomial $\prod_{j} x_{j}^{1+\left\langle\nu_{j}^{*}, \nu_{i}\right\rangle}$ multiplying $a_{i}$. So $z_{+, r}$ will be independent of $\epsilon \rightarrow 0$ precisely if $\lambda_{+, i}$ is proportional to the $v$ power of the monomial that multiplies $a_{+, i}$. Therefore the two solutions are precisely the two patches of the local mirror limit in 2.4 , in agreement with our assertion.

For the higher-dimensional case, we can similarly split the vertices $\nu_{i}$ according to the sign of the $n$-th entry $\nu_{i, n}$, whicha gain determines the power of $v$ of the monomial associated to $\nu_{i}$ in the mirror polynomial $p_{\Delta^{*}}$. The rest follows from the above $n=1$ case. 


\section{Appendix B. Topological Data for The Manifolds in Sect.7}

The following tables specify the topological data for the elliptic fibrations above the bases $\tilde{\mathbf{F}}_{k}$ and $\tilde{\mathbf{F}}_{k, m, n}$ described in section 7 . The hodge numbers are given in the first line in the form $\left(h_{\delta h^{1,1}}^{1,1}, h^{1,2}, h_{\delta h^{1,3}}^{1,3}\right)$, where as before $\delta h^{1, i}$ denotes the number of $h^{1, i}$ deformations that are frozen in the toric model. The second line denotes the Euler number $\chi$ and $(\chi \bmod 24)$.

\begin{tabular}{|c|c|c|c|c|c|}
\hline$S U_{1}$ & $S U_{2}$ & $S_{3}$ & $S U_{4}$ & $S U_{5}$ & $S U_{6}$ \\
\hline $\begin{array}{c}\left(11_{0}, 0,30989_{0}\right) \\
186048(0)\end{array}$ & & $k=-18$ & $G^{(2)}=S U_{1}$ & & \\
\hline $\begin{array}{c}\left(12_{0}, 0,28348_{0}\right) \\
170208(0)\end{array}$ & & $k=-17$ & $G^{(2)}=S U_{1}$ & & \\
\hline $\begin{array}{c}\left(12_{0}, 0,25828_{0}\right) \\
155088(0)\end{array}$ & & $k=-16$ & $G^{(2)}=S U_{1}$ & & \\
\hline $\begin{array}{c}\left(12_{0}, 1,23429_{0}\right) \\
140688(0)\end{array}$ & & $k=-15$ & $G^{(2)}=S U_{1}$ & & \\
\hline $\begin{array}{c}\left(12_{0}, 3,21151_{0}\right) \\
127008(0)\end{array}$ & & $k=-14$ & $G^{(2)}=S U_{1}$ & & \\
\hline $\begin{array}{c}\left(12_{0}, 6,18994_{0}\right) \\
114048(0)\end{array}$ & & $k=-13$ & $G^{(2)}=S U_{1}$ & & \\
\hline $\begin{array}{c}\left(12_{0}, 10,16958_{0}\right) \\
101808(0)\end{array}$ & $\begin{array}{c}\left(10_{0}, 0,16959_{0}\right) \\
101862(6)\end{array}$ & $k=-12$ & $G^{(2)}=S U_{1}$ & & \\
\hline $\begin{array}{c}\left(12_{0}, 15,15043_{0}\right) \\
90288(0)\end{array}$ & $\begin{array}{c}\left(10_{0}, 0,15046_{0}\right) \\
90384(0)\end{array}$ & $k=-11$ & $G^{(2)}=S U_{1}$ & & \\
\hline $\begin{array}{c}\left(12_{0}, 21,13249_{0}\right) \\
79488(0)\end{array}$ & $\begin{array}{c}\left(10_{0}, 0,13255_{0}\right) \\
79638(6)\end{array}$ & $k=-10$ & $G^{(2)}=S U_{1}$ & & \\
\hline
\end{tabular}




\begin{tabular}{|c|c|c|c|c|c|}
\hline$S U_{1}$ & $S U_{2}$ & $S U_{3}$ & $S_{4}$ & $S U_{5}$ & $S U_{6}$ \\
\hline & & $k=-9$ & $G^{(2)}=S U_{1}$ & & \\
\hline$\left(12_{0}, 28,11576_{0}\right)$ & $\left(10_{0}, 0,11586_{0}\right)$ & $\left(9_{0}, 0,11587_{0}\right)$ & & & \\
\hline \multirow{2}{*}{$69408(0)$} & $69624(0)$ & $69624(0)$ & & & \\
\hline & & $k=-8$ & $G^{(2)}=S U_{1}$ & & \\
\hline$\left(12_{0}, 36,10024_{0}\right)$ & $\left(10_{0}, 0,10039_{0}\right)$ & $\left(9_{0}, 0,10042_{0}\right)$ & & & \\
\hline \multirow[t]{2}{*}{$60048(0)$} & $60342(6)$ & $60354(18)$ & & & \\
\hline & & $k=-7$ & $G^{(2)}=S U_{1}$ & & \\
\hline$\left(12_{0}, 45,8593_{0}\right)$ & $\left(10_{0}, 0,8614_{0}\right)$ & $\left(9_{0}, 0,8620_{0}\right)$ & & & \\
\hline $51408(0)$ & $51792(0)$ & $51822(6)$ & & & \\
\hline \multirow[b]{2}{*}{$\left(12_{0}, 55,7283_{0}\right)$} & & $k=-6$ & $G^{(2)}=S U_{1}$ & & \\
\hline & $\left(10_{0}, 0,7311_{0}\right)$ & $\left(9_{0}, 0,7321_{0}\right)$ & $\left(8_{0}, 0,7322_{0}\right)$ & & \\
\hline $43488(0)$ & $43974(6)$ & $44028(12)$ & $44028(12)$ & & \\
\hline \multirow[b]{2}{*}{$\left(12_{0}, 66,6094_{0}\right)$} & & $k=-5$ & $G^{(2)}=S U_{1}$ & & \\
\hline & $\left(10_{0}, 0,6130_{0}\right)$ & $\left(9_{0}, 0,6145_{0}\right)$ & $\left(8_{0}, 0,6148_{0}\right)$ & & \\
\hline $36288(0)$ & $36888(0)$ & $36972(12)$ & $36984(0)$ & & \\
\hline \multirow[b]{2}{*}{$\left(12_{0}, 78,5026_{0}\right)$} & & $k=-4$ & $G^{(2)}=S U_{1}$ & & \\
\hline & $\left(10_{0}, 0,5071_{0}\right)$ & $\left(9_{0}, 0,5092_{0}\right)$ & $\left(8_{0}, 0,5098_{0}\right)$ & & \\
\hline $29808(0)$ & $30534(6)$ & $30654(6)$ & $30684(12)$ & & \\
\hline \multirow[b]{2}{*}{$\left(12_{0}, 91,4079_{0}\right)$} & & $k=-3$ & $G^{(2)}=S U_{1}$ & & \\
\hline & $\left(10_{0}, 0,4134_{0}\right)$ & $\left(9_{0}, 0,4162_{0}\right)$ & $\left(8_{0}, 0,4172_{0}\right)$ & $\left(7_{0}, 0,4173_{0}\right)$ & \\
\hline $24048(0)$ & $24912(0)$ & $25074(18)$ & $25128(0)$ & $25128(0)$ & \\
\hline \multirow[b]{2}{*}{$\left(12_{0}, 105,3253_{0}\right)$} & & $k=-2$ & $G^{(2)}=S U_{1}$ & & \\
\hline & $\left(10_{0}, 0,3319_{0}\right)$ & $\left(9_{0}, 0,3355_{0}\right)$ & $\left(8_{0}, 0,3370_{0}\right)$ & $\left(7_{0}, 0,3373_{0}\right)$ & \\
\hline $19008(0)$ & $20022(6)$ & $20232(0)$ & $20316(12)$ & $20328(0)$ & \\
\hline \multirow[b]{2}{*}{$\left(12_{0}, 120,2548_{0}\right)$} & & $k=-1$ & $G^{(2)}=S U_{1}$ & & \\
\hline & $\left(10_{0}, 0,2626_{0}\right)$ & $\left(9_{0}, 0,2671_{0}\right)$ & $\left(8_{0}, 0,2692_{0}\right)$ & $\left(7_{0}, 0,2698_{0}\right)$ & \\
\hline $14688(0)$ & $15864(0)$ & $16128(0)$ & $16248(0)$ & $16278(6)$ & \\
\hline \multirow[b]{2}{*}{$\left(12_{0}, 136,1964_{0}\right)$} & & $k=0$ & $G^{(2)}=S U_{1}$ & & \\
\hline & $\left(10_{0}, 0,2055_{0}\right)$ & $\left(9_{0}, 0,2110_{0}\right)$ & $\left(8_{0}, 0,2138_{0}\right)$ & $\left(7_{0}, 0,2148_{0}\right)$ & $\left(6_{0}, 0,2149_{0}\right)$ \\
\hline $11088(0)$ & $12438(6)$ & $12762(18)$ & $12924(12)$ & $12978(18)$ & $12978(18)$ \\
\hline & & $k=1$ & $G^{(2)}=S U_{1}$ & & \\
\hline$\left(12_{0}, 153,1501_{0}\right)$ & $\left(10_{0}, 0,1606_{0}\right)$ & $\left(9_{0}, 0,1672_{0}\right)$ & $\left(8_{0}, 0,1708_{0}\right)$ & $\left(7_{0}, 0,1723_{0}\right)$ & $\left(6_{0}, 0,1726_{0}\right)$ \\
\hline $8208(0)$ & $9744(0)$ & $10134(6)$ & $10344(0)$ & $10428(12)$ & $10440(0)$ \\
\hline
\end{tabular}




\begin{tabular}{|c|c|c|c|c|c|}
\hline$S U_{1}$ & $S U_{2}$ & $S U_{3}$ & $S U_{4}$ & $S U_{5}$ & $S U_{6}$ \\
\hline & & $k=2$ & $G^{(2)}=S U_{1}$ & & \\
\hline$\left(12_{0}, 171,1159_{0}\right)$ & $\left(10_{0}, 0,1279_{0}\right)$ & $\left(9_{0}, 0,1357_{0}\right)$ & $\left(8_{0}, 0,1402_{0}\right)$ & $\left(7_{0}, 0,1423_{0}\right)$ & $\left(6_{0}, 0,1429_{0}\right)$ \\
\hline \multirow[t]{2}{*}{$6048(0)$} & $7782(6)$ & $8244(12)$ & $8508(12)$ & $8628(12)$ & $8658(18)$ \\
\hline & & $k=3$ & $G^{(2)}=S U_{1}$ & & \\
\hline$\left(12_{0}, 191,939_{0}\right)$ & $\left(10_{0}, 1,1075_{0}\right)$ & $\left(9_{0}, 1,1166_{0}\right)$ & $\left(8_{0}, 1,1221_{0}\right)$ & $\left(7_{0}, 1,1249_{0}\right)$ & $\left(6_{0}, 1,1259_{0}\right)$ \\
\hline \multirow[t]{2}{*}{$4608(0)$} & $6552(0)$ & $7092(12)$ & $7416(0)$ & 7578 (18) & $7632(0)$ \\
\hline & & $k=4$ & $G^{(2)}=S U_{2}$ & & \\
\hline$\left(13_{0}, 210,828_{0}\right)$ & $\left(11_{0}, 0,981_{0}\right)$ & $\left(10_{0}, 0,1086_{0}\right)$ & $\left(9_{0}, 0,1152_{0}\right)$ & $\left(8_{0}, 0,1188_{0}\right)$ & $\left(7_{0}, 0,1203_{0}\right)$ \\
\hline \multirow[t]{2}{*}{$3834(18)$} & $6000(0)$ & $6624(0)$ & $7014(6)$ & $7224(0)$ & $7308(12)$ \\
\hline & & $k=5$ & $G^{(2)}=G_{2}$ & & \\
\hline \multirow{2}{*}{$\begin{array}{c}\left(14_{0}, 231,773_{0}\right) \\
3384(0)\end{array}$} & $\left(12_{0}, 0,944_{0}\right)$ & $\left(11_{0}, 0,1064_{0}\right)$ & $\left(10_{0}, 0,1142_{0}\right)$ & $\left(9_{0}, 0,1187_{0}\right)$ & $\left(8_{0}, 0,1208_{0}\right)$ \\
\hline & $5784(0)$ & $6498(18)$ & $6960(0)$ & $7224(0)$ & $7344(0)$ \\
\hline \multirow{3}{*}{$\begin{array}{c}\left(16_{2}, 253,745_{0}\right) \\
3096(0)\end{array}$} & & $k=6$ & $G^{(2)}=G_{2}$ & & \\
\hline & $\left(14_{2}, 0,935_{0}\right)$ & $\left(13_{2}, 0,1071_{0}\right)$ & $\left(12_{2}, 0,1162_{0}\right)$ & $\left(11_{2}, 0,1217_{0}\right)$ & $\left(10_{2}, 0,1245_{0}\right)$ \\
\hline & $5742(6)$ & $6552(0)$ & $7092(12)$ & $7416(0)$ & $7578(18)$ \\
\hline \multirow{3}{*}{$\begin{array}{c}\left(16_{0}, 276,736_{0}\right) \\
2904(0)\end{array}$} & & $k=7$ & $G^{(2)}=F_{4}$ & & \\
\hline & $\left(14_{0}, 0,946_{0}\right)$ & $\left(13_{0}, 0,1099_{0}\right)$ & $\left(12_{0}, 0,1204_{0}\right)$ & $\left(11_{0}, 0,1270_{0}\right)$ & $\left(10_{0}, 0,1306_{0}\right)$ \\
\hline & $5808(0)$ & $6720(0)$ & $7344(0)$ & $7734(6)$ & $7944(0)$ \\
\hline \multirow{3}{*}{$\begin{array}{c}\left(16_{0}, 300,736_{0}\right) \\
2760(0)\end{array}$} & & $k=8$ & $G^{(2)}=F_{4}$ & & \\
\hline & $\left(14_{0}, 0,967_{0}\right)$ & $\left(13_{0}, 0,1138_{0}\right)$ & $\left(12_{0}, 0,1258_{0}\right)$ & $\left(11_{0}, 0,1336_{0}\right)$ & $\left(10_{0}, 0,1381_{0}\right)$ \\
\hline & $5934(6)$ & $6954(18)$ & $7668(12)$ & $8130(18)$ & $8394(18)$ \\
\hline \multirow{3}{*}{$\begin{array}{c}\left(18_{2}, 325,743_{0}\right) \\
2664(0)\end{array}$} & & $k=9$ & $G^{(2)}=F_{4}$ & & \\
\hline & $\left(16_{2}, 0,996_{0}\right)$ & $\left(15_{2}, 0,1186_{0}\right)$ & $\left(14_{2}, 0,1322_{0}\right)$ & $\left(13_{2}, 0,1413_{0}\right)$ & $\left(12_{2}, 0,1468_{0}\right)$ \\
\hline & $6120(0)$ & $7254(6)$ & $8064(0)$ & $8604(12)$ & $8928(0)$ \\
\hline \multirow{3}{*}{$\begin{array}{c}\left(19_{0}, 351,757_{0}\right) \\
2598(6)\end{array}$} & & $k=10$ & $G^{(2)}=E_{7}$ & & \\
\hline & $\left(17_{0}, 0,1033_{0}\right)$ & $\left(16_{0}, 0,1243_{0}\right)$ & $\left(15_{0}, 0,1396_{0}\right)$ & $\left(14_{0}, 0,1501_{0}\right)$ & $\left(13_{0}, 0,1567_{0}\right)$ \\
\hline & $6348(12)$ & $7602(18)$ & $8514(18)$ & $9138(18)$ & $9528(0)$ \\
\hline \multirow{3}{*}{$\begin{array}{c}\left(19_{0}, 378,775_{0}\right) \\
2544(0) \\
\end{array}$} & & $k=11$ & $G^{(2)}=E_{7}$ & & \\
\hline & $\left(17_{0}, 0,1075_{0}\right)$ & $\left(16_{0}, 0,1306_{0}\right)$ & $\left(15_{0}, 0,1477_{0}\right)$ & $\left(14_{0}, 0,1597_{0}\right)$ & $\left(13_{0}, 0,1675_{0}\right)$ \\
\hline & $6600(0)$ & $7980(12)$ & $9000(0)$ & $9714(18)$ & $10176(0)$ \\
\hline \multirow{3}{*}{$\begin{array}{c}\left(19_{0}, 406,796_{0}\right) \\
2502(6)\end{array}$} & & $k=12$ & $G^{(2)}=E_{7}$ & & \\
\hline & $\left(17_{0}, 0,1121_{0}\right)$ & $\left(16_{0}, 0,1374_{0}\right)$ & $\left(15_{0}, 0,1564_{0}\right)$ & $\left(14_{0}, 0,1700_{0}\right)$ & $\left(13_{0}, 0,1791_{0}\right)$ \\
\hline & $6876(12)$ & $8388(12)$ & $9522(18)$ & $10332(12)$ & $10872(0)$ \\
\hline
\end{tabular}




\begin{tabular}{|c|c|c|c|c|c|}
\hline$S U_{1}$ & $S U_{2}$ & $S U_{3}$ & $S U_{4}$ & $S U_{5}$ & $S U_{6}$ \\
\hline$\left(21_{0}, 441,820_{0}\right)$ & $\left(19_{0}, 6,1171_{0}\right)$ & $\begin{array}{c}k=13 \\
\left(18_{0}, 6,1447_{0}\right)\end{array}$ & $\begin{array}{c}G^{(2)}=E_{8} \times \tilde{S U_{2}} \\
\left(17_{0}, 6,1657_{0}\right)\end{array}$ & $\left(16_{0}, 6,1810_{0}\right)$ & $\left(15_{0}, 6,1915_{0}\right)$ \\
\hline $2448(0)$ & $7152(0)$ & $8802(18)$ & $10056(0)$ & $10968(0)$ & $11592(0)$ \\
\hline$\left(21_{0}, 468,847_{0}\right)$ & $\left(19_{0}, 3,1225_{0}\right)$ & $\begin{array}{c}k=14 \\
\left(18_{0}, 3,1525_{0}\right)\end{array}$ & $\begin{array}{c}G^{(2)}=E_{8} \times \tilde{S U_{2}} \\
\left(17_{0}, 3,1756_{0}\right)\end{array}$ & $\left(16_{0}, 3,1927_{0}\right)$ & $\left(15_{0}, 3,2047_{0}\right)$ \\
\hline $2448(0)$ & $7494(6)$ & $9288(0)$ & $10668(12)$ & $11688(0)$ & $12402(18)$ \\
\hline$\left(21_{0}, 497,876_{0}\right)$ & $\left(19_{0}, 1,1282_{0}\right)$ & $\begin{array}{c}k=15 \\
\left(18_{0}, 1,1607_{0}\right)\end{array}$ & $\begin{array}{c}G^{(2)}=E_{8} \times \tilde{S U} U_{2} \\
\left(17_{0}, 1,1860_{0}\right)\end{array}$ & $\left(16_{0}, 1,2050_{0}\right)$ & $\left(15_{0}, 1,2186_{0}\right)$ \\
\hline $2448(0)$ & $7848(0)$ & $9792(0)$ & $11304(0)$ & $12438(6)$ & $13248(0)$ \\
\hline$\left(21_{0}, 528,907_{0}\right)$ & $\left(19_{0}, 0,1342_{0}\right)$ & $\begin{array}{c}k=16 \\
\left(18_{0}, 0,1693_{0}\right)\end{array}$ & $\begin{array}{c}G^{(2)}=E_{8} \times \tilde{S U_{2}} \\
\left(17_{0}, 0,1969_{0}\right)\end{array}$ & $\left(16_{0}, 0,2179_{0}\right)$ & $\left(15_{0}, 0,2332_{0}\right)$ \\
\hline $2448(0)$ & $8214(6)$ & $10314(18)$ & $11964(12)$ & $13218(18)$ & $14130(18)$ \\
\hline$\left(21_{0}, 561,940_{0}\right)$ & $\left(19_{0}, 0,1405_{0}\right)$ & $\begin{array}{c}k=17 \\
\left(18_{0}, 0,1783_{0}\right)\end{array}$ & $\begin{array}{c}G^{(2)}=E_{8} \times \tilde{S U_{2}} \\
\left(17_{0}, 0,2083_{0}\right)\end{array}$ & $\left(16_{0}, 0,2314_{0}\right)$ & $\left(15_{0}, 0,2485_{0}\right)$ \\
\hline $2448(0)$ & $8592(0)$ & $10854(6)$ & $12648(0)$ & $14028(12)$ & $15048(0)$ \\
\hline$\left(20_{0}, 595,975_{0}\right)$ & $\left(18_{0}, 0,1471_{0}\right)$ & $\begin{array}{c}k=18 \\
\left(17_{0}, 0,1877_{0}\right)\end{array}$ & $\begin{array}{c}G^{(2)}=E_{8} \\
\left(16_{0}, 0,2202_{0}\right)\end{array}$ & $\left(15_{0}, 0,2455_{0}\right)$ & $\left(14_{0}, 0,2645_{0}\right)$ \\
\hline $2448(0)$ & $8982(6)$ & $11412(12)$ & $13356(12)$ & $14868(12)$ & $16002(18)$ \\
\hline
\end{tabular}

Table B.1: Topological data for the elliptic fibrations over $\tilde{\mathbf{F}}_{k}$ dual to $S U(N)$ bundles on elliptic fibrations $Z_{3} \rightarrow \mathbf{P}^{2}$ as described in sect.7.2.

\begin{tabular}{|cccccc|}
\hline$n=7$ & $n=8$ & $n=9$ & $n=10$ & $n=11$ & $n=12$ \\
$\left(51_{0}, 763,1252_{0}\right)$ & $\left(51_{0}, 827,1352_{0}\right)$ & $\left(72_{0}, 886,1454_{0}\right)$ & $\left(72_{0}, 950,1558_{0}\right)$ & $\left(72_{0}, 1014,1662_{0}\right)$ & $\left(73_{1}, 1079,1766_{0}\right)$ \\
$3288(0)$ & $3504(0)$ & $3888(0)$ & $4128(0)$ & $4368(0)$ & $4608(0)$ \\
\hline
\end{tabular}

Table B.2: Topological data for the elliptic fibrations over $\tilde{\mathbf{F}}_{12,12-6 n, n}$ dual to $S U(1)$ bundles on elliptic fibrations $Z_{3} \rightarrow \mathbf{F}_{n}$ as described in sect.7.3. 


\begin{tabular}{|c|c|c|c|c|c|}
\hline$S U_{1}$ & $S U_{2}$ & $S_{3}$ & $S U_{4}$ & $S U_{5}$ & $S U_{6}$ \\
\hline & & $n=-6$ & $G^{(2)}=E_{8}$ & & \\
\hline$\left(41_{0}, 707,1154_{0}\right)$ & $\left(39_{0}, 2,1739_{0}\right)$ & $\left(38_{0}, 2,2212_{0}\right)$ & $\left(37_{0}, 2,2581_{0}\right)$ & $\left(36_{0}, 2,2854_{0}\right)$ & $\left(35_{0}, 2,3039_{0}\right)$ \\
\hline \multirow[t]{2}{*}{$2976(0)$} & $10704(0)$ & $13536(0)$ & $15744(0)$ & $17376(0)$ & $18480(0)$ \\
\hline & & $n=-5$ & $G^{(2)}=E_{8}$ & & \\
\hline$\left(41_{0}, 645,1060_{0}\right)$ & $\left(39_{0}, 0,1593_{0}\right)$ & $\left(38_{0}, 0,2022_{0}\right)$ & $\left(37_{0}, 0,2355_{0}\right)$ & $\left(36_{0}, 0,2600_{0}\right)$ & $\left(35_{0}, 0,2765_{0}\right)$ \\
\hline \multirow[t]{2}{*}{$2784(0)$} & $9840(0)$ & $12408(0)$ & $14400(0)$ & $15864(0)$ & $16848(0)$ \\
\hline & & $n=-4$ & $G^{(2)}=E_{8}$ & & \\
\hline$\left(31_{0}, 597,974_{0}\right)$ & $\left(28_{0}, 2,1470_{0}\right)$ & $\left(28_{0}, 2,1872_{0}\right)$ & $\left(27_{0}, 2,2191_{0}\right)$ & $\left(26_{0}, 2,2434_{0}\right)$ & $\left(25_{0}, 2,2609_{0}\right)$ \\
\hline \multirow[t]{2}{*}{$2496(0)$} & $9024(0)$ & $11436(12)$ & $13344(0)$ & $14796(12)$ & $15840(0)$ \\
\hline & & $n=-3$ & $G^{(2)}=E_{8}$ & & \\
\hline$\left(26_{0}, 552,902_{0}\right)$ & $\left(24_{0}, 1,1361_{0}\right)$ & $\left(23_{0}, 1,1736_{0}\right)$ & $\left(22_{0}, 1,2035_{0}\right)$ & $\left(21_{0}, 1,2266_{0}\right)$ & $\left(20_{0}, 1,2437_{0}\right)$ \\
\hline \multirow[t]{2}{*}{$2304(0)$} & $8352(0)$ & $10596(12)$ & $12384(0)$ & $13764(12)$ & $14784(0)$ \\
\hline & & $n=-2$ & $G^{(2)}=E_{8}$ & & \\
\hline$\left(21_{0}, 529,868_{1}\right)$ & $\left(19_{0}, 0,1309_{1}\right)$ & $\left(18_{0}, 0,1670_{1}\right)$ & $\left(17_{0}, 0,1959_{1}\right)$ & $\left(16_{0}, 0,2184_{1}\right)$ & $\left(15_{0}, 0,2353_{1}\right)$ \\
\hline \multirow[t]{2}{*}{$2208(0)$} & $8016(0)$ & $10176(0)$ & $11904(0)$ & $13248(0)$ & $14256(0)$ \\
\hline & & $n=-1$ & $G^{(2)}=E_{8}$ & & \\
\hline \multirow{2}{*}{$\begin{array}{c}\left(21_{0}, 529,868_{0}\right) \\
2208(0)\end{array}$} & $\left(19_{0}, 0,1309_{0}\right)$ & $\left(18_{0}, 0,1670_{0}\right)$ & $\left(17_{0}, 0,1959_{0}\right)$ & $\left(16_{0}, 0,2184_{0}\right)$ & $\left(15_{0}, 0,2353_{0}\right)$ \\
\hline & $8016(0)$ & $10176(0)$ & $11904(0)$ & $13248(0)$ & $14256(0)$ \\
\hline \multirow{3}{*}{$\begin{array}{c}\left(21_{0}, 529,868_{0}\right) \\
2208(0)\end{array}$} & & $n=0$ & $G^{(2)}=E_{8}$ & & \\
\hline & $\left(19_{0}, 0,1309_{0}\right)$ & $\left(18_{0}, 0,1670_{0}\right)$ & $\left(17_{0}, 0,1959_{0}\right)$ & $\left(16_{0}, 0,2184_{0}\right)$ & $\left(15_{0}, 0,2353_{0}\right)$ \\
\hline & $8016(0)$ & $10176(0)$ & $11904(0)$ & $13248(0)$ & $14256(0)$ \\
\hline
\end{tabular}

Table B.3: Topological data for the elliptic fibrations over $\tilde{\mathbf{F}}_{12,12-6 n, n}$ dual to $S U(N)$ bundles on elliptic fibrations $Z_{3} \rightarrow \mathbf{F}_{n}$ as described in sect.7.3. 


\section{References}

[1] P. Candelas, G. Horowitz, A. Strominger and E. Witten, Nucl. Phys. $\underline{B 258}$ (1985) 46.

[2] E. Witten, Nucl. Phys. $\underline{B 268}$ (1986) 79.

[3] C. Vafa, Nucl. Phys. $\underline{B 469}$ (1996) 403.

[4] A. Sen, Nucl. Phys. B475 (1996) 562.

[5] C. Vafa and D. Morrison, Nucl. Phys. $\underline{B 473}$ (1996) 74;Nucl. Phys. $\underline{B 476}$ (1996) 437.

[6] J. Distler, B.R. Greene and D.R. Morrison, Nucl. Phys. B481 (1996) 289.

[7] E. Looijenga, Invent. Math. $\underline{38}$ (1977) 17; Invent. Math. $\underline{61}$ (1980) 1.

[8] R. Friedman, J.W. Morgan and E. Witten, Comm. Math. Phys. $\underline{187}$ (1997) 679 .

[9] R. Friedman, J.W. Morgan and E. Witten, Principal G-bundles over elliptic curves, alg-geom/9707004; Vector Bundles over Elliptic Fibrations, alg-geom/9709029.

[10] M. Bershadsky, A. Johansen, T. Pantev and V. Sadov, Nucl. Phys. $\underline{B 505}$ (1997) 165.

[11] S. Katz, P. Mayr and C. Vafa, Adv. Theor. Math. Phys. 1 (1998) 53.

[12] C. Vafa and E. Witten, Nucl. Phys. Proc. Suppl. 46 (1996) 225.

[13] N. Seiberg and E. Witten, Nucl. Phys. $\underline{B 426}$ (1994) 19, erratum: ibid $\underline{430}$ (1994) 396; Nucl. Phys. Bu31 (1994) 484.

[14] S. Kachru, A. Klemm, W. Lerche, P. Mayr and C. Vafa, Nucl. Phys. $\underline{B 459}$ (1996) 537.

[15] A. Klemm, W. Lerche, P. Mayr, C. Vafa, N. Warner, Nucl. Phys. $\underline{B 477}$ (1996) 746.

[16] S. Katz, A. Klemm and C. Vafa, Nucl. Phys. B497 (1997) 173.

[17] R.S. Aspinwall, B.R. Greene and D.R. Morrison, Nucl. Phys. $\underline{B} 416$ (1994) 414; P. Candelas, X. de la Ossa and S. Katz, Nucl. Phys. $\underline{B 450}$ (1996) 267; S. Katz, P. Mayr and C. Vafa as quoted in [11] ; N.C. Leung and C. Vafa, Adv. Theor. Math. Phys. $\underline{2}$ (1998) 91. 
[18] V. Batyrev, Duke Math. Journ. $\underline{69}$ (1993) 349.

[19] M. Bershadsky et al., Nucl. Phys. B481 (1996) 215.

[20] P. Candelas and A. Font, Nucl. Phys. B511 (1998) 295.

[21] H. Pinkham, C. R. Acad. Sci. Paris $\underline{284 A}$ (1977) 615;

I.V. Dolgachev, J. Math. Sciences $\underline{81}$ (1996) 2599.

[22] A. C. Avram, M. Kreuzer, M. Mandelberg and H. Skarke, Nucl. Phys. $\underline{B 494}$ (1997) 567.

[23] S. Katz, P. Mayr and C. Vafa, unpublished

[24] P.S. Aspinwall, Nucl. Phys. $\underline{B 496}$ (1997) 149; P.S. Aspinwall and D.R. Morrison, Nucl. Phys. B B503 (1997) 533.

[25] P.S. Aspinwall, J. High Energy Phys. $\underline{4}$ (1998) 19.

[26] P.S. Aspinwall and R.Y. Donagi, The Heterotic string, the tangent bundle, and derived categories, hep-th/9806094.

[27] M. Kreuzer and H. Skarke, J. Geom. Phys. 26 (1998) 272.

[28] S. Katz, D.R. Morrison and M.R. Plesser, Nucl. Phys. B477 (1996) 105.

[29] M.J. Duff, R. Minasian and E. Witten, Nucl. Phys. $\underline{B 465}$ (1996) 413.

[30] M. Berkooz et al., Nucl. Phys. B475 (1996) 115.

[31] A. Sen, Nucl. Phys. $\underline{B 498}$ (1997) 135.

[32] P. Berglund, S. Katz, A. Klemm, and P. Mayr, to appear.

[33] P. Candelas and H. Skarke, Phys. Lett. B413 (1997) 63.

[34] E. Witten, Nucl. Phys. $\underline{B 460}$ (1996) 541.

[35] P. Mayr, Geometric construction of $N=2$ gauge theories, hepth/9807096.

[36] P. Candelas, E. Perevalov and G. Rajesh, Nucl. Phys. $\underline{B 507}$ (1997) 445.

[37] P. Ginsparg, Phys. Rev. D35 (1987) 648.

[38] J.D. Blum and K. Intriligator, Nucl. Phys. B506 (1997) 199; Nucl. Phys. $\underline{B 506}$ (1997) 223.

[39] S. Katz and C. Vafa, Nucl. Phys. $\underline{B 497}$ (1997) 146. 
[40] K. Intriligator and N. Seiberg, Phys. Lett. B387 (1996) 513;

J. de Boer, K. Hori, H. Ooguri and Y. Oz, Nucl. Phys. B493 (1997) 101;

M. Porrati and A. Zaffaroni, Nucl. Phys. $\underline{B} 490$ (1997) 107;

K. Hori, H. Ooguri and C. Vafa, Nucl. Phys. B504 (1997) 147.

[41] E. Perevalov and G. Rajesh, Phys. Rev. Lett. $\underline{79}$ (1997) 2931.

[42] S. Chaudhuri, G. Hockney and J.D. Lykken, Phys. Rev. Lett. $\underline{75}$ (1995) 2264 .

[43] P. Berglund, A. Klemm, P. Mayr and S. Theisen, On type IIB vacua with varying coupling constant, hep-th/9805189.

[44] A. Klemm, W. Lerche and P. Mayr, Phys. Lett. $\underline{B}$ (1995) 313.

[45] S. Kachru and E. Silverstein, Nucl. Phys. B482 (1996) 92.

[46] P. Mayr, Nucl. Phys. B4494 (1997) 489.

[47] A. Klemm, B. Lian, S.S. Roan and S.T. Yau, Nucl. Phys. B518 (1998) 515 .

[48] K. Mohri, $F$ theory vacua in four-dimensions and toric threefolds, hepth/9701147.

[49] M. Kreuzer and H. Skarke, J. Geom. Phys. $\underline{26}$ (1998) 272.

[50] M.R. Douglas, Gauge fields and D-branes, hep-th 9604198.

[51] S. Sethi, C. Vafa and E. Witten, Nucl. Phys. B4480 (1996) 213.

[52] K. Becker and M. Becker, Nucl. Phys. B477 (1996) 155.

[53] M. Green, J.A. Harvey and G. Moore, Class. Quant. Grav. 14 (1997) 47

[54] P. Berglund and S. Theisen, unpublished.

[55] B. Andreas, G. Curio and D. Lüst, Nucl. Phys. B507 (1997) 175.

[56] B. Andreas, On vector bundles and chiral matter in $N=1$ heterotic compactifications, hep-th/9802202; G. Curio, Phys. Lett. $\underline{B 435}$ (1998) 39.

[57] V.V. Batyrev, J. Alg. Geom. $\underline{3}$ (1994) 493; V.V. Batyrev, D.I. Dais, Topology $\underline{35}$ (1996) 901; V.V. Batyrev and L.A. Borisov, Invent. Math. $\underline{126}$ (1996) 183.

[58] E. Witten, Comm. Math. Phys. 117 (1988) 353; Mirror manifolds and topological field theory. In: Essays on Mirror Manifolds (ed. S.-T. Yau), Hong Kong: Int. Press, 1992, pp. 120-158. 
[59] B.R. Greene, D.R. Morrison and M.R. Plesser, Comm. Math. Phys. $\underline{173}$ (1995) 559.

[60] W. Lerche, J. High Energy Phys. 11 (1997) 4; Nucl. Phys. Proc. Suppl. 68(1998) 252.

[61] W. Lerche and S. Stieberger, Prepotential, mirror map and $F$ theory on K3, hep-th/ 9804176.

[62] E. Witten, Nucl. Phys. $\underline{B 474}$ (1996) 343.

[63] S. Katz and C. Vafa, Nucl. Phys. $\underline{B 497}$ (1997) 204;

C. Vafa, Adv.Theor.Math.Phys. 2 (1998) 497.

[64] G. Curio and R.Y. Donagi, Nucl. Phys. $\underline{B 518}$ (1998) 603.

[65] G. Curio, Phys. Lett. $\underline{B 435}$ (1998) 39.

[66] P. Aspinwall, Phys. Lett. $\underline{B 357}$ (1995) 329. 